This item was submitted to Loughborough's Research Repository by the author.

Items in Figshare are protected by copyright, with all rights reserved, unless otherwise indicated.

\title{
Stereo-PIV measurements of spatio-temporal turbulence correlations in an axisymmetric jet
}

\section{PLEASE CITE THE PUBLISHED VERSION}

http://dx.doi.org/10.1017/jfm.2015.362

\section{PUBLISHER}

(c) Cambridge University Press

\section{VERSION}

AM (Accepted Manuscript)

\section{PUBLISHER STATEMENT}

This work is made available according to the conditions of the Creative Commons Attribution-NonCommercialNoDerivatives 4.0 International (CC BY-NC-ND 4.0) licence. Full details of this licence are available at: https://creativecommons.org/licenses/by-nc-nd/4.0/

\section{LICENCE}

CC BY-NC-ND 4.0

\section{REPOSITORY RECORD}

Pokora, C.D., and Jim McGuirk. 2015. "Stereo-piv Measurements of Spatio-temporal Turbulence Correlations in an Axisymmetric Jet". Loughborough University. https://hdl.handle.net/2134/18474. 


\title{
Stereo-PIV Measurements of Spatio-Temporal Turbulence Correlations in an Axisymmetric Jet
}

\author{
C. D. POKORA ${ }^{1}$ AND J. J. MCGUIRK \\ Dept. of Aeronautical and Automotive Engineering, \\ Loughborough University, Leicestershire, LE11 3TU, UK
}

Stereoscopic 3-component Particle Image Velocimetry (3C-PIV) measurements have been made in a turbulent round jet to investigate the spatio-temporal correlations that are the origin of aerodynamic noise. Restricting attention to subsonic, isothermal jets, measurements were taken in a water flow experiment where, for the same Reynolds number and nozzle size, the shortest time scale range of the dynamically important turbulent structures is more than an order of magnitude greater that in equivalent airflow experiments, greatly facilitating time-resolved PIV measurements. Results obtained (for a jet nozzle diameter/velocity of $40 \mathrm{~mm}$ and $1 \mathrm{~m} / \mathrm{s}$, giving $R e=4 \times 10^{4}$ ) show that, on the basis of both 1-point statistics and 2-point quantities (correlation functions, integral length scales) the current incompressible flow data are in excellent agreement with published compressible, subsonic airflow measurements. The 3C-PIV data are first compared to higher spatial resolution 2C-PIV data and observed to be in good agreement, although some deterioration in quality for higher-order correlations caused by high frequency noise in the 3C-PIV data is noted. A filter method to correct for this is proposed, based on Proper Orthogonal Decomposition of the 3C-PIV data. The corrected data are then used to construct correlation maps at $2^{\text {nd }}$ and $4^{\text {th }}$-order level for all velocity components. The present data are in accordance with existing hot-wire measurements, but provide significantly more detailed information on correlation components than has previously been available. The measured relative magnitudes of various components of the 2-point $4^{\text {th }}$-order turbulence correlation coefficient $\left(R_{i j, k l}\right)$ - the fundamental building block for free shear flow aerodynamic noise sources - are presented and represent a valuable source of validation data for acoustic source modelling. The relationship between $4^{\text {th }}$ order and $2^{\text {nd }}$-order velocity correlations is also examined, based on an assumption of a quasi-Gaussian nearly normal pdf for the velocity fluctuations. The present results indicate that this approximation shows reasonable agreement for the measured relative magnitudes of several correlation components; however, areas of discrepancy are identified, indicating the need for work on alternative models such as the shell turbulence concept of Afsar (2012).

\section{Introduction}

For some considerable time, one of the most important areas of research in jet acoustics has been the development and application of improved techniques for accurate measurement of whole-field spatio-temporal turbulence correlations. The $4^{\text {th }}$-order 2point 2-time correlation of the fluctuating Reynolds stress ( $r_{i j, k l}$ - to be defined below) has long been recognised as the fundamental statistical property of jet turbulence, acting as the acoustic source in broadband jet mixing noise (Lighthill (1952), (1954)). Proudman (1952), for example, used the Lighthill theory to show that the acoustic intensity at any

${ }^{1}$ Current address: Tata Technologies, Bristol BS16 1EJ, UK. 


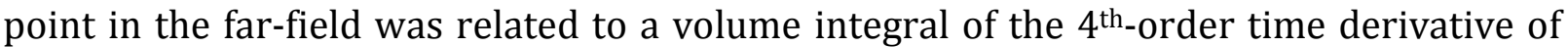
the 2-point 2-time correlation of the fluctuating Reynolds stress tensor. Assumptions about this property are an inherent part of all jet noise models (Morris and Farassat (2002)). Direct measurements of this correlation to judge the accuracy of the various approximations proposed for its modelling are therefore crucial for progress towards improved jet noise simulation.

Historically, data on spatio-temporal correlations in jets were first obtained using hotwire anemometry (HWA). This has excellent temporal response - up to $\sim 50 \mathrm{kHz}$ - but it is a single point technique and spatial resolution relies on traverse of multiple HWA probes with respect to each other. The first HWA measurements of spatial turbulence correlations in jets were reported by Laurence (1956), although at this early stage of electronic signal processing only for the $2^{\text {nd }}$ order correlation $\left(r_{i j}\right)$ and its correlation coefficient $\left(R_{i j}\right.$ - the auto-covariance of the fluctuating velocity $u_{i}^{\prime}$ ):

$$
r_{i j}(\underline{x}, \underline{\eta}, \tau)=\overline{u_{i}^{\prime}(\underline{x}, t) u_{j}^{\prime}(\underline{x}+\underline{\eta}, t+\tau)} \quad R_{i j}(\underline{x}, \underline{\eta}, \tau)=\frac{r_{i j}(\underline{x}, \underline{\underline{\eta}}, \tau)}{\sqrt{\overline{u_{i}^{\prime 2}}}(\underline{x}) \sqrt{\overline{u_{j}^{\prime 2}}}(\underline{x}+\underline{\eta})}
$$

Here an overbar indicates the usual Reynolds time-averaging, with $\underline{\eta}$ and $\tau$ the spatial and temporal separations respectively. Instrumentation limitations restricted the data set in Laurence (1956) to zero time delay and axial separation; information on integral length and time scales were extracted from the longitudinal and radial velocity correlation functions. Using band-pass filters applied to the HWA signals, the concept of frequencydependant integral scales was also introduced - this has been re-visited recently by Harper-Bourne (1993), (2003) and Kerherve et al. (2006) as an aid to acoustic source modelling. The Laurence data were also used by Lilley (1958) as input into one of the first computational models for jet noise.

The HWA measurements most often used to support jet acoustics modelling were carried out by Davies et al. (1962) and Fisher and Davies (1964). The first paper produced clear evidence of similarity of turbulence intensity, length/time scales, and energy spectra. The rate of decay in the peak value of the measured spatial correlations with increasing downstream separation was used to deduce a turbulent eddy convection velocity, which was found not to be equal to the local mean velocity, but rather $\sim 0.6$ times the jet velocity and varying slightly over the maximum shear region - this concept has been used in many acoustic source models ever since. It was also noted that this factor varied if the spectral content of turbulence was considered; length scales associated within different frequency bands were observed to travel at different speeds, an observation later confirmed by Harper-Bourne (2003), and made use of in the recently proposed modification to the Lighthill acoustic analogy of Self (2004). The most comprehensive early HWA investigation into 2-point quantities was that of Bradshaw et al. (1964). $2^{\text {nd }- \text { order }}\left(R_{i j}\right)$ data were reported for all 3 velocity components and with respect to all 3 separation directions. Interestingly, the dominance of the large eddies in establishing correlation shapes and convection velocity lead to the first speculation in this paper that "augmentation of large eddies by artificial devices could be used to increase mixing and permit reduction of jet noise" - an idea taken up in the last decade or so via use of jet nozzles with serrated trailing edges (Alkislar et al. (2007)). The paper also contains the statement that "measurement of the fluctuating stress tensor, which is the forcing term in Lighthill's equation for noise emissions, seems out of the question".

In spite of this statement (although 40 years later) Harper-Bourne (2003) and Morris and Zaman (2010) were successful in extending the HWA technique to measurements of 
both $2^{\text {nd }}$ - and $4^{\text {th }}$-order correlations in round jets and providing a data set that included streamwise, radial, and circumferential separations, although only for the streamwise velocity component. Morris and Zaman (2010) described how various definitions may be

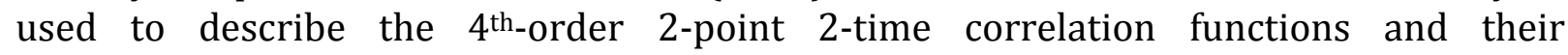
corresponding correlation coefficients. The form used here for the correlation function is:

$$
r_{i j, k l}(\underline{x}, \underline{\eta}, \tau)=\overline{\left[u_{i}^{\prime} u_{j}^{\prime}-\overline{u_{i}^{\prime} u_{j}^{\prime}}\right](\underline{x}, t)\left[u_{k}^{\prime} u_{l}^{\prime}-\overline{\left.u_{k}^{\prime} u_{l}^{\prime}\right]}(\underline{x}+\underline{\eta}, t+\tau)\right.}
$$

(this is the form chosen by Karabasov et al. (2010) to suit the particular acoustic analogy model adopted). Similarly, various routes have been suggested (Morris and Zaman (2010)) for the normalisation factor used to form the corresponding correlation coefficient $\left(R_{i j, k l}\right)$; the precise choice is not important as long as the coefficient is nondimensional. The approach used here is taken from Pokora and McGuirk (2008) and Pokora (2009), who were primarily interested in the relative magnitudes of the peak values of the various components of $R_{i j, k l_{;}}$- this approach makes components $R_{\alpha \alpha, \alpha \alpha}$ (no summation on $\alpha$ ) equal to unity at zero space/time separations and forms a reasonable basis for comparison:

$$
R_{i j, k l}(\underline{x}, \underline{\eta}, \tau)=\frac{r_{i j, k l}}{\left[\overline{u_{i}^{\prime 4}}(A) \overline{u_{j}^{\prime 4}}(A) \overline{u_{k}^{\prime 4}}(B) \overline{u_{l}^{\prime 4}}(B)\right]^{1 / 4}-\left[\overline{u_{i}^{\prime 2}}(A) \overline{u_{j}^{\prime 2}}(A) \overline{u_{k}^{\prime 2}}(B) \overline{u_{l}^{\prime 2}}(B)\right]^{1 / 2}}
$$

where: $A=(\underline{x}), B=(\underline{x}+\underline{\eta})$.

The main motivation of the Harper-Bourne (2003) study was the derivation of a Lighthill acoustic analogy model based on a fixed frame of reference (rather than the moving frame normally adopted, hence the introduction of the eddy convection velocity). It was shown how this approach enabled a more deterministic link between jet turbulence statistics measurements and models for far-field noise prediction, and such models have since been proposed, e.g. Leib and Goldstein (2011). Morris and Zaman (2010) used both single wire and cross-wire probes to provide $2^{\text {nd }}$ and $4^{\text {th }}$-order correlations with respect to streamwise separations for both streamwise and radial velocity components. Their measurements for $r_{i j}$ and $r_{i j, k l}$ were also used to examine a relationship between $2^{\text {nd }}$ - and $4^{\text {th }}$-order correlations, following a suggestion of Lighthill; the motivation was to support the development of more practical noise source models which depend on $2^{\text {nd }}$-order quantities only. Comparison with the experimental data proved successful, although with only limited validity, and was only applicable for components of the $4^{\text {th }}$-order tensor with all indices equal.

The intrusive nature of HWA probe-based single-point measurements inevitably meant that extracting data on all components of $r_{i j, k l}$ was problematic and hence only limited data has been produced. Attention was turned to use of non-intrusive laser-based methods when these became available. Laser Doppler Anemometry (LDA) - as for HWA probes applied in traversed multi-point mode - was successfully used by Lau et al. (1979) and Lau (1980) for $2^{\text {nd }}$-order correlations, and by Kerherve et al. (2004) to study the longitudinal component of both $2^{\text {nd }}$ - and $4^{\text {th }}$-order correlations, for axial separation only, in a high speed shear layer, enabling analytical expressions to be derived for the spatial and temporal parts of the correlation function. However, the point-based nature of LDA still seriously constrains its use in extraction of whole-field spatio-temporal correlation maps, in principle needed for all three velocity components and for all components of the separation vector. Initial attempts to combine single-point and spatial (planar) laser- 
based techniques (e.g. LDA with Particle Image Velocimetry (PIV)) by Chatellier and Fitzpatrick (2005) - to combine the convenient spatial resolution offered by PIV with the better temporal resolution of LDA - were only partially successful. No convincing demonstration that this approach could produce correlations competitive in accuracy with existing HWA measurements has emerged.

In contrast, the continued improvement of hardware and software components of the PIV technique in recent years means this has become the measurement technique of choice for correlation mapping. This approach is, however, also not without its problems. The need to avoid spatial filtering of turbulence, when using planar PIV with a large Field of View (FoV) relative to the local integral scales of turbulence, is well known. HoestMadsen \& Nielson (1995) showed that significant loss of accuracy in $2^{\text {nd }}$-order 1-point turbulence statistics results if the PIV interrogation window/cell is not small in relation to the local integral scale. If careful attention is paid to matching the FoV size to local flow characteristics then this problem can be avoided. An example of this was provided by Midgley et al. (2005), who adopted a hybrid FoV approach. The FoV size was chosen to suit the local (measured) turbulent flow structures, so that complete flowfield maps were patched together using a range of appropriate FoV sizes. This required an iterative approach whereby data taken on initial large FoVs (and the integral scales evaluated from these via spatial integration of measured $r_{i j}$ functions) were used to identify thin shear layer flow regions where the large FoV interrogation cells caused sub-cell PIV loss of turbulence, and measurements on smaller FoVs were then carried out. This approach is not in general compatible when measurement of correlation maps with large 2-point separation values - of order $>1$ jet diameter for example - are of interest. Equally, up to 3 layers of embedded FoVs were required and the method is therefore time-consuming. Correction factors for the sub-cell filtering may be introduced to address this problem, as demonstrated by Spencer and Hollis (2005) based on an extension of the Hoest Madsen and Nielson (1995) concept. Alkislar (2007) used an alternative approach based on consistency with the RANS mean momentum equations and the measured mean velocity data (which do not suffer from the spatial filtering effect). These studies have shown that the loss of accuracy even in single point $2^{\text {nd }}$-order statistics due to spatial filtering can be as large as $30 \%$ when the PIV cell size is of the same order as the local integral scale. To reduce the error to less than $10 \%$, a PIV interrogation cell size of order $1 / 10$ th of the integral scale is needed. Alkislar (2005) indicated $100 \%$ error can occur in PIV measurements of turbulent normal stresses in the first diameter of flow development on the lip-line of a nozzle, so this problem cannot be ignored. It will undoubtedly be significantly more serious for higher order statistics, so careful checks on adequate spatial resolution is a crucial aspect for any PIV study aimed at producing data for guidance of models for $r_{i j}$ and $r_{i j, k l}$, although rarely carried out in measurements published to date.

The problem of measuring correlation maps for jet noise application with large values of the separation vector, but avoiding the problem of too large FoV's, was addressed by work at NASA reported in Bridges and Wernet (2003) by using a dual camera planar PIV system for two subsonic jet Mach numbers (0.5 and 0.9) and both cold and heated jets. Measurements from each laser system were time-co-ordinated and 2000x2000 pixel dual-frame cameras were used viewing the light sheet at right angles, and thus measuring axial and radial velocity components only on a plane containing the jet axis. The FoV was $170 \mathrm{~mm}$ square, with a jet nozzle of exit diameter $D_{j}=50.1 \mathrm{~mm}$; final data maps thus had a spatial resolution of $\sim 1 \mathrm{~mm}$ or $0.02 D_{j}$, and time-averages were collected over 200 frames. A cross-polarisation technique (see Bridges and Wernet (2003)) was included to avoid 
light from one PIV system acting as noise on the $2^{\text {nd }}$ system. Difficulties were experienced in obtaining unity values of the correlations at zero space/time separations due to the problem of arranging perfect alignment of two imaging systems. Perhaps because of this, although direct measurements of $r_{11,11}$ and $r_{22,22}$ were presented, these displayed high levels of noise due to spatial filtering problems and no direct comparisons were made between the $2^{\text {nd }}$ - and $4^{\text {th }}$-order PIV-measured correlations and previous HWA or other

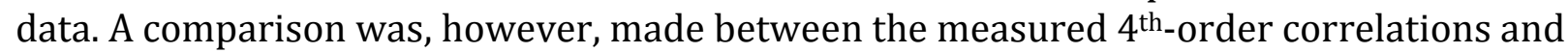
approximations of these via an expression involving products of $2^{\text {nd }}$-order correlations based on an assumption of normal joint probability distributions for velocity. It was concluded that reasonable agreement was seen in spite of large uncertainties in the $4^{\text {th }}$ order data. A similar study using dual-PIV measurements has been reported by Fleury et al. (2008), again for two subsonic Mach numbers (0.5 and 0.9). FoV size in this work was $84 \mathrm{~mm} \times 64 \mathrm{~mm}$, with PIV camera resolution of $1280 \times 1024$ pixels; spatial resolution was thus similar to Bridges and Wernet (2003)). This work concentrated on $2^{\text {nd }}$-order 2-point correlation measurements. No attempt was made to provide direct correlation comparison against HWA data, but integral length and timescales extracted from measured $r_{i j}$ were in good agreement with HWA measurements. Note that for single point turbulence statistics as well as integral scales, no effect of Mach number was found comparing $\mathrm{M}=0.5$ and $=0.9$ data.

The NASA work described above was recently extended to improve the temporal resolution of the dual-PIV technique (Wernet (2007)). For airflows at high jet Reynolds and Mach numbers, the temporal resolution capability of PIV systems is severely challenged by the required high data rate to capture the energy-containing fluctuation frequency range of interest; this is typically up to $20 \mathrm{kHz}$, and even higher for hot jets. The rapid development of laser and camera technology has, however, meant that progress has been made towards increasing maximum PIV temporal resolution. Using special high speed cameras, Wernet (2007) has presented data taken at planar PIV double frame rates of $10 \mathrm{kHz}$ and $25 \mathrm{kHz}$ for time durations of $\sim 2$ seconds. The results presented indicated that $10 \mathrm{kHz}$ was a realistic upper bound for accurate measurement, because of the reduction in size of the data acquisition window necessary to achieve the higher frame rate. Most effort was devoted to assessment of the spectral accuracy of the new TR (Time-Resolved) PIV system, and development of special techniques (e.g. anti-aliasing filters) to produce spectra in good agreement with HWA. Further application of the technique has mainly focussed on identification of a 'consensus' data set for (mainly 1point) turbulence statistics for higher Mach number hot jets (Bridges \& Wernet (2010), (2012)), no further attempts to measure $4^{\text {th }}$-order correlations have been reported.

Based on the above review the present work was aimed at producing direct measurements of $r_{i j, k l}$ in a representative jet flow. It is important to consider what exactly is meant by 'representative'. The engineering problem behind the present research has been defined as jet acoustics, and this seems naturally to imply that compressibility must be important and included in any relevant experimental investigation. However, if attention is focussed on the turbulent correlations that are the source of far-field noise rather than the noise itself, this is not necessarily so. In a free jet the turbulent correlations of interest are created by the shear between the jet flow and the stagnant ambient. If the jet is subsonic, then even if the jet Mach number $\left(\mathrm{M}_{\mathrm{J}}\right)$ is high to influence the mean flow, it is known that compressibility effects do not always play a dominant role on the turbulence. The experiments of Papamoschou and Roshko (1988) (and many others) have established that only when the convective Mach number $\left(\mathrm{M}_{\mathrm{C}}\right)$ is high (e.g. 
$M_{C}>0.6$ ) does the spreading rate of a turbulent shear layer display any evidence of compressibility effects. $M_{C}$ is the Mach number in a frame of reference moving with the speed of the instability waves (or other disturbances such as turbulent structures) in the shear layer; for a subsonic jet in stagnant surroundings $\mathrm{M}_{\mathrm{J}} \sim 0.5 \mathrm{M}_{\mathrm{J}}$. Thus, for subsonic jet Mach numbers this implies compressibility effects will not be influential in determining the turbulence correlations of interest. This is borne out on examination of experiments which have matched jet Mach numbers - for example the data of Fleury et al. 2008 which showed no evidence of jet Mach number effects when this was changed from 0.6 to 0.9. If it not important to match Mach number, for unheated jets this just leaves the question of matching Reynolds number. Here also there is ample evidence of similarity behaviour at different jet velocities as long as the jet Reynolds number is kept sufficiently high (further details given in Section 2). It follows from these considerations that, if neither Mach number nor Reynolds number matching is crucial, an experiment can still be considered representative if a liquid flow rather than an air flow experiment were selected, as long as a sufficiently high Reynolds number is ensured. For water flow for example, for the same nozzle size and Reynolds number, this lowers the frequency range of the dynamically active turbulence structures of interest by a factor of $\sim 15$ and shifts this into the range of $\sim 1 \mathrm{kHz}$ available from currently available low cost commercial PIV systems. Mindful of the emphasis given above of the importance of adequate spatial and temporal resolution, a water flow experiment thus offers clear advantages. It is important to understand the limitations of this approach. The data obtained will not be relevant to high speed (supersonic) jet flows which may contain regions of under- or over-expansion, or where the jet/ambient convective Mach number is sufficiently high to cause the well-known compressibility-caused reduction in shear layer spreading rate; similarly, the influence on the turbulent correlations of a heated jet cannot be assessed using the approach taken here. However, given the lack of accurate data on $4^{\text {th }}$-order correlations noted above, if such data could be obtained in this way it would still be important as validation data for aeroacoustic modelling of subsonic isothermal turbulent jets.

In what follows it is first demonstrated that the measured turbulence structure in such an experiment is still clearly representative of high subsonic jet shear layers. Two component (2C) measurements in a carefully designed water flow experiment are first compared with previous HWA data and dual camera PIV data taken in airflow experiments for correlation coefficients and extracted qualities such as integral length scales and shown to be of comparable accuracy. The method of Spencer and Hollis (2005) is used to monitor and assess spatial filtering effects. The opportunity is then taken to use stereo (three component (3C)) PIV to gather correlation data for many more components of $r_{i j, k l}$ than has previously been possible. Because of the opposing demands of small FoV for accuracy but large FoV for large separation vectors in the correlations, it was found that a correction procedure was required for the 3C-PIV data; this is based on a POD analysis and is fully described below.

\section{Experimental Methodology}

\subsection{Experimental Facility}

The experiments were performed in a water tunnel facility used in previous jet flow experimental research (Behrouzi and McGuirk (2004), (2006)), see figure 1(a). The tunnel is of re-circulating design and can accommodate both single and co-axial nozzles. The test section is $2010 \mathrm{~mm}$ long, $375 \mathrm{~mm}$ wide and 300 high. Perspex side walls as well as 
roof/ceiling enable non-intrusive measurement techniques to be used to measure the flow. The flow was seeded with neutrally buoyant polyamide seeding particles with a mean particle diameter of $20 \mu \mathrm{m}$. Due to the recirculating design of the tunnel complete seeding saturation was possible; uniform seeding density was achieved in both jet and surrounding tunnel flow, $\sim 10$ particles per PIV interrogation window. An upstream contraction and turbulence management screens ensured good uniformity of any coflowing stream outside the jet; preliminary measurements showed this was uniform to within 1.25\% (Pokora (2009)). A conical convergent nozzle was used, based on the geometry described in the study of Power et al. (2004), and is shown in figures 1(b) and $1(\mathrm{c})$. The nozzle exit diameter $\left(D_{j}\right)$ was $40 \mathrm{~mm}$ with internal and external face convergence angles of $6^{0}$ and $11^{0}$ and an exit plane lip thickness of $1.2 \mathrm{~mm}$. To ensure the nozzle was located in the centre of the water tunnel a supporting cross-shaped structure was used (figure 1(b)) and allowed the jet axis to be aligned to within $1^{0}$ with respect to the tunnel walls.

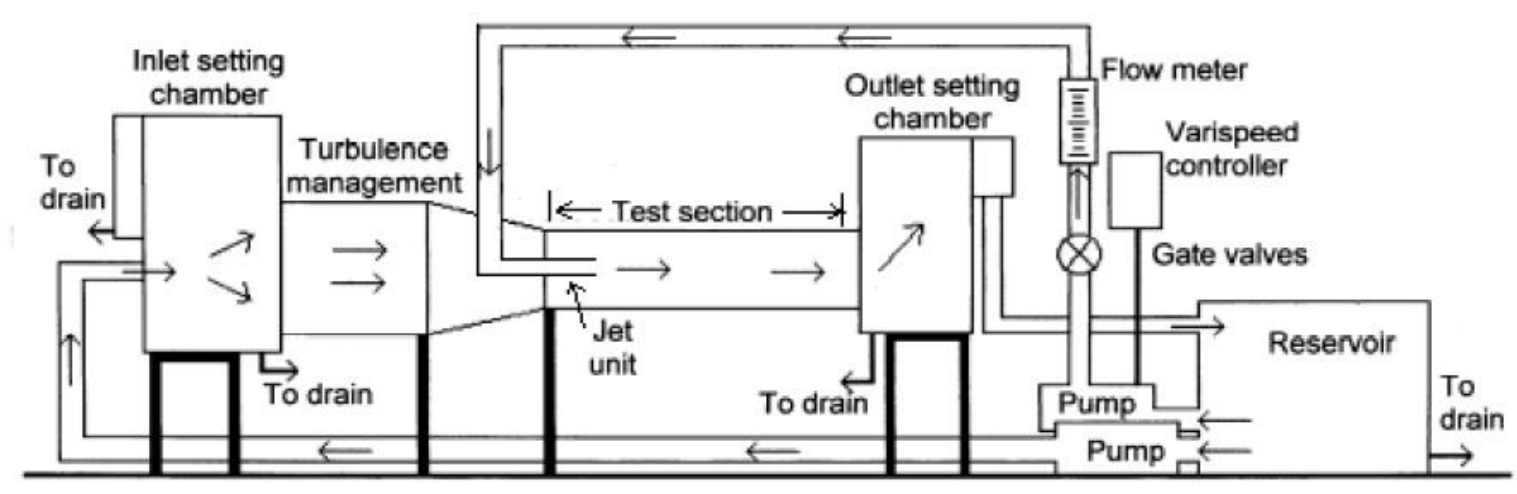

(a)

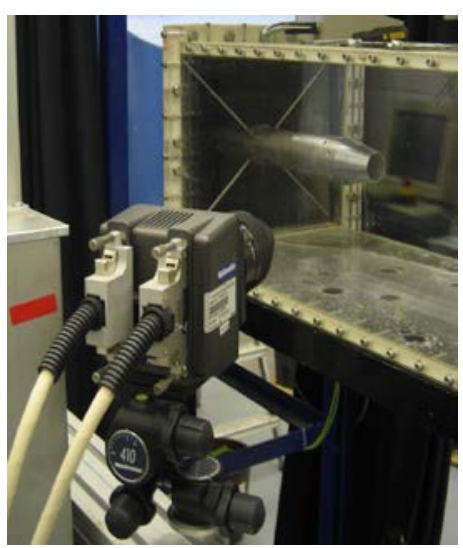

(b)

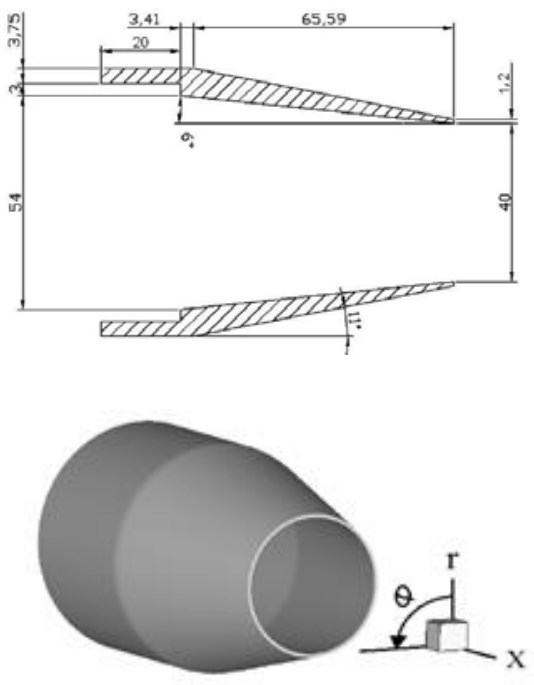

(c)

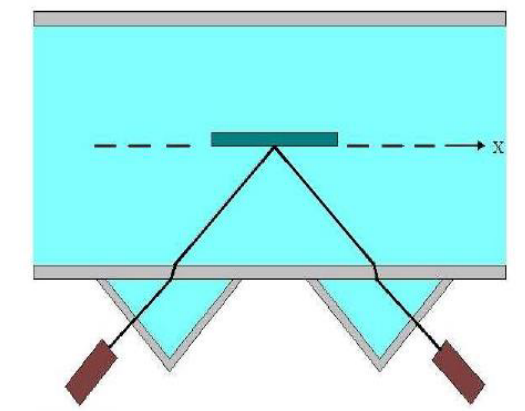

CAMERA 1

FIGURE 1 (Colour online) (a) Water tunnel; (b)PIV camera and jet nozzle inside water tunnel; (c) nozzle geometry and co-ordinate definition; (d) x-r plane 3C-PIV water jacket arrangement 
The bulk average nozzle exit velocity was $1 \mathrm{~m} / \mathrm{s}$, giving a Reynolds number $\operatorname{Re}_{D}=4 \times 10^{4}$. The airflow data in the literature reviewed in the Introduction have generally been taken at higher Reynolds numbers than this (typically from $\operatorname{Re}_{\mathrm{D}} \sim 1 \times 10^{5}$ to $\sim 5 \times 10^{5}$ ) and at Mach numbers varying from $0.3-0.9$. However, if attention is focussed on flow and in particular on turbulence data (as opposed to acoustic measurements), when plotted nondimensionally no significant Reynolds number or Mach number effects were observed in any of these published studies. In addition, the experiments of Ricou and Spalding (1961) concluded that the entrainment process for turbulent free jets became independent of $\mathrm{Re}_{\mathrm{D}}$ above $2 \times 10^{4}$. The recent measurements of Fellouah et al. (2009) in the jet near-field $(\mathrm{x} / \mathrm{D}<30)$ supported this view, noting that for $\mathrm{Re}_{\mathrm{D}}>2 \times 10^{4}$ velocity spectra in the shear layer displayed a clear inertial sub-range indicating a one-way coupling of large turbulent scales and viscosity influenced small scale motions. Their measurements showed no significant dependency on $\operatorname{Re}_{\mathrm{D}}$ for the centreline mean velocity decay rate for $\operatorname{Re}_{\mathrm{D}}$ above $10^{4}$ in the near-field region studied. The peak turbulent stresses in the shear layer showed less than $5 \%$ change for $\operatorname{Re}_{\mathrm{D}}$ above $10^{4}$ and even then only in the immediate vicinity of the nozzle. The conclusion from these investigations is that Reynolds number effects are predominantly associated with the transition of the near wall flow from a wall boundary layer (which does contain a region of direct viscous-influenced flow) to a turbulent free shear layer. This process begins at nozzle exit and takes place typically within the first 1 or 2 nozzle diameters of axial length; after that the shear layer and jet development are dominated by turbulence processes that are not directly set by molecular viscosity. Thus, the Reynolds number for the present experiment was chosen to be sufficiently high that the measurements will be relevant to the high Re nozzle flows found in practice.

Figure 1 (c) indicates the cylindrical polar $(x, r, \theta)$ co-ordinate system, with origin at the nozzle exit centreline, used to present the data. For 3C-PIV measurements, waterfilled prisms were attached to the tunnel walls to ensure the optical axis of the PIV cameras was orthogonal to the first air/Perspex/water interface (figure 1d). Placing the jet in a confined environment will affect the development of the flow to a greater or lesser extent dependent upon geometry and test conditions. A RANS-based CFD study (k- $\varepsilon$ turbulence model) was undertaken (Pokora (2009)) in order to minimise these effects. The mixing of the jet and external co-flow is determined by the entrainment rate, which is controlled primarily by the ratio of jet to co-flow velocities. If the flow development is to be similar to that of a free jet, this velocity ratio must be as large as possible. However, if the water tunnel co-flow does not supply sufficient entrainment flow for the whole downstream development of the jet, an adverse pressure gradient is created in the tunnel that can cause flow recirculation in the external co-flow. By adjusting the ratios of nozzle diameter/tunnel size and jet/tunnel velocity this confinement effect can be minimised, by ensuring a value of the Craya-Curtet number (Craya and Curtet (1955)) such that $C t>1.0$. Thus if $U_{j}, U_{c f}$ and $U_{m}$ are the jet, co-flow, and fully mixed bulk average velocities, and $D_{j}$ , $D_{c f}$ are the diameters of jet and co-flow areas (equivalent circular area for the co-flow), $C t$ is given by:

$$
C t=\frac{U_{m}}{\left[\left(U_{j}^{2}-U_{c f}^{2}\right)\left(\frac{D_{j}}{D_{c f}}\right)^{2}+\frac{\left(U_{c f}^{2}-U_{m}^{2}\right)}{2}\right]}
$$


A co-flow velocity of $0.18 \mathrm{~m} / \mathrm{s}$ was found to avoid any recirculation or jet-flapping induced unsteadiness at the far downstream end of the test section. Comparison of confined water flow measurements with free jet air flow data are provided in the Results section below to indicate that the data measured here are representative of turbulent subsonic free jet behaviour. Further, by providing all required boundary conditions appropriate to the present experimental set-up, comparable CFD simulations may be carried out for direct comparison with the current measurements.

\subsection{Instrumentation}

The PIV system used consisted of a pair of LaVision High Speed Star 4 (HSS4) 1024x1024 pixel resolution cameras (at 10bit dynamic range) capable of frame rates up to $1 \mathrm{kHz}$ double frames at full resolution, at which the on-board camera memory enables a sample size of 3 secs, allowing a total of 3072 instantaneous vector fields to be recorded. Lenses of focal lengths from $24 \mathrm{~mm}$ to $105 \mathrm{~mm}$ were used depending on the desired FoV. The light source was New Wave double-cavity high speed Nd/YLF Pegasus laser with wavelength $527 \mathrm{~nm}$ and power of $10 \mathrm{~mJ} / \mathrm{pulse}$ at a repetition rate of $1 \mathrm{kHz}$ with pulse duration $6 \mathrm{~ns}$. The PIV cameras and lasers were controlled by LaVision's DaVis (v7.2) software and Programmable Timing Unit (PTU). The laser beams are fired through an optical arm followed by a divergent lens to form a laser sheet with adjustable thickness $0.5 \mathrm{~mm}$ $2.5 \mathrm{~mm}$. Laser head and both cameras were mounted on a Dantec Dynamics 3-axis traverse enabling system alignment to $\sim 0.2 \mathrm{~mm}$. PIV calibration used the LaVision twolevel calibration plate placed within the tunnel. For 2C-PIV only a single camera is used, viewing orthogonal to the water tunnel side wall; for stereo 3C-PIV measurements, where two cameras viewed the flow field at an oblique angle through the tunnel side wall, water jackets with $45^{0}$ sides were fitted to the sides of the tunnel (figure $1 \mathrm{~d}$ ) to ensure the lenses of both cameras were located parallel to the first air/Perspex boundary in order to reduce any effects incurred due to refractive index changes. Scheimpflug lenses were included to tilt the image plane with respect to the orientation of the camera lens and light sheet. This arrangement widens the focused range although has the side-effect of introducing a strong perspective distortion; the factor of magnification is also no longer constant across the complete field of view. Both of these are accounted for during image calibration. A focal length lens of $105 \mathrm{~mm}$ was used for 2C-PIV measurements, producing a FoV of $25 \mathrm{mmx} 25 \mathrm{~mm}$; the rationale for this choice of small FoV size is discussed below. For 3CPIV in an X-r plane both cameras viewed through the tunnel side wall; if the laser sheet was oriented in the $r-\theta$ plane, one camera was placed on each side of the illuminated plane. For the $r-\theta$ orientation, the $105 \mathrm{~mm}$ lenses were equipped with $2 \mathrm{x}$ teleconverters, and this together with a viewing arrangement - i.e. with cameras and Scheimpflug lenses on either side of the light sheet plane - leads to some elongation due to perspective effects and in the $r-\theta$ plane the FoV width was increased from 25 to $30 \mathrm{~mm}$. Figure 2 displays the different orientations of the instrumentation for 2C- or 3C-PIV and in different planes. 


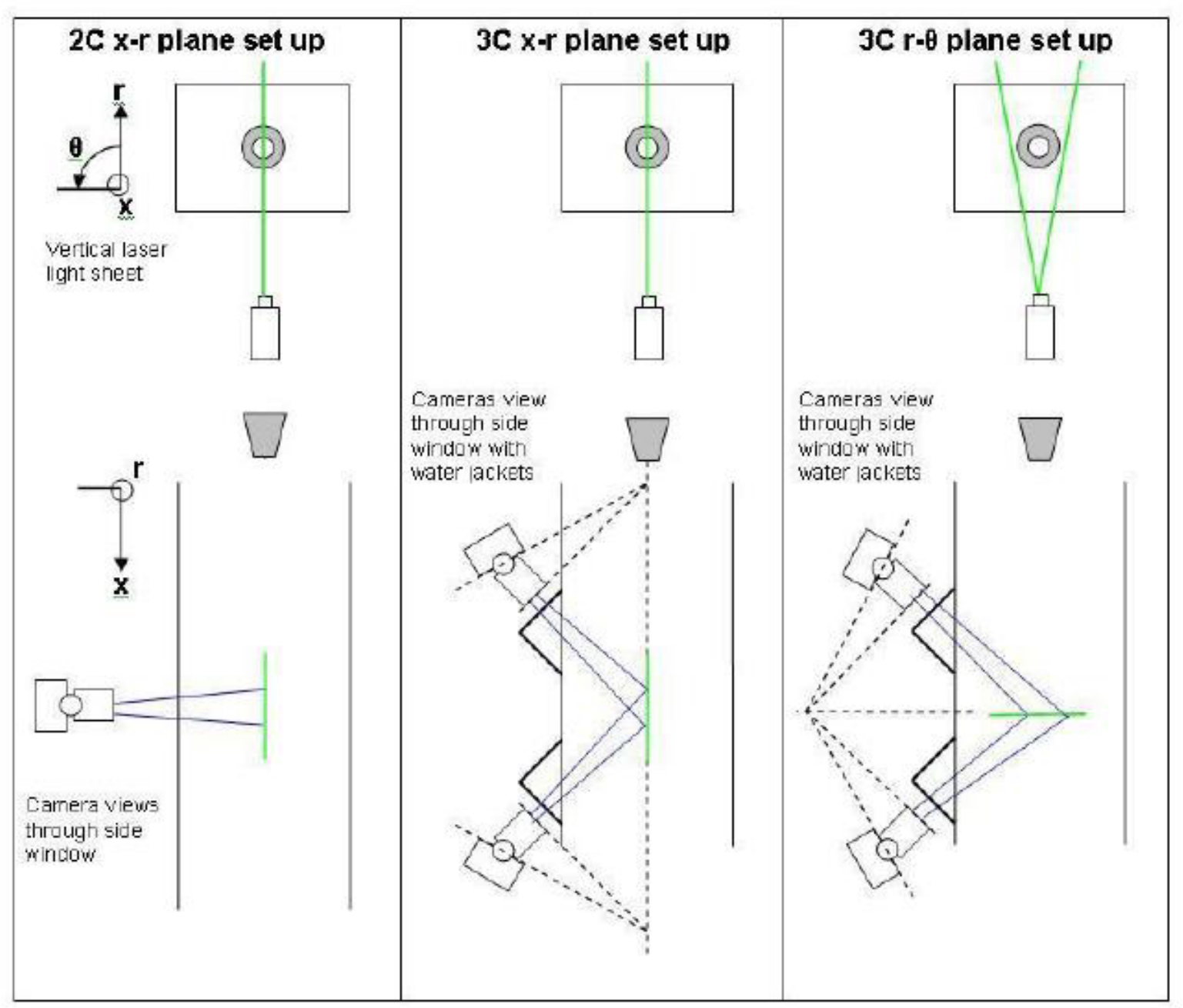

FIGURE 2 (Colour online) Camera and laser locations for various measurement configurations

\subsection{Data Definitions}

The usual Reynolds decomposition is adopted to separate time-mean (statistically stationary) and fluctuating components of the instantaneous velocity field:

$$
U_{i}^{i n s t}\left(\underline{x}_{P}, t\right)=U_{i}\left(\underline{x}_{P}\right)+u_{i}^{\prime}\left(\underline{x}_{P}, t\right) \quad \text { where: } U_{i}\left(\underline{x}_{P}\right)=\frac{1}{N_{\text {samp }}} \sum_{n=1}^{N_{\text {samp }}} U_{i}^{i n s t}\left(\underline{x}_{P}, t_{n}\right)
$$

The time mean of any variable is calculated at the centre of each PIV interrogation window $\left(\underline{x}_{P}\right)$ by averaging over the total number of PIV frames sampled, as shown above for the mean velocity. Single point Reynolds stresses are defined as usual, e.g. for the rms of the Reynolds normal stresses (an overbar now indicating averaging over frames):

$$
u_{i}^{r m s}\left(\underline{x}_{P}\right)=\sqrt{\overline{u_{i}^{\prime 2}}}\left(\underline{x}_{P}\right)
$$

For spatio-temporal characteristics of the turbulence, the definitions adopted for the normalised $2^{\text {nd }}$ - and $4^{\text {th }}$-order two-point, two-time correlations have been given above. Simpler correlations of interest may be obtained by setting specific values of $\underline{\eta}$ and $\tau$, e.g. 
the auto-correlation function is obtained by setting $\underline{\eta}=0$ and the spatial correlation function by setting $\tau=0$. Integral length scales may be extracted from the spatial correlations, e.g. at $2^{\text {nd }}$-order level:

$$
{ }^{k} L_{i j}\left(\underline{x}_{P}\right)=\int_{0}^{\infty} R_{i j}\left(\underline{x}_{P}, \eta_{k}, 0\right) d \eta_{k}
$$

where $\mathrm{k}$ indicates the component of the separation vector along which integration is carried out; the streamwise integral scale is evaluated as ${ }^{1} L_{11}$ and the transverse integral scale as ${ }^{2} L_{11}$. Note that in practice these integral scales are evaluated up to the first zero crossing of the correlation function (as in Fleury et al. (2008)), and for accuracy it was always ensured that the evaluation point $\underline{x}_{P}$ was sufficiently far from the edge of the FoV that the correlation decreased to zero within the FoV in the direction of integration.

Similarly, the $2^{\text {nd }}$-order correlation function may be used to analyse the fluctuating energy distribution. The energy spectrum (Power Spectral Density PSD) may be obtained from a Fourier transform of the auto-correlation function:

$$
E_{i j}\left(\underline{x}_{P}, \omega\right)=2 \int_{0}^{\infty} R_{i j}\left(\underline{x}_{P}, 0, \tau\right) \cos (\omega \tau) d \tau
$$

Noise due to low sampling rates can produce noisy spectra; to avoid this, all spectra presented below were calculated as the mean of spectra at the 9 interrogation cells surrounding a given location, given the fine spatial resolution used this was considered acceptable.

\section{Image Processing}

\subsection{Data Quality Checks}

The standard checks to monitor the quality of the conversion of raw PIV images to vector fields were carried out. An Adaptive Multi-Pass technique was used with final cell size $32 \times 32$ pixels; to increase vector density $50 \%$ overlap of PIV cells was adopted.

\section{Vector validation}

The quality of the vector map produced was quantified and improved using the standard $\mathrm{Q}$-factor (a 'pass' test of $\mathrm{Q}>2$ was adopted to indicate a valid vector) and 'nearneighbour' tests (see Westerweel et al. (1996)). All vectors removed were replaced to maintain a continuous field. For 2C-PIV data the number of $1^{\text {st }}$ choice vectors was greater than $98 \%$; for 3 C-PIV this was $80 \%$ but the number of $1^{\text {st }}$ or $2^{\text {nd }}$ choice vectors was $95 \%$.

\section{$\underline{\text { Sub-cell Filtering Estimation and Correction }}$}

The method of Spencer and Hollis (2005) has been used to estimate the level of turbulence energy lost due to sub-cell spatial filtering, to correct for this, and also to choose the most appropriate size for the PIV FoVs at various locations in the jet (shear layer growth changes the integral scale and hence permits larger PIV interrogation window/cell sizes and hence FoVs at downstream locations). The size of the PIV cell effectively performs a low-pass filter on the measured displacement of the seeding particles; fluctuating energy of any scales smaller than the cell size is filtered out. HoestMadsen and Nielsen (1995) produced an estimate of this loss of turbulence in PIV measurements expressed via a predicted curve for the ratio of measured turbulence rms to true rms as a function of the ratio of PIV cell size $(\Delta x)$ to the local integral scale of 
turbulence (L). For example if $\Delta x / L=1$, a loss in measured turbulence of around $30 \%$ was predicted to occur. Using this curve to correct PIV measurements is problematic since apriori the integral length scale (and its variation across the FoV) is unknown. In addition, the Hoest-Madsen and Nielsen prediction assumed homogenous isotropic turbulence, which is rarely the case in practice. Spencer and Hollis (2005) suggested an improved approach for turbulence correction based on: (i) a correction curve for turbulence error versus $\Delta \mathrm{x} / \mathrm{L}$ generated from measurements in shear flows (boundary layer, free shear, separated, and wake flows) to avoid assumptions about the nature of the turbulence, (ii) use of an LDA technique to obtain the true rms since this does not suffer from the same spatial filtering problem and was compared with PIV measurements on a series of ever decreasing size FoVs, and (iii) calculation of the integral scale from the PIV data using eqn. (2.4), again on a series of ever-decreasing FoV size, with the most accurate value taken from the smallest FoV to create a second correction curve for the integral length scale. Figure 3 presents the two correction curves taken from Spencer and Hollis (2005).

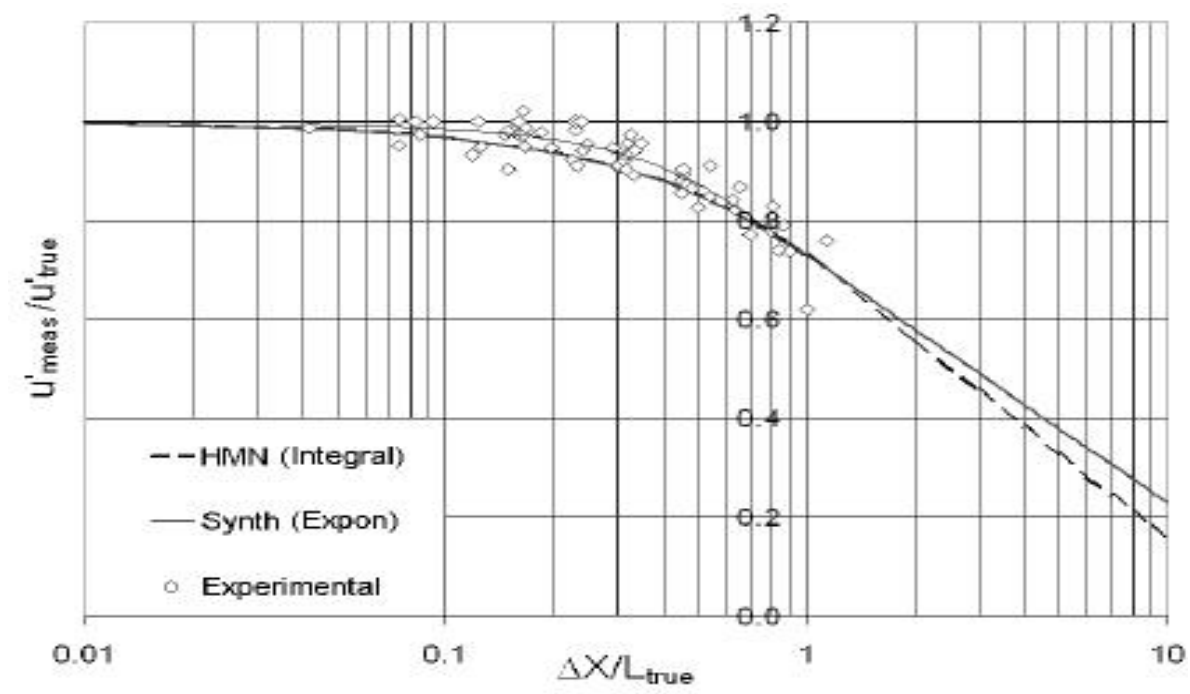

(a)

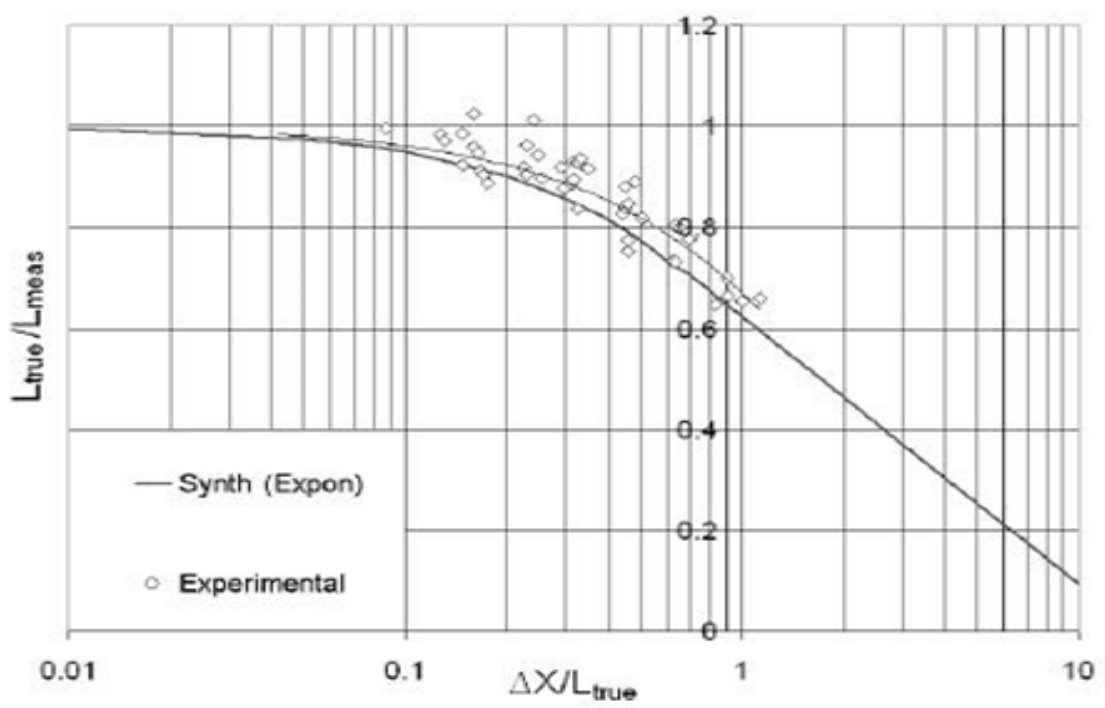

(b)

FIGURE 3 Sub-cell filtering correction curves for (a) rms and (b) integral scale (from Spencer and Hollis (2005)) 
These two curve-fits have been used in the present work to estimate corrected PIV measured quantities $u_{\text {meas }}^{\text {rms }}$ and $L_{\text {meas }}$. Note that iteration between these curves is needed, given $u_{\text {meas }}^{\text {rms }}$ and $L_{\text {meas }}$, to complete the correction (for details, see Spencer and Hollis (2005)).

As an illustration of the use of this method, figure 4 shows the ratio between measured radial rms values taken in the present study and corrected values using the above methodology for a PIV with FoV centred on selected points in the jet shear layer at 4 axial locations. Two FoV sizes were explored: $100 \mathrm{mmx} 100 \mathrm{~mm}$ and $60 \mathrm{mmx} 60 \mathrm{~mm}$; the axial rms indicated lower errors (see Pokora (2009)), so results for radial rms are shown. The contour maps show that for the larger FoV the error is significantly larger - up to 25\% loss of energy is indicated at the first measurement location (thinnest shear layer). This

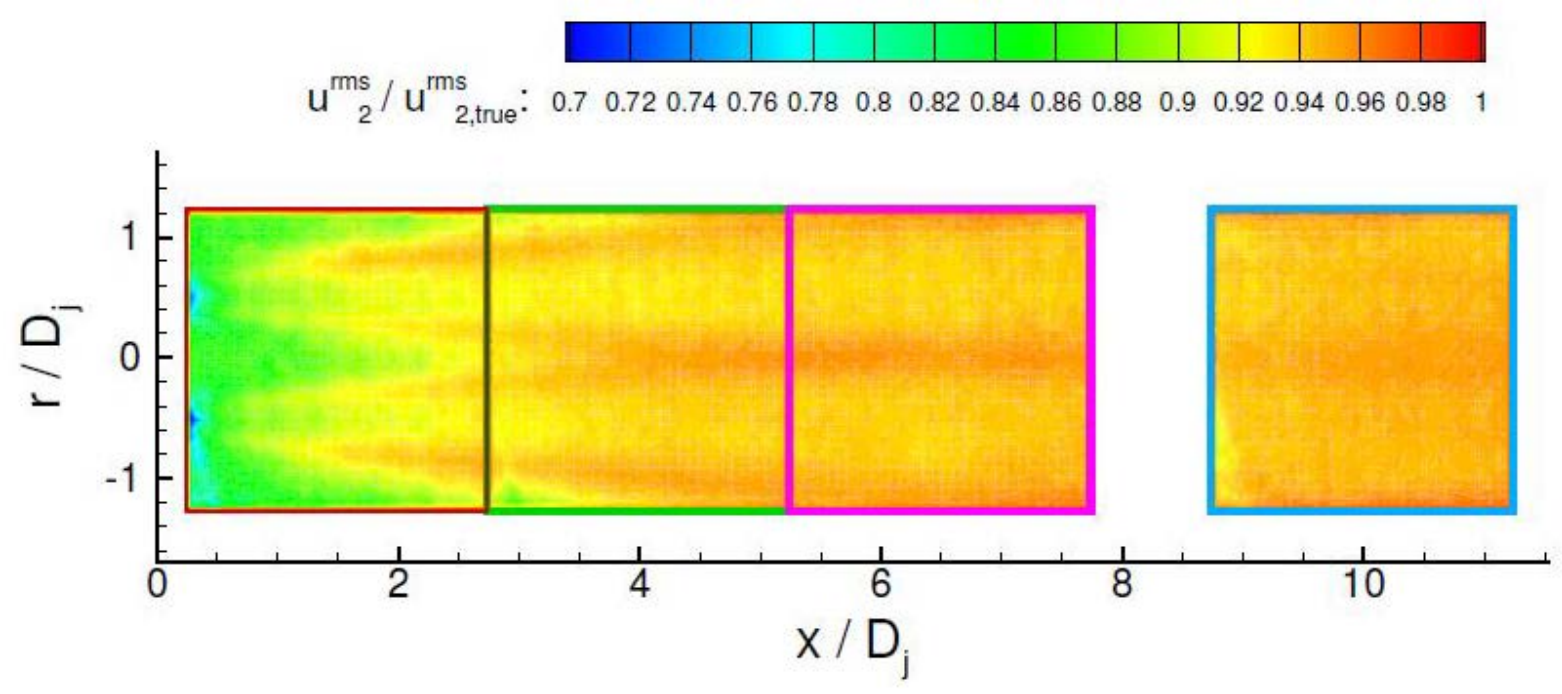

(a)

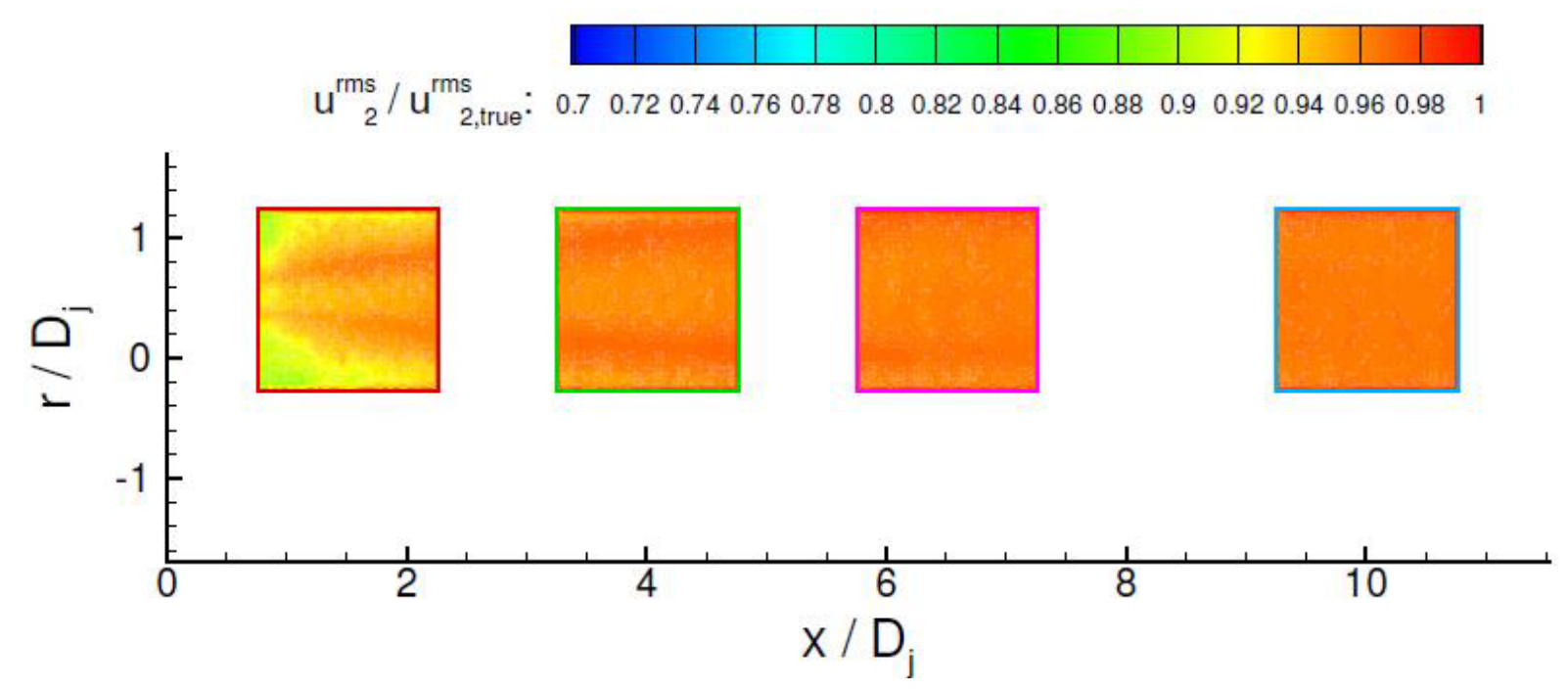

FIGURE 4 (Colour online) Radial rms error maps for FoV sizes: (a) 100x100mm, (b) 60x60mm

error is reduced for the smaller FoV to less than 10\%. This shows the importance of adjusting the FoV size to match the local integral scale. At $x / D_{j}=1.5$, tests showed that to reduce the error to less than $5 \%$, a FoV of $25 \mathrm{mmx} 25 \mathrm{~mm}$ was necessary. Table 1 shows the 
errors evaluated using the Spencer and Hollis methodology for a FoV of $25 \mathrm{mmx} 25 \mathrm{~mm}$ for both axial and radial rms, and this is the reason this FoV size was chosen for the 2C-PIV data to ensure optimum spatial and temporal resolution.

$\begin{array}{ccccc}\text { RMS } & \mathbf{x} / \mathbf{D}_{\mathbf{j}}=\mathbf{1 . 5} & \mathbf{x} / \mathbf{D}_{\mathbf{j}}=\mathbf{4 . 0} & \mathbf{x} / \mathbf{D}_{\mathrm{j}}=\mathbf{6 . 5} & \mathbf{x} / \mathbf{D}_{\mathbf{j}}=\mathbf{1 0 . 0} \\ \mathrm{u}^{r m s} & 3.9 \% & 1.9 \% & 1.6 \% & 1.2 \% \\ \mathrm{v}^{r m s} & 3.2 \% & 2.7 \% & 2.8 \% & 2.1 \%\end{array}$

TABLE 1 Error levels, axial and radial rms, FoV of $25 \mathrm{mmx} 25 \mathrm{~mm}$

Finally, Table 2 compares the spatial resolution achieved in the current experiments compared to the previous studies of Fleury et al. (2008) and Wernet (2007). On the basis of this, the current measurements contain significantly lower levels of spatial filtering of turbulence than in previous work.

\begin{tabular}{cccccc}
\hline Authors & $\mathbf{D}_{\mathbf{j}}(\mathbf{m m})$ & FoV(pixels) & FoV( $\left.\mathbf{D}_{\mathbf{j}}\right)$ & $\begin{array}{c}\text { Cell size } \\
(\mathbf{p i x e l s})\end{array}$ & $\begin{array}{c}\text { Cell size } \\
\mathbf{( m m})\end{array}$ \\
Wernet (2007) & 50.8 & $1024 \times 144$ & $2.95 \times 0.37$ & $32 \times 32$ & $4.69 \times 4.22$ \\
Fleury et al.(2008) & 38 & $1280 \times 1024$ & $2.2 \times 1.8$ & $32 \times 32$ & $2.09 \times 2.14$ \\
Pokora and McGuirk & 40 & $1024 \times 1024$ & $0.62 \times 0.63$ & $32 \times 32$ & $0.78 \times 0.78$
\end{tabular}

TABLE 2 Spatial resolution achieved in various PIV studies

\section{Choice of FoVs}

As well as considering FoV size for high accuracy, the need to measure 2-point correlations at large enough separation vectors to capture the whole extent of the correlation function must be considered. Measurements were again conducted using various FoV sizes centred on points where the correlations/length scales measured could be compared with data from literature. Sufficiently large FoVs were also required to ensure the correlations would decay to zero within the FoV in both in-plane directions (the integral length scales measured by Fleury et al. (2008) were used to guide this). The FoVs used are shown in figure 5 for 2 locations in the shear layer on the nozzle lipline upstream of potential core end $\left(r / D_{j}=0.5\right.$, at $\left.x / D_{j}=1.5,4.0\right), 2$ locations near potential

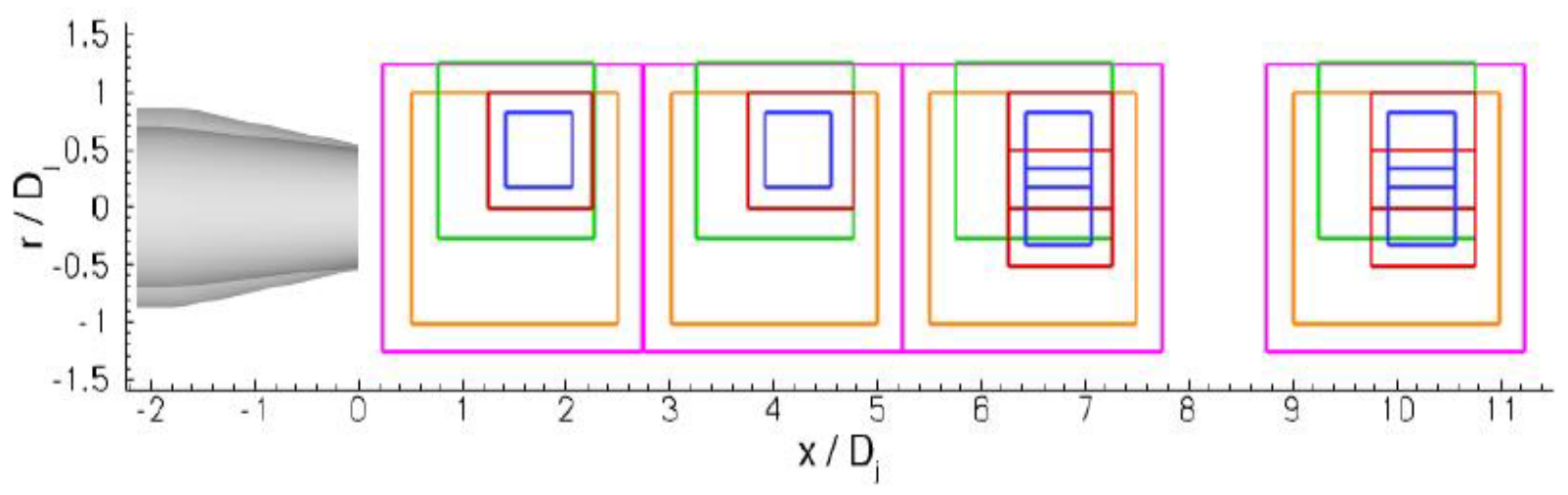

FIGURE 5 (Colour online) Location of different size FoVs within jet region 
core end $\left(x / D_{j}=6.5\right.$, at $\left.r / D_{j}=0.5,0.0\right)$, and 2 further downstream $\left(x / D_{j}=10\right.$, at $r / D_{j}=0.5$, 0.0 ). At each location 5 FoVs were used varying in size from $25 \mathrm{mmx} 25 \mathrm{~mm}$ to $100 \mathrm{~mm} \times 100 \mathrm{~mm}$, with data taken both at $50 \mathrm{~Hz}$ (for longer sampling time) and $1 \mathrm{kHz}$ (for maximum temporal resolution) - in each case with 3072 samples gathered.

Figure 6 shows measurements of the axial rms velocity fluctuation along the jet centreline $\left(r / D_{j}=0.0\right)$ and the nozzle lipline $\left(r / D_{j}=0.5\right)$ for all FoV sizes. On the centreline within the potential core region, there can be no shear-generated turbulence; the measured turbulence should be no larger than at nozzle exit (i.e. low $\sim 0.01 \mathrm{~m} / \mathrm{s}$ ) and decay downstream until the inner edge of the lipline shear layer meets the centreline. Figure 6a however shows considerable and increasing apparent turbulence in the region $0.0<x / D_{j}<5$ (a feature also observed by Power at al. (2004)). A change in gradient is seen

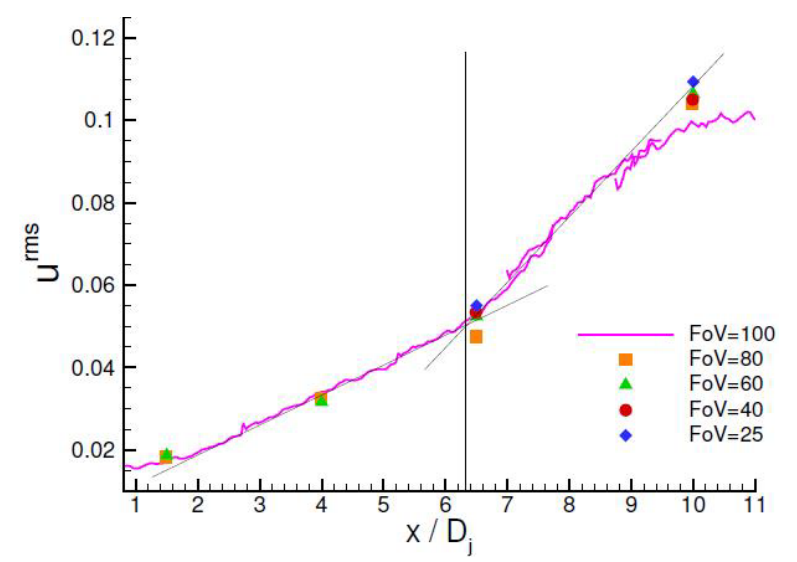

(a)

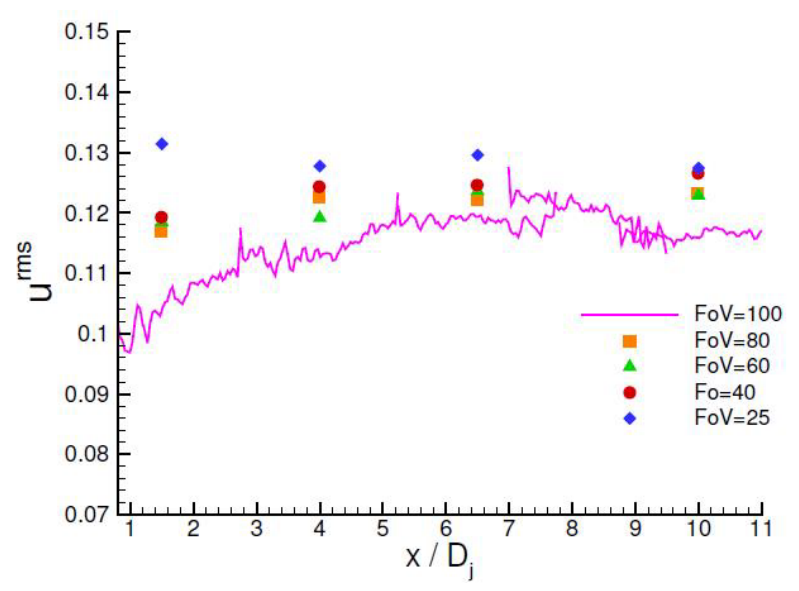

(b)

FIGURE 6 (Colour online) Axial development of $\mathrm{u}_{\mathrm{rms}}$ for $(\mathrm{a})$ jet centreline and (b) jet lipline

at $x / D_{j}=6.3$; this indicates the arrival of 'true' shear-generated turbulence on the centreline and identifies the potential core length LPC. In the Mach $0.75, \mathrm{Re}=8 \times 10^{5}$ airflow measurements of Power at al. (2004) $\mathrm{LPC}_{\mathrm{PC}}$ was measured as $6.5 \mathrm{D}_{\mathrm{j}}$, providing further evidence of the marginal influence of both Mach number and Reynolds number (if this is high enough) on turbulence development in the present flow. On the centreline upstream of the potential core end the PIV system is actually measuring irrotational unsteadiness induced by the streamwise convection of large turbulent structures in the shear layer, which cause the edge of the inviscid potential core region (and hence the velocity within it) to fluctuate. Only after potential core end does the FoV size influence results, with smaller FoVs producing slightly higher rms. A better indication of large FoV size causing turbulence error is seen in the lipline measurements (figure 6b). At small $\mathrm{x} / \mathrm{D}_{\mathrm{j}}$ the $\mathrm{FoV}$ size has a large effect (thin shear layer relative to FoV size); further downstream as the integral length scale grows, less sub-cell filtering is evident. The data on the lipline become sensibly independent of FoV beyond $x / D_{j}=4$, where the difference between FoVs of $40 \mathrm{~mm}$ and $25 \mathrm{~mm}$ is less than $3 \%$. On the other hand the scatter is seen to increase at larger $x / D_{j}$, since the integral timescale also grows, implying fewer statistically independent samples in fixed sample duration (3 seconds). 


\section{Statistical accuracy}

Statistical accuracy is dependent on the number of independent samples; this was the reason data were also collected at $50 \mathrm{~Hz}$. Convergence is slower for locations further downstream (larger turbulence timescales) and for $2^{\text {nd }}$ moment quantities. Figure 7 illustrates a typical example from the current measurements using different sub-sample sizes of the full data set for a point on the centreline at the furthest downstream station and for the radial rms. Standard error curves for $95 \%$ and $99 \%$ confidence limits are also included, with the convergence rate from sub-sampling agreeing well with these.

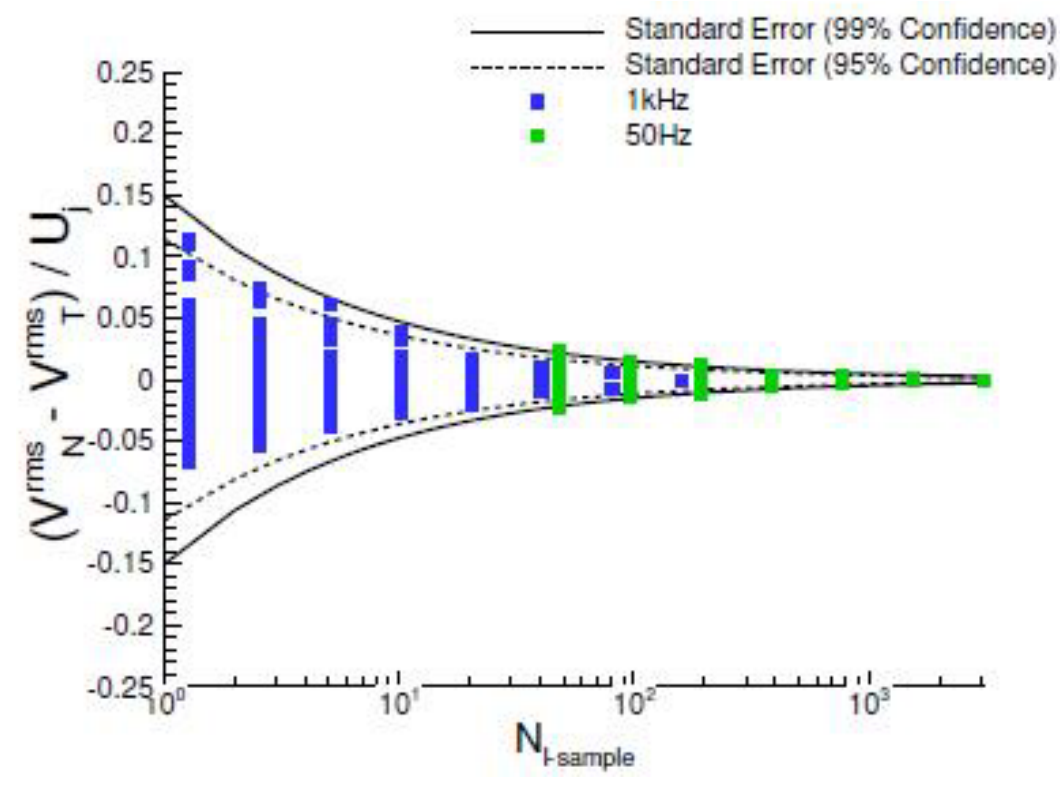

FIGURE 7 (Colour online) Statistical sampling check: $v_{r m s}$ at $\mathrm{x} / \mathrm{D}_{\mathrm{j}}=10.0, \mathrm{r} / \mathrm{D}_{\mathrm{j}}=0.0$

\section{Results}

\subsection{C-PIV Data - Comparison with subsonic jet airflow data}

Single point measurements were first made using 2C-PIV to provide evidence to support the use of PIV data gathered in a water flow experiment as representative of subsonic isothermal airflow jet turbulence. Measured PIV mean flow statistics in the x-r plane are shown in figure 8 via radial profiles at $x / D_{j}=4$ of the axial mean and rms velocity compared to the LDA data of Lau et al. (1979) $\left(\operatorname{Re}=3 \times 10^{5}-10^{6}\right.$, Mach $\left.=0.28,0.9\right)$, Morris and Zaman (2010) $\left(\mathrm{Re}=3 \times 10^{5}\right.$, Mach $\left.=0.26\right)$, and the HWA data of Harper-Bourne (2003) $\left(\operatorname{Re}=2 \times 10^{5}\right.$, Mach $\left.=0.18\right)$. The good agreement in the mean profile with airflow data at higher Re indicates the behaviour of the jet shear layer is essentially unaffected by 


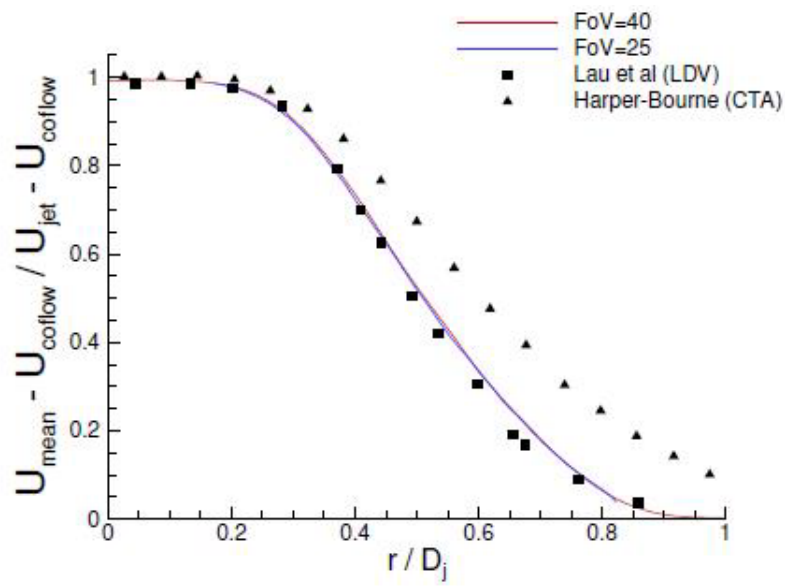

(a)

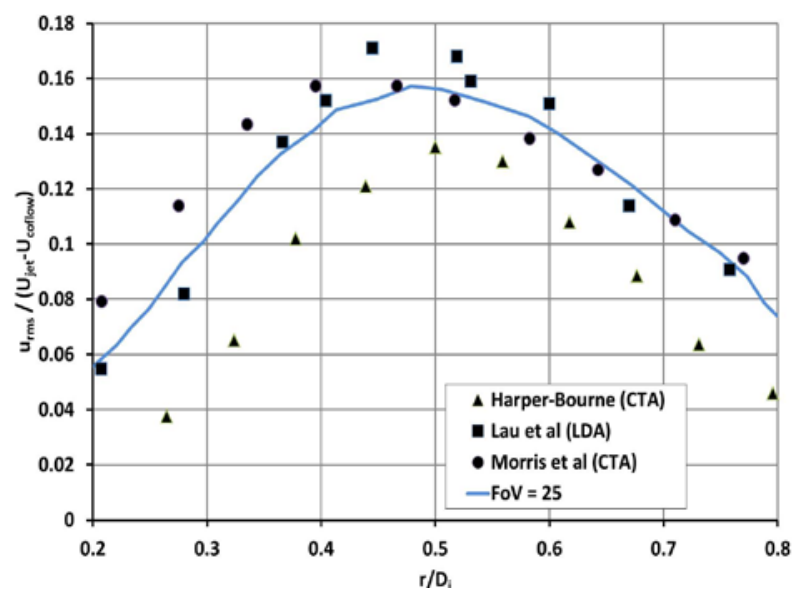

(b)

FIGURE 8 (Colour online) Radial profiles of (a) mean and (b) rms axial velocity at $x / D_{j}=4.0$

the use of water compared to airflow, the tunnel confinement, or the value of Re. The PIVmeasured axial turbulence rms profile also lies in between the profiles measured by Lau et al. (1979), Morris and Zaman (2010), and Harper-Bourne (2003). The consensus data set established by the review of many free jet data by Bridges and Wernet (2010) at various Reynolds numbers and subsonic Mach numbers indicated a peak axial rms value of 0.16 at this axial location; the current data are in good agreement with this value.

As noted in the Introduction, numerous airflow experiments over a range of subsonic jet Mach numbers have provided turbulence statistical information based on 2point measurements. The most important physical parameter on this category is the turbulence length scale information at locations within jet shear layers provided by various authors (Liepmann and Laufer (1947), Davies et al. (1962), Harper-Bourne (2003), Fleury et al. (2008)). Turbulence length scales are evaluated by integrating the measured $2^{\text {nd }}$-order spatial correlation functions for both axial and radial fluctuations and with respect to both axial and radial separation vectors (as defined in Eqn. (2.4) above). Figure 9 shows data obtained from the current 2C-PIV measurements (symbols) compared to the 2C-PIV-measured data presented in Fleury et al. (2008); information on the $2^{\text {nd }}$-order correlation functions $R_{i j}$ measured in the present 2C-PIV study will be presented below in Section 3.4. It can be seen that the values obtained from the present water flow experiments are in excellent agreement for all four length scales with the airflow data obtained using PIV and for a two subsonic Mach numbers (0,6 and 0.9) by Fleury at al. (2008). This provides strong support to the argument that the 2-point correlations and associated turbulent structures present in low speed water flow experiments, when suitably normalised, are identical to subsonic jet airflow characteristics. 


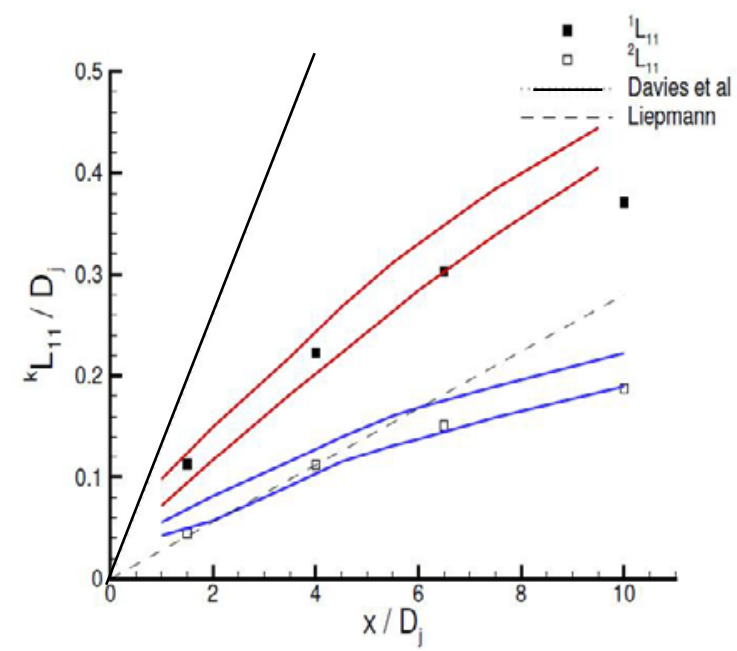

(a)

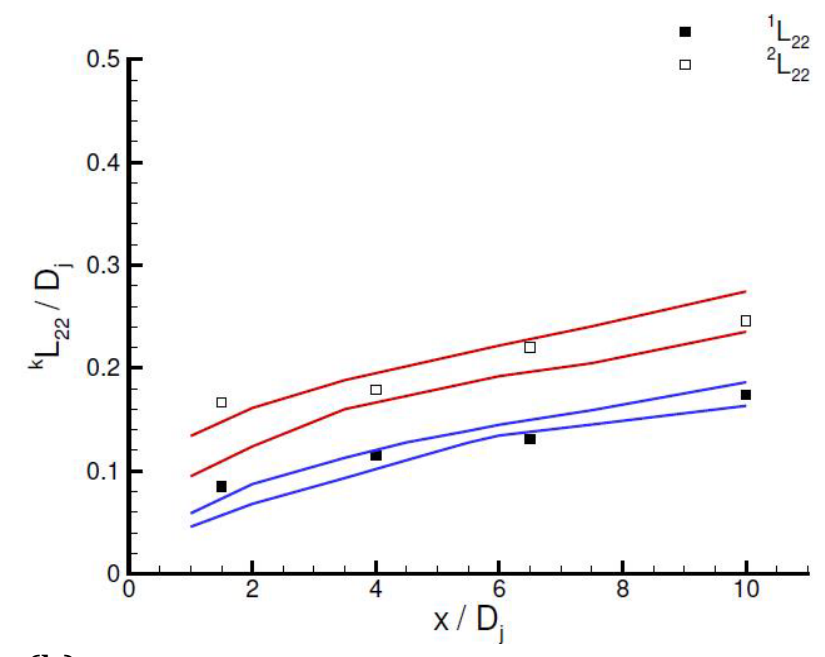

(b)

FIGURE 9 (Colour online) Axial (a) and radial (b) lengthscales at $\mathrm{r} / \mathrm{D}_{\mathrm{j}}=0.5$

Red and blue lines indicate range of data in experiments of Fleury et al. (2008)

This examination of 1-point and 2-point turbulence statistics shown was considered sufficient to demonstrate that the combination of a water flow experiment and PIV measurements at $1 \mathrm{kHz}$ (with a suitably chosen small FoV) can achieve spatial and temporal resolution comparable to the high temporal resolution HWA methods as used in subsonic airflow experiments, and can thus deliver data on the important turbulence correlations which characterise the aerodynamic noise source.

\subsection{C-PIV Data - Preliminary Measurements}

The PIV system parameters (FoV size, vector validation, number of frames etc.) were optimised as described above using 2C-PIV for the X-r plane velocity field. When moving to 3C-PIV, measurements were first carried out to judge the quality of the $3 \mathrm{C}$ data compared to the standard established after 2C-PIV optimisation, since some deterioration and increase in measurement error was to be expected. Figure 10 shows a comparison of 3C-PIV and 2C-PIV single point data for mean axial velocity (top) and axial rms (bottom) at various axial locations. In general the $2 \mathrm{C}$ - and $3 \mathrm{C}$-PIV data for the mean velocity are in very good agreement. For measurements of the turbulent fluctuations it would be expected that $3 \mathrm{C}$ data taken with the laser sheet in the $\mathrm{x}-\mathrm{r}$ plane, when the streamwise velocity is an in-plane component, would be more accurate than $3 \mathrm{C}$ measurements with the laser sheet in the $r-\theta$ configuration (see figure 2 above), when the streamwise velocity is an out-of-plane component. In addition to this, as noted in section 2.2 above, the $r-\theta$ plane FoV is actually $20 \%$ larger than the x-r plane FoV, which would be expected to lead to increased loss of turbulence. It is therefore surprising that in figure 10, whilst the measurements of rms with both 2C- and 3C-PIV systems are fairly close, the $r-\theta$ data are in better agreement with the 2C measurements than the x-r data, which show a $20 \%$ increased peak value compared to $2 \mathrm{C}$-data. This arouses suspicion that increased noise in the $3 \mathrm{C}$ data was compensating in the $r-\theta$ measurements for extra turbulence loss and thus showing false 'good' agreement with the $2 \mathrm{C}$ data. 

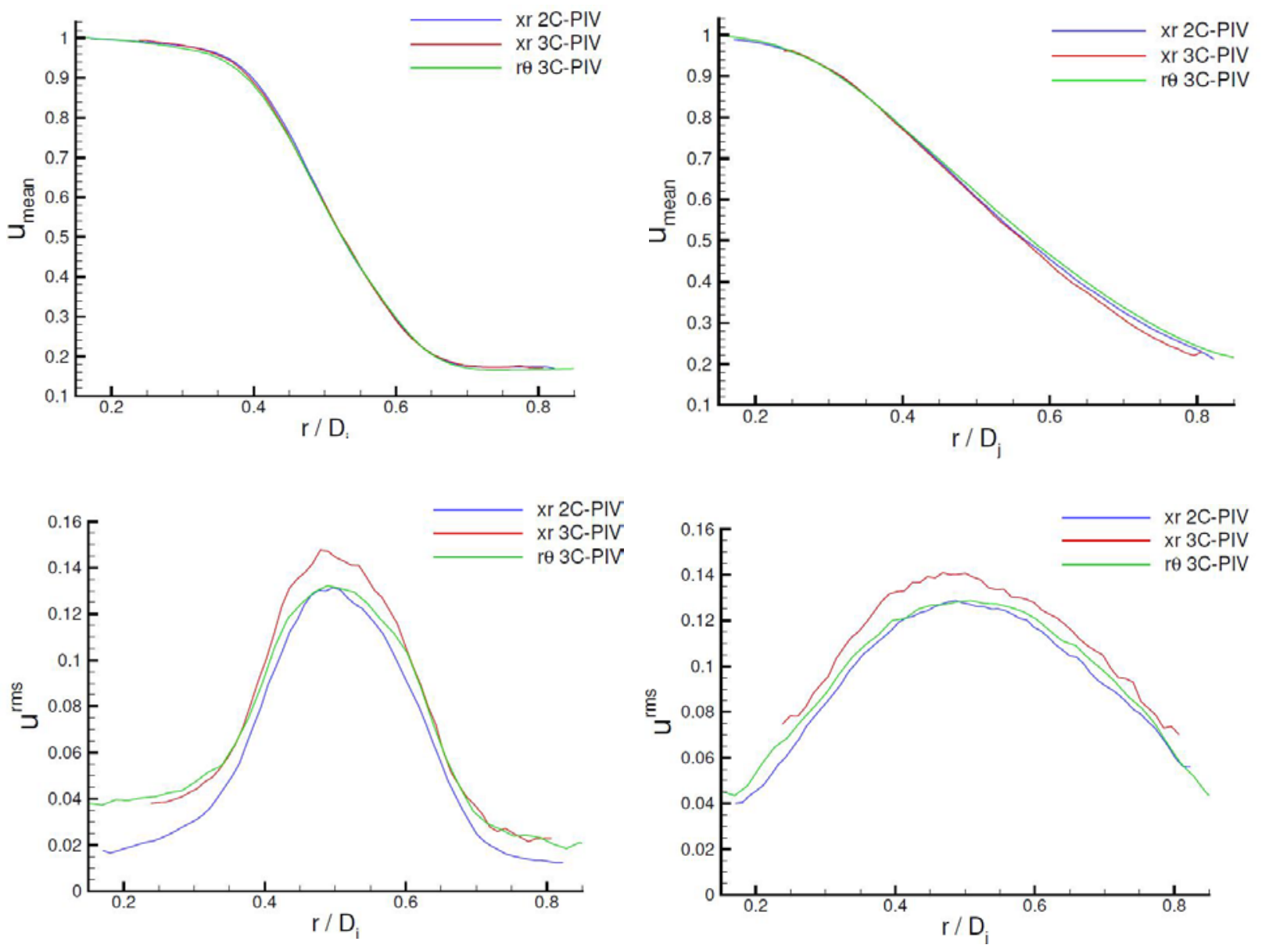

FIGURE 10 (Colour online) Radial profiles: axial mean (top) and rms (bottom) at x/D = 1.5 (left), 4.0 (right) - different PIV configurations

Examination of data for the instantaneous velocity vector field produced by 3C-PIV $\mathrm{x}$-r plane measurements indicated a larger number of spurious vectors that 'survived' the vector post-processing filter than in 2C-PIV data taken in the same plane and with the same PIV set-up (an example of this is presented below in figure 14). This reinforced the suspicion that the $3 \mathrm{C}$ data contained a noise source not in the $2 \mathrm{C}$ data, and that this was occurring at high frequencies. Although stronger filtering helped to reduce the number of spurious vectors, some remained, and it was felt stronger filtering was too crude an approach, since this may adversely affect the energy content in frequency regions which were not affected by increased instrumentation noise. An alternative method for removal of possible high frequency noise was therefore required. The method adopted was suggested by close examination of the $2 \mathrm{C}$ and $3 \mathrm{C}$ measured frequency spectra. Figure 11 presents 2C-PIV and 3C-PIV Power Spectral Density (PSD) measurements for the axial 

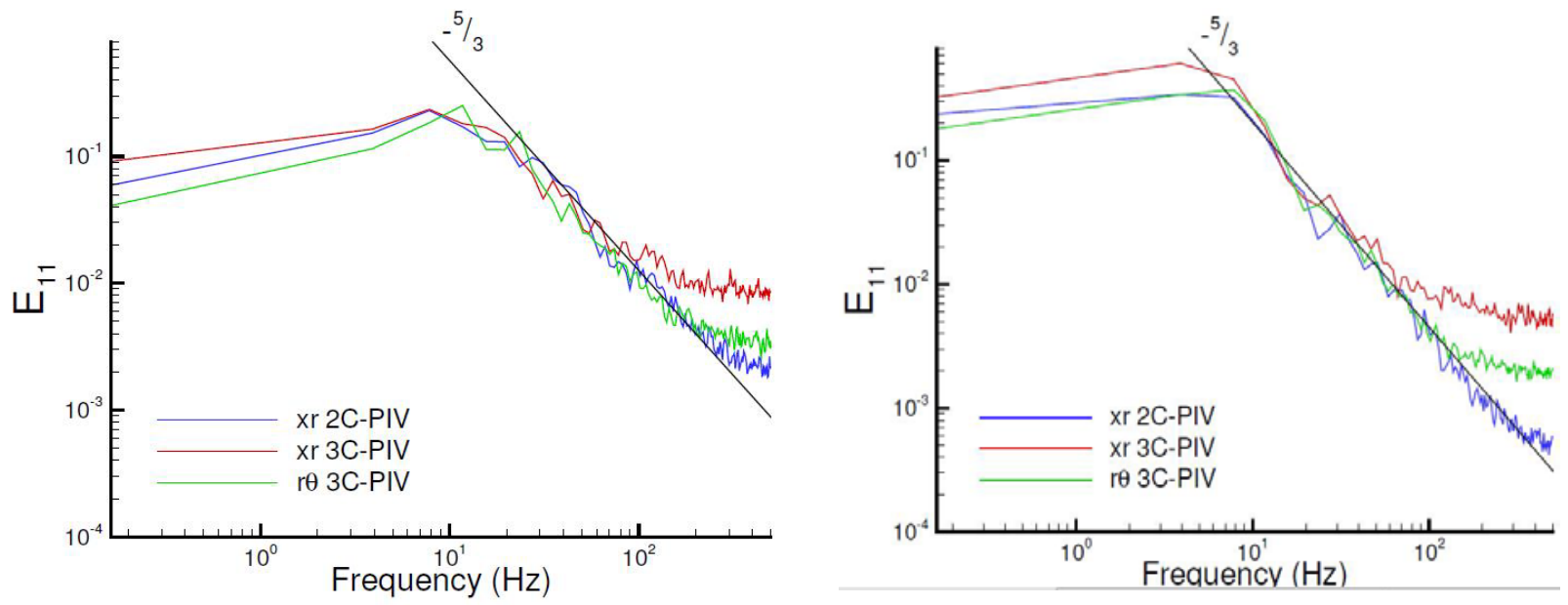

FIGURE 11 (Colour online) PSD of fluctuating axial velocity: $r / D_{j}=0.5$ and $x / D_{j}=1.5$ (left) and 10.0 (right)

velocity fluctuation at a point in the centre of the shear layer and at two axial locations; the smallest $25 \mathrm{~mm}$ FoV data area shown. It is clear that, whilst the $2 \mathrm{C}$ data follow a $-5 / 3$ equilibrium energy cascade well for 2 decades of energy decrease, at frequencies greater than $100 \mathrm{~Hz}$ there is evidence of increased noise contamination in the 3C-PIV data causing high frequency 'lift', with the measured data lying above the equilibrium line. This is particularly strong in the 3C-PIV data for the x-r plane and becomes stronger with downstream distance where any FoV size-based measurement errors become weaker rather than stronger. A POD-based filter method was therefore developed to correct for this high frequency contamination and this is outlined in the following section.

\subsection{C-PIV Filtering/Correction Method}

It is clearly important that any correction method should not affect the raw data in spectral regions not believed to be contaminated by noise. Any filtering should only remove spurious contributions to the fluctuating energy. Proper Orthogonal Decomposition (POD) was an obvious possible approach, since this orders spatial modes in terms of decreasing contribution to the overall energy captured. Time-resolved POD (Weitao et al. (2003)) uses the time-series information at each 3C-PIV interrogation cell to produce an optimal energy-based decomposition into modes. By adopting a low energy modal cut-off filter, it is possible to filter high frequency noise contributions. POD is first used to analyse the velocity field and identify a hierarchy of spatial modes; from this information a series of velocity fields may be reconstructed containing, for example, only the first $\mathrm{N}$ modes (larger $\mathrm{N}$ thus corresponds to weaker filtering). An example of such a reconstruction using different numbers of POD modes, from just the first 2 to 6, 42, 313 1nd finally all 3072 modes; the fraction of the total kinetic energy these contain is also shown in figure 12 . 


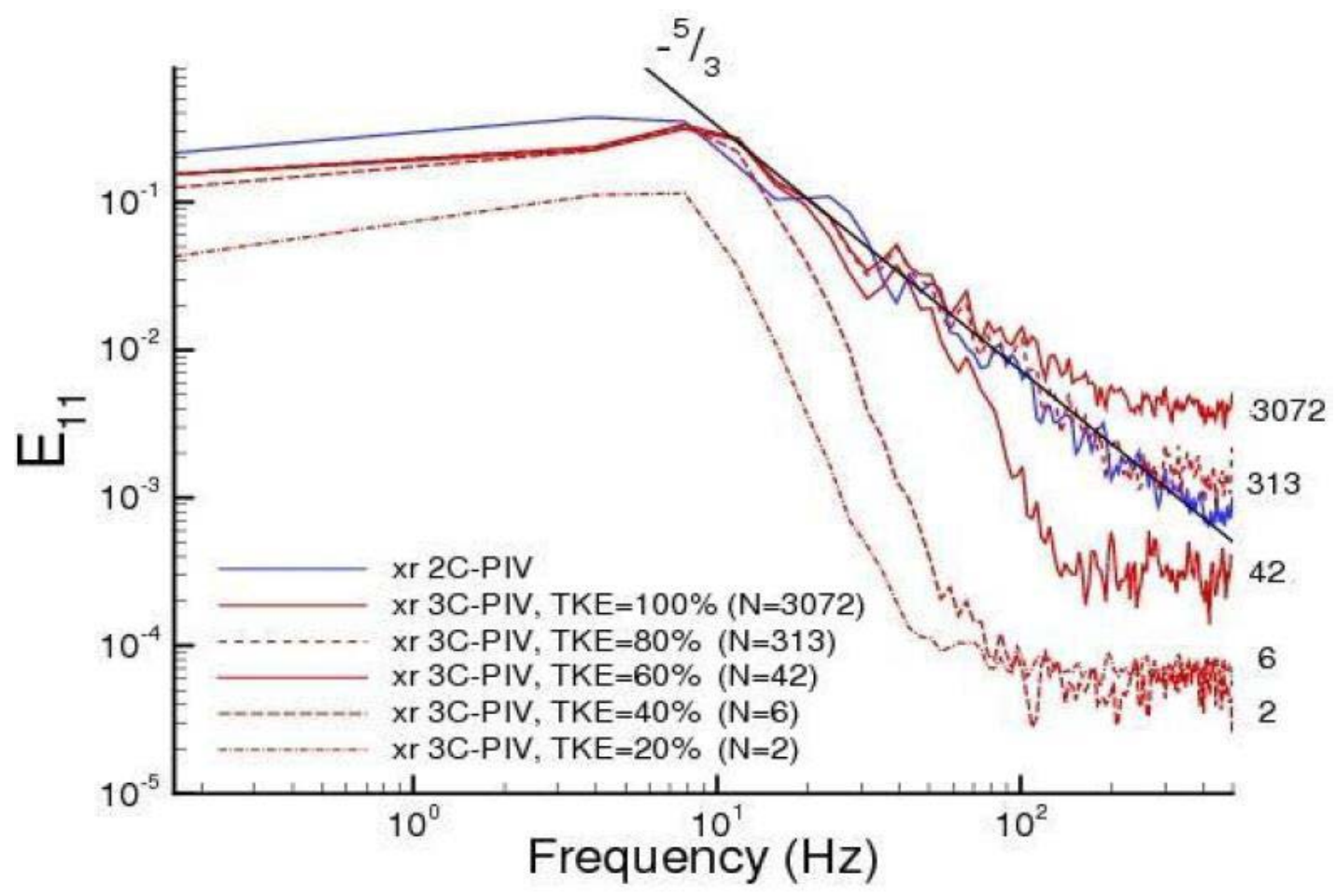

FIGURE 12 (Colour online) Effect of POD mode number on reconstructed PSD (blue - 2C_PIV)

Figure 12 illustrates clearly that if a large amount of energy is filtered (small $\mathrm{N}$ ) then the original PSD is altered not only in the high frequency region where spurious high frequency lift is suspected (the 2C-PIV spectrum was used to identify this) but also at frequencies believed to be accurate (good agreement between 3C-PIV and 2C-PIV data). However, if a larger $\mathrm{N}$ is selected (say 313), filtering only $20 \%$ of the original energy, then the resulting PSD is very close to the 2C-PIV PSD over practically the whole range of temporally resolved frequencies. The original 3C-PIV spectrum $(\mathrm{N}=3072)$ departs from the 2C-PIV data at $\sim 50 \mathrm{~Hz}$.; if the $20 \%$ POD filtered spectrum is used, this is close to the 2C-PIV spectrum up to $\sim 300 \mathrm{~Hz}$, a six fold improvement.

The method adopted was to select a number $\mathrm{N}^{*}$ POD modes, where $\mathrm{N}^{*}$ was defined to maximise the cumulative energy fraction and provide the best fit to the 2C-PIV spectrum. The reconstructed energy content may be viewed as original energy minus a fraction assumed to be high frequency (spurious) noise. $\mathrm{N}^{*}$ was varied for each point within the FoV and for each location in the jet. An illustration of the method is provided in figure 13. The amount of spurious energy to be removed $(\Delta \mathrm{E})$ is defined by two limits of integration in frequency space (figure 13 (right)). The upper limit was set to the highest resolved frequency $(500 \mathrm{~Hz})$; the lower limit was set by the value of $\mathrm{N}^{*}$ chosen as described above. Note that departure from a $-5 / 3$ spectrum could have been used and would have produced similar results. 

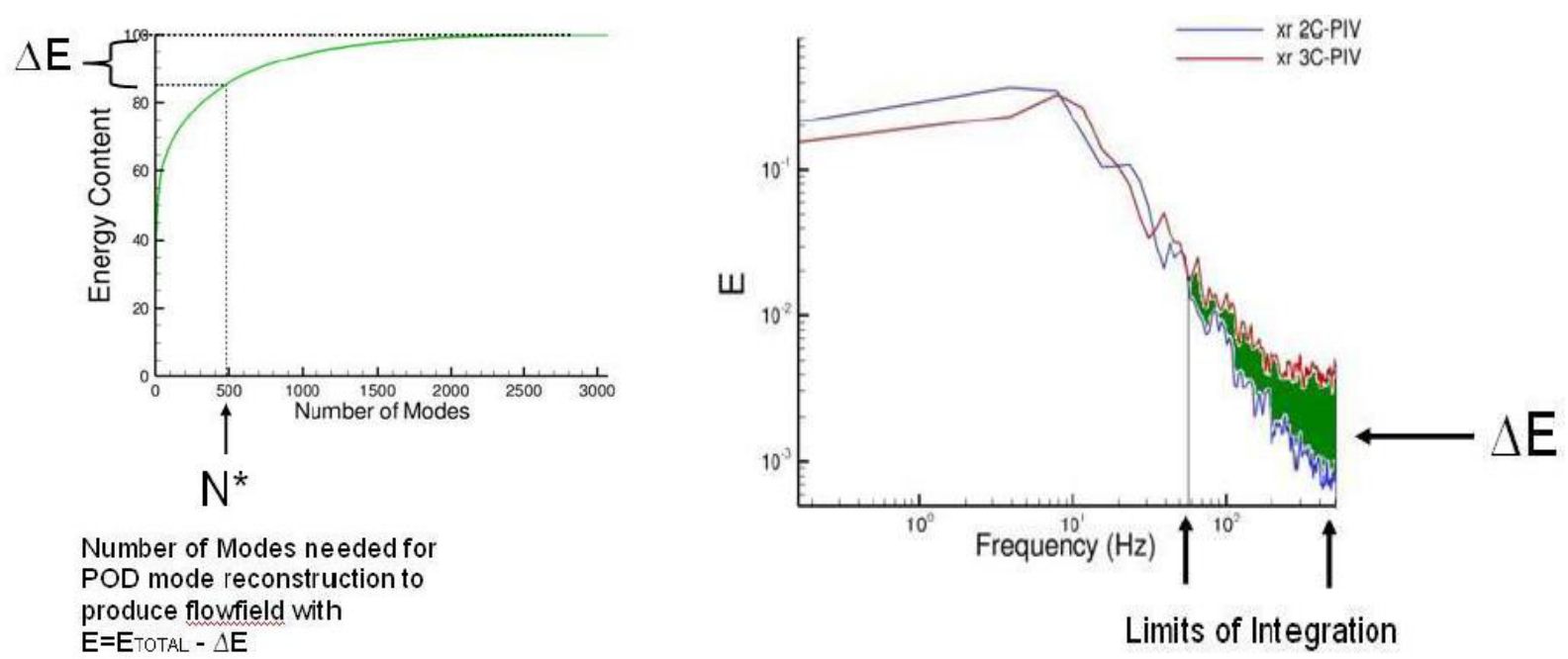

FIGURE 13 (Colour online) Illustration of POD correction method

Using this method, Table 3 shows for various points the amount of spurious energy identified which was typically removed from the x-r plane 3C-PIV measurement to generate corrected 3C-PIV spectra; $\mathrm{r}-\theta$ planes treated were similarly.

\begin{tabular}{|c|c|c|c|c|c|}
\hline Location & $\Delta \mathrm{E}(\%)$ & Location & $\Delta \mathrm{E}(\%)$ & Location & $\Delta \mathrm{E}(\%)$ \\
\hline $\mathrm{x} / \mathrm{D}_{\mathrm{j}}=1.5 \mathrm{r} / \mathrm{D}_{\mathrm{j}}=0.5$ & 29.3 & $\mathrm{x} / \mathrm{D}_{\mathrm{j}}=6.5 \mathrm{r} / \mathrm{D}_{\mathrm{j}}=0.0$ & 10.7 & $\begin{array}{c}x / D_{j}=10.0, r / D_{j}= \\
0.0\end{array}$ & $12.1^{\circ}$ \\
\hline $\mathrm{x} / \mathrm{D}_{\mathrm{j}}=4.0 \mathrm{r} / \mathrm{D}_{\mathrm{j}}=0.5$ & 20.5 & $\mathrm{x} / \mathrm{D}_{\mathrm{j}}=6.5 \mathrm{r} / \mathrm{D}_{\mathrm{j}}=0.5$ & 14.0 & $\mathrm{x} / \mathrm{D}_{\mathrm{j}}=\underset{0.5}{10.0, \mathrm{r} / \mathrm{D}_{\mathrm{j}}=}$ & 22.3 \\
\hline
\end{tabular}

TABLE 3 Spurious energy content identified and removed from 3C-PIV measurements

To illustrate the consequence of this procedure on instantaneous frames of PIV vectors, figure 14 shows, for the plane $x / D_{j}=6.5$ : (i) the original vector field captured by the $3 \mathrm{C}$ PIV and (ii) the reconstructed field using the described POD filtering.
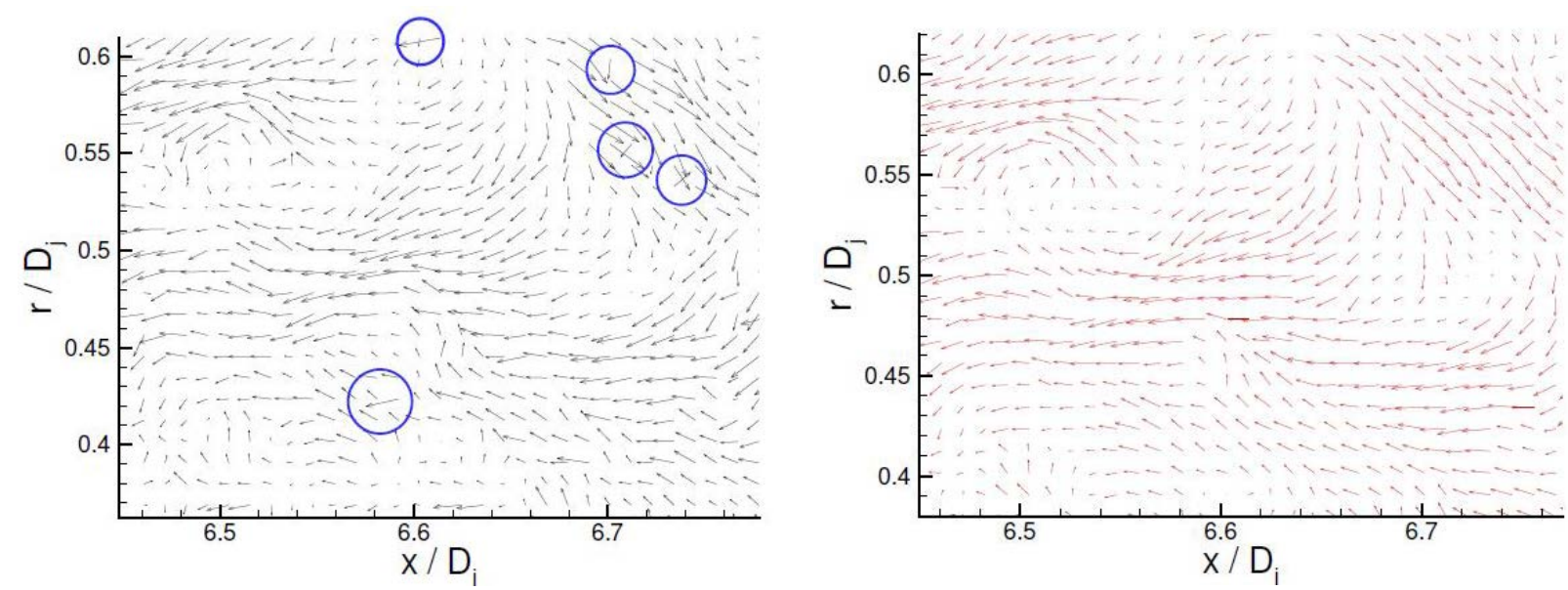

FIGURE 14 (Colour online) Example of instantaneous vector fields at $\mathrm{x} / \mathrm{D}_{\mathrm{j}}=6.5$,

(i) original, (ii) original + POD correction 
Figure 14(i) contains the spurious vectors not removed during normal PIV vector postprocessing, which are the main source of the noise in the 'raw' 3C-PIV spectrum. Modification of the post-processing parameters to remove these was explored but caused undesirable removal of energy in PSD regions which were in good agreement with the 2CPIV data. Figure 14(ii) shows the result of the POD correction method described - all spurious vectors have been removed and the larger coherent structures have become more defined. Figure 15 presents the effect of filtering on the PSD of the $\mathrm{N}^{*}$ mode reconstructed field. The reduction in the high frequency noise is exactly as desired, and comparison between the 2C-PIV spectra and the filtered spectra (labelled 3C-PIV*) is excellent for all locations within the important shear layer region. All 3C-PIV x-r and $r-\theta$ plane data have been filtered using this method to produce the results reported below.
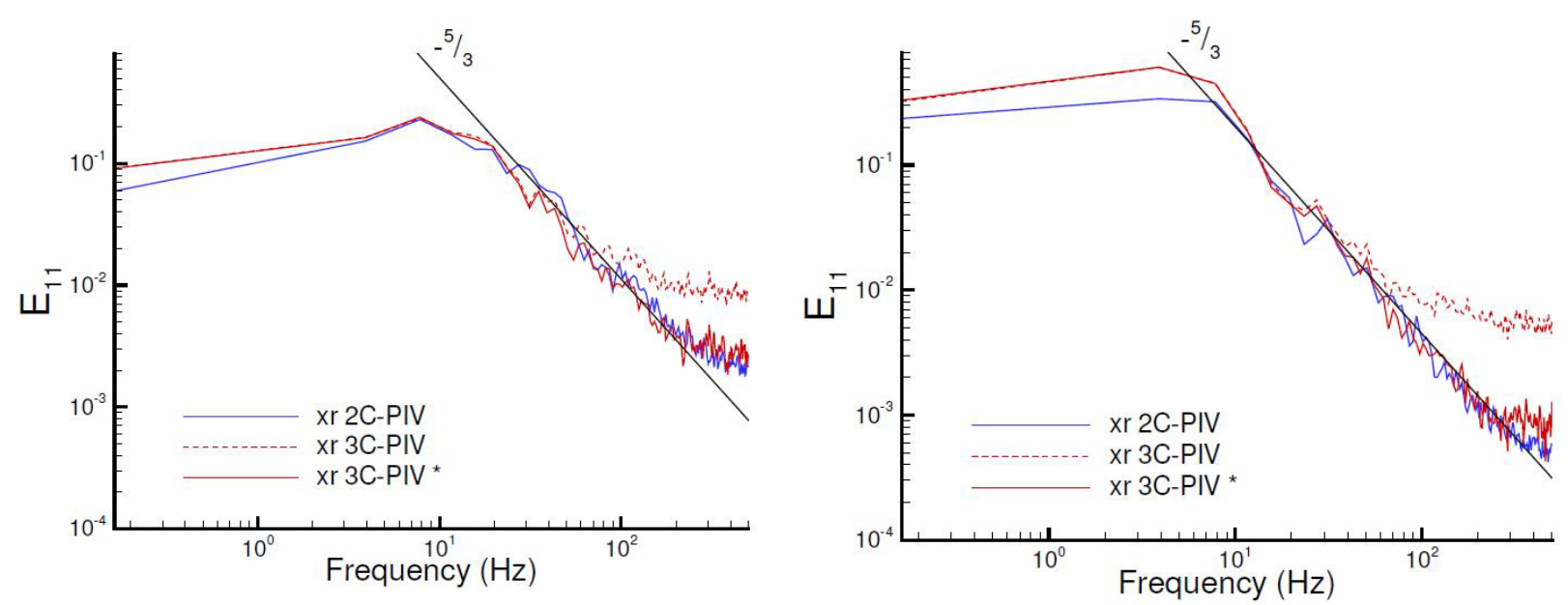

FIGURE 15 (Colour online) Power spectra of axal velocityc- comparison of 2C-PIV, 3C-PIV and 3C*-PIV (left) $x / D_{j}=1.5, r / D_{j}=0.5$, (right) $x / D_{j}=10.0, r / D_{j}=0.5$

Radial profiles of axial rms as discussed above in figure 11 were examined again using the 3C-PIV* dataset at all measured shear layer locations. Figure 16 shows the comparison between 2C-PIV, original 3C-PIV, and filtered 3C-PIV*data. The improvement in the agreement between $2 \mathrm{C}$ and $3 \mathrm{C}^{*}$ data provides strong evidence in support of the PODbased correction methodology. At $\mathrm{x} / \mathrm{D}_{\mathrm{j}}=1.5$ the $3 \mathrm{C}^{*}$ profile lies almost exactly on the $2 \mathrm{C}$ profile; further downstream improved agreement is noticeable. Increased statistical noise is caused by the removal of some data, but the filtered results are still a significant improvement on the single point $2^{\text {nd }}$ order turbulence statistics. 


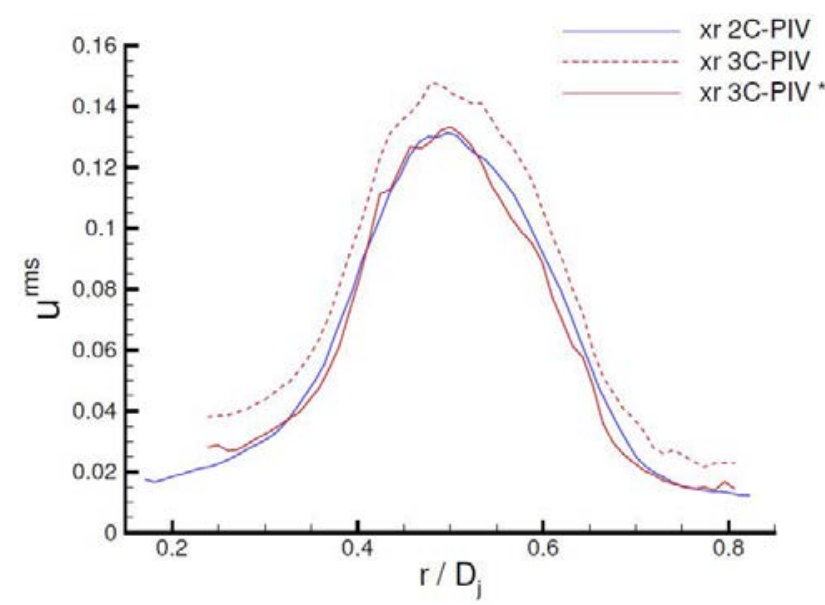

(a)

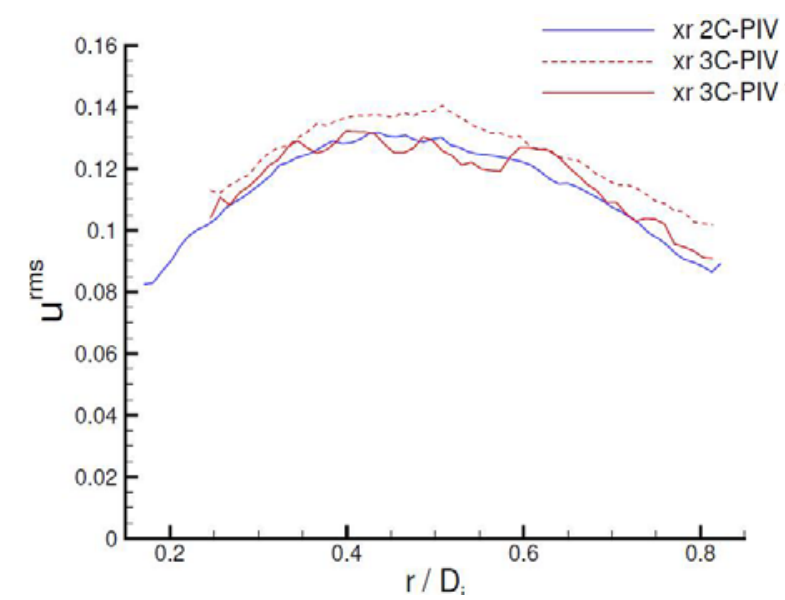

(c)

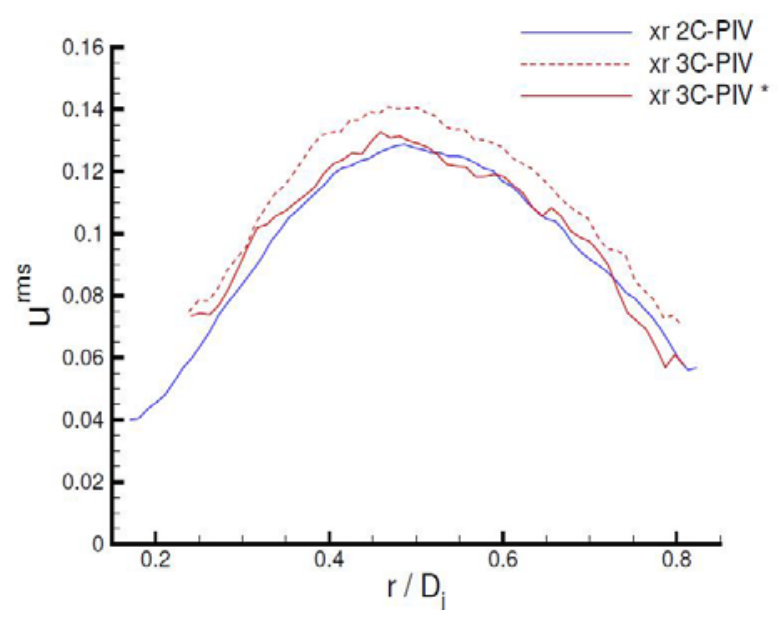

(b)

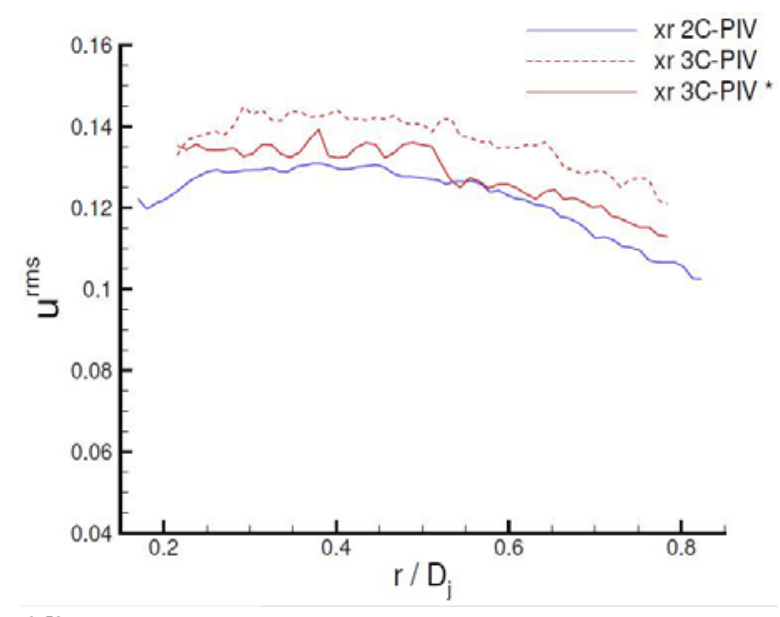

(d)

FIGURE 16 (Colour online) Radial profiles of axial RMS fluctuations $\mathrm{x} / \mathrm{D}_{\mathrm{j}}=$ (a) 1.64, (b) 4.0 , (c) 6.5 , (d) 10.0

\subsection{Spatio-Temporal Correlation Functions}

$2^{\text {nd }}$-order $R_{11}$ spatio-temporal plots in the $\mathrm{x}-\mathrm{r}$ plane were produced for all four measurement stations within the jet shear layer $\left(r / D_{j}=0.5\right)$; emphasis has been placed on shear layer locations, since examination of measurements on the jet centreline showed much better agreement between 3C-PIV and 3C-PIV* results (see Pokora (2009)). Comparisons between 2C-PIV, 3C-PIV as well as filtered 3C-PIV* data are shown in Figure 17 for 3 values of streamwise separation: $\eta_{1}=0.0 D_{j}, 0.2 D_{j}, 0.4 D_{j}$. The time axis is nondimensionalised by a characteristic time scale of the mean flow based on jet nozzle exit diameter and velocity. Whilst the 3C-PIV data shows significant discrepancies from the 2C-PIV data, the agreement between 2C-PIV and 3C-PIV* curves is much improved. The peak amplitude decay rate and the peak location shifts indicate the eddy convection velocity is in good agreement - in fact this is true for both 3C-PIV data sets. There is, as expected, a large underestimate of the peak correlation magnitude value in the original $3 \mathrm{C}$-PIV measurements. The close fit of $2 \mathrm{C}-\mathrm{PIV}$ and $3 \mathrm{C}-\mathrm{PIV}^{*}$ correlation functions shows that the stereoscopic technique, with the addition of the POD filter/correction procedure, can produce correlation function curves as accurate as the 2C-PIV data, but extends the possible measurement capability to all 3 velocity components. On the basis of this evidence, this filtering methodology has been used for all further 3C- PIV* data presented. 


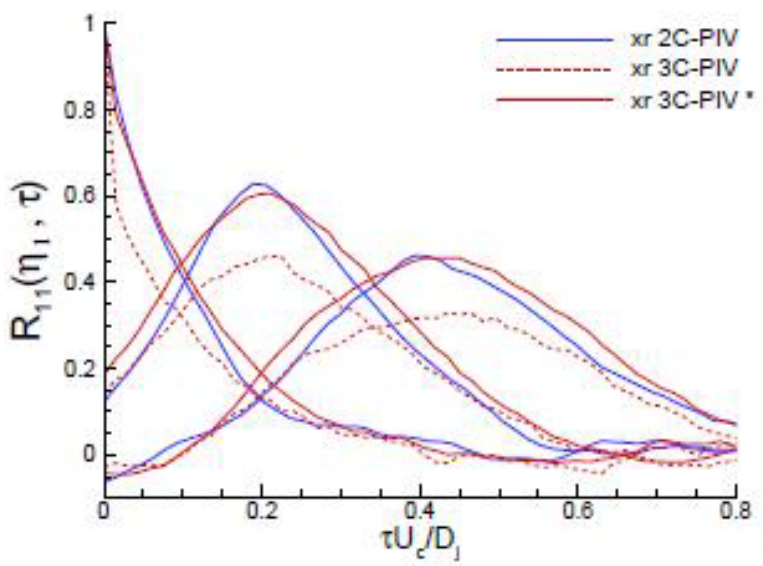

(a)

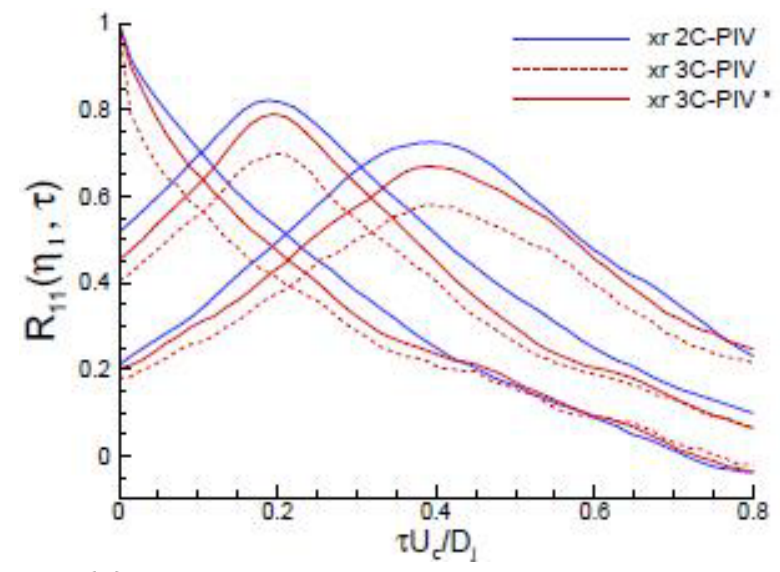

(c)

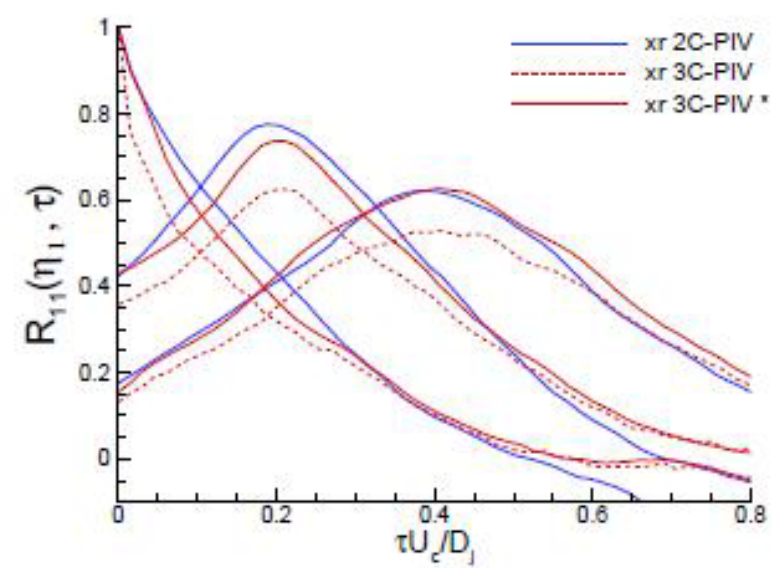

(b)

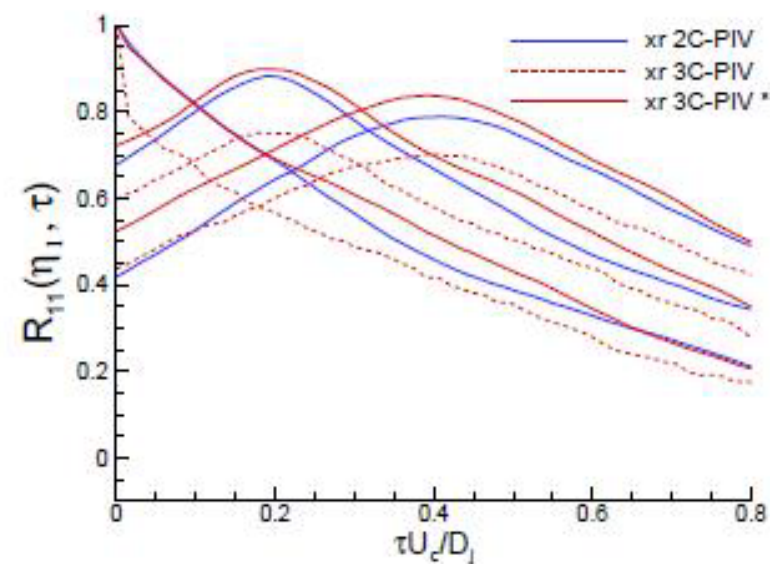

(d)

FIGURE 17 (Colour online) $R_{11}$ plots with axial separation - 2C-PIV, 3C-PIV and 3C-PIV* results $\mathrm{x} / \mathrm{D}_{\mathrm{j}}=$ (a) 1.64, (b) 4.0, (c) 6.5, (d) 10.0

To assess the quantitative accuracy of the current PIV measurements for 2-point quantities, $2^{\text {nd }}$ and $4^{\text {th }}$-order space-time correlation curves for streamwise, radial and circumferential separations were constructed. This was done principally for the location $\left(4 \mathrm{D}_{\mathrm{j}}, 0.5 \mathrm{D}_{\mathrm{j}}, 0^{0}\right)$ in the nozzle lip-line shear layer, which coincides with the measurement location chosen in Harper-Bourne's (2003) HWA data (labelled as CTA in the figures below). This data set was chosen as it includes not only $R_{11}$ but also $R_{11,11}$ measurements for all 3 separation vector components. Note that for radial and azimuthal separation correlations, a constant axial separation of $0.1 \mathrm{D}_{j}$ (radial) or $0.05 \mathrm{D}_{\mathrm{j}}$ (azimuthal) between the two points had to be included in the HWA study to avoid probe interference. This separation was also used in the 3C-PIV* radial evaluation to provide a direct comparison, but it was not possible to do so for the PIV azimuthal correlation measurement. In addition to this study, a location at the narrowest point in the shear layer measured $\left(1.5 D_{j}, 0.5 D_{j}, 0^{0}\right)$ was chosen to observe FoV size effects at the most challenging location, as well as comparison to other HWA and PIV measurements available. Results are given in figures 18-21; in all figures the horizontal axis has been evaluated using a constant convection velocity $U_{c}=0.6 U_{j}$ - this allows differences between the actual convection velocities in different data sets to be seen more readily.

Figure 18, showing data taken close to nozzle exit, provides conclusive evidence that 
the similarity of turbulence information between water and airflow experiments also
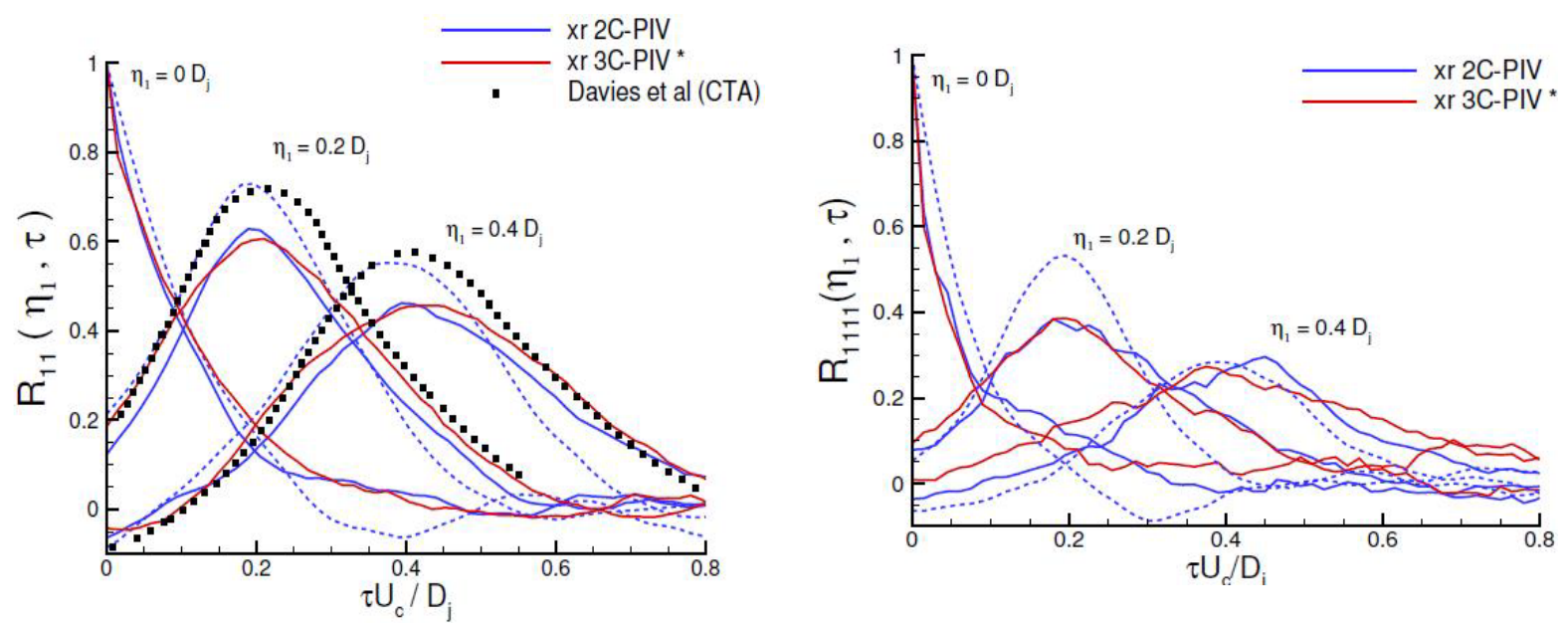

(a)

(b)

FIGURE 18 (Colour online) $R_{11}$ and $R_{11,11}$ plots for axial separations at $x / \mathrm{D}_{\mathrm{j}}=1.5, \mathrm{r} / \mathrm{D}_{\mathrm{j}}=0.5$

Solid lines - 25mmx25mm FoV, dashed lines - 40mmx40mm FoV

extends to the correlation functions. For $R_{11}$ direct comparison is made with HWA data (Davies et al. (1962), labelled CTA) as well as with 2C-PIV (two FoV sizes) and 3C$\mathrm{PIV}^{*}$ results. It is at this location where the shear layer is very thin that the PIV FoV size is most important. Figure 18a shows that the larger FoV (which contains more sub-cell filtering) gives higher peak amplitudes, but this is certainly a fortuitous and erroneous result. The agreement between the eddy convection velocities from the various experiments is seen to be good by the match in peak correlation locations at all separation values. The $2 \mathrm{C}$ - and $3 \mathrm{C}_{-} \mathrm{PIV}^{*}$ data are in very good agreement with each other and with the HWA data (although the PIV data show reduced peak amplitude with separation). The decrease in peak value with separation distance indicates the large scale mixing causes a 'non-frozen' nature of the shear layer turbulence, and the growth in correlation function width with increasing $\eta_{1}$ shows the growth in eddy size. There are no other measurements to compare with the $4^{\text {th }}$-order correlation at this location. The $4^{\text {th }}$-order function displays a more rapid decay in both space and time, noted by Harper-Bourne (2003) as being consistent with the greater frequency content relative to $2^{\text {nd }}$-order quantities. The agreement between the two $25 \mathrm{~mm}$ FoV results (2C-PIV, 3C-PIV*) is very good for $R_{11,11}$.

Figure 19 presents similar $R_{11}$ and $R_{11,11}$ functions with axial separation, but at $\mathrm{x} / \mathrm{D}_{\mathrm{j}}=$ 4.0. Comparison with HWA results for $R_{11}$ is of similar quality as at the upstream station. The combined LDA/PIV method of Chatellier and Fitzpatrick (2005) shows considerably inferior performance compared to the PIV-only technique. Close inspection of PIV and HWA data shows a slightly higher eddy convection velocity for PIV. The HWA peak amplitudes for $R_{11}$ are again greater than with PIV, but this difference is in fact reduced for $R_{11,11}$, where PIV/HWA agreement is very good at all axial separations. 


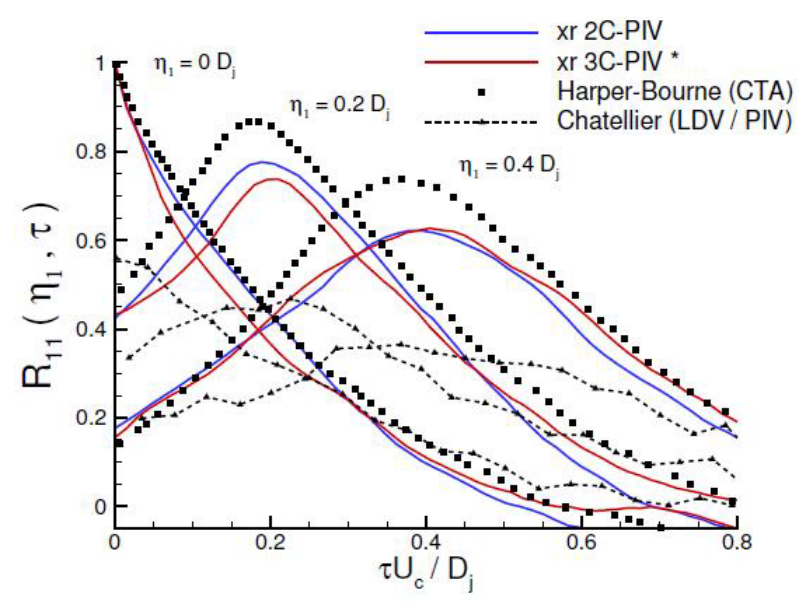

(a)

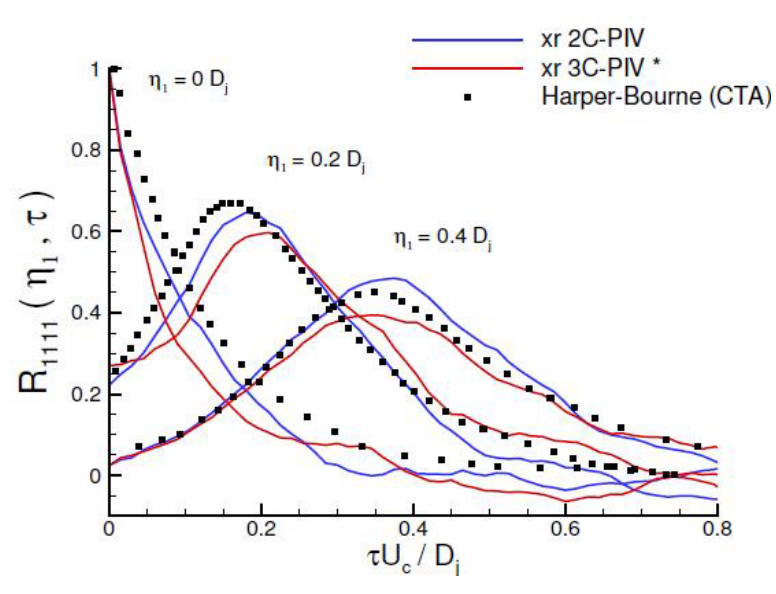

(b)

FIGURE 19 (Colour online) $R_{11}$ and $R_{11,11}$ plots for axial separations at $/ \mathrm{D}_{\mathrm{j}}=4.0, \mathrm{r} / \mathrm{D}_{\mathrm{j}}=0.5$

The effect of radial separation is illustrated in figure 20. For $R_{11}$ the time to the peak location decreases as $\eta_{2}$ increases, the shifted point moving radially inwards across the shear layer indicating a phase shift. For $R_{11,11}$ agreement is very good, with the peak occurring close to zero time for small $\eta_{3}$ separations as observed in the HWA data. The PIV/HWA agreement for the $4^{\text {th }}$-order components is not quite as good as for $2^{\text {nd }}$ order quantities. Note the fact that the peak values at zero $\eta_{2}$ are not unity is caused by the fact that an axial separation of $0.1 \mathrm{D}_{\mathrm{j}}$ has had to be included in evaluating $R_{11}$ and $R_{11,11}$ to match the HWA configuration as noted above; thus the $\eta_{2}=0 \mathrm{D}_{\mathrm{j}}$ correlation is the same as the correlation for $\eta_{1}=0.1 \mathrm{D}_{\mathrm{j}}$.

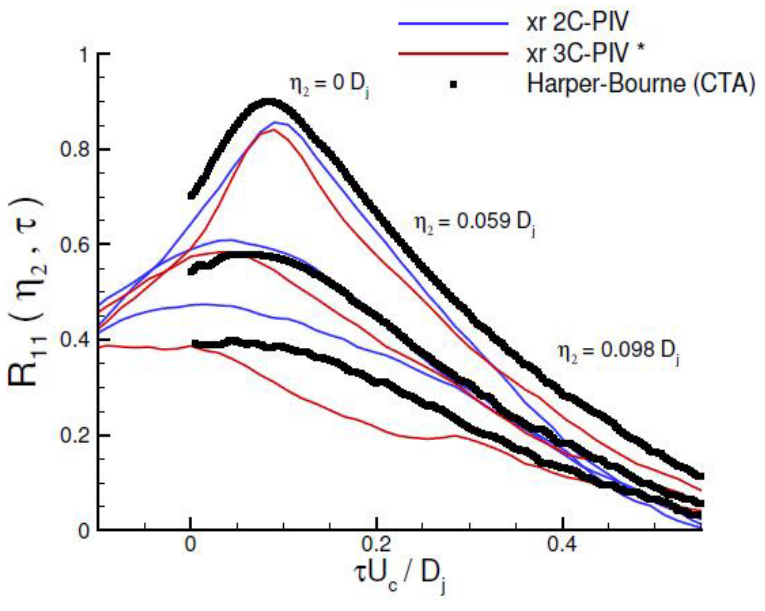

(a)

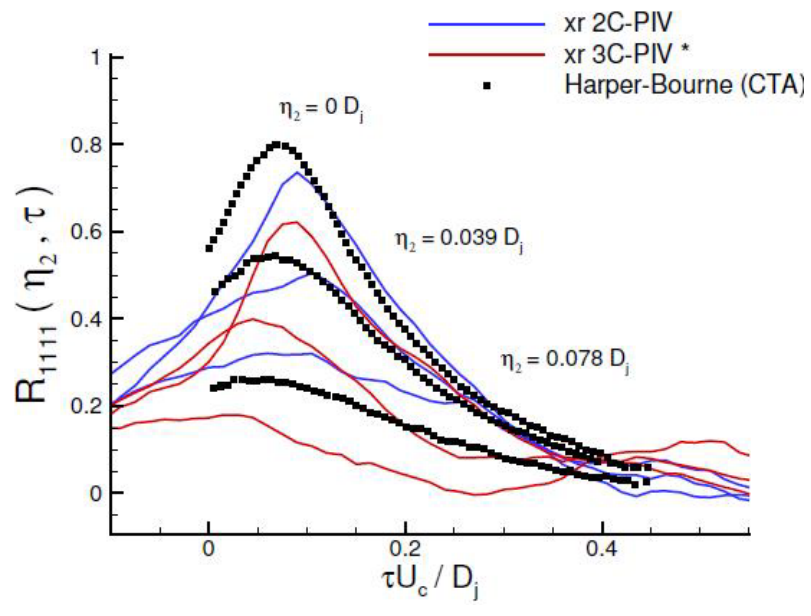

(b)

FIGURE 20 (Colour online) $R_{11}$ and $R_{11,11}$ plots for radial separations at $/ \mathrm{D}_{\mathrm{j}}=4.0, \mathrm{r} / \mathrm{D}_{\mathrm{j}}=0.5$

The effect of azimuthal separation is presented in figure 21; for $R_{11}\left(\eta_{3}, \tau\right)$ the alignment of HWA and PIV data is very good; this is also the case for $R_{11,11}\left(\eta_{3}, \tau\right)$, although the zero comparison shows greatest discrepancy - this is probably to a large extent caused by the neglect of the small axial separation used in the HWA data in the PIV measurement. These azimuthal correlations are significantly flatter than the axial or radial for quite 
small increases in the separation vector; this is consistent with the axisymmetric nature of the jet flow.

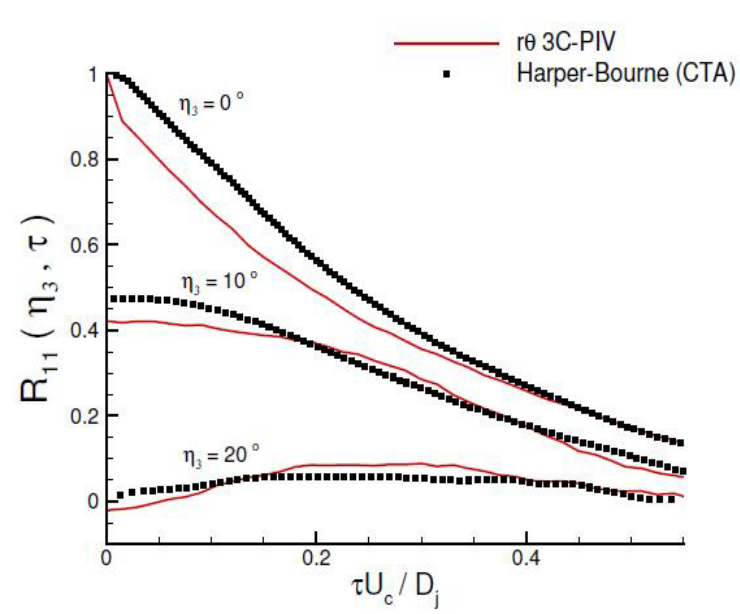

(a)

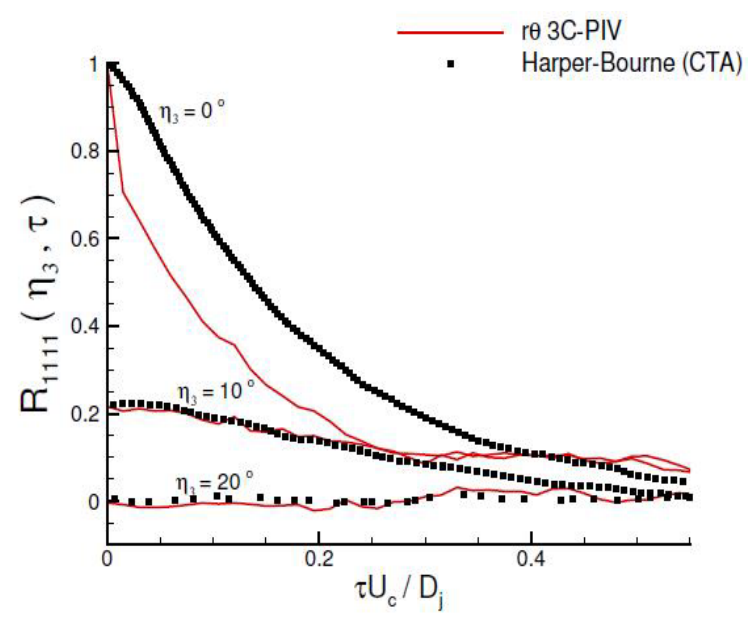

(b)

FIGURE 21 (Colour online) $R_{11}$ and $R_{11,11}$ plots for azimuthal separations at $/ \mathrm{D}_{\mathrm{j}}=4.0, \mathrm{r} / \mathrm{D}_{\mathrm{j}}=0.5$

Since this is perhaps the first time that PIV and HWA measurements for spatiotemporal correlations have been directly and comprehensively compared (certainly the

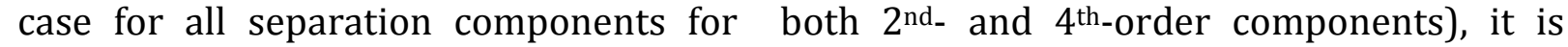
worthwhile providing a summary overview of the PIV performance. In general both techniques have advantages and disadvantages. Both methods contain error sources problematic for measuring the quantities of interest here. HWA has high temporal resolution, but use of anti-aliasing techniques can lead to increased correlation peak magnitudes. Planar optical measurement techniques such as PIV more conveniently allow the visualisation of correlation functions for components other than the axial velocity and for larger ranges of the separation vector than probe-based techniques. However, PIV is subject to sub-cell filtering and spurious vector instrumentation noise, which can cause reduced correlation peak magnitudes. As illustrated in the present study, these can be controlled using smaller FoVs, although this then places some limitations on the extent of separation vector possible. Nevertheless, it is believed that the correct quantitative behaviour of the current PIV data has been adequately demonstrated by the above HWA/PIV comparisons. To balance the slight fall-off in accuracy, PIV offers the opportunity to measure more components of $R_{i j}$ and $R_{i j, k l}$, it is this potential which was exploited next in the current study.

Existing data on 2-point correlation maps for jet flows has concentrated on axial separation distance/time delay maps. Figure 22 shows such maps extracted from the present $3 \mathrm{C}-\mathrm{PIV}^{*}$ results for the point $\left(4 \mathrm{D}_{\mathrm{j}}, 0.5 \mathrm{D}_{\mathrm{j}}, 0^{0}\right)$. Because of the quality of the current measurements, maps for both $2^{\text {nd }}-$ and $4^{\text {th }}$ - order components can be included. The diagonal trajectory of the peak correlation value allows the eddy convection velocity to be estimated; figure 22 shows that this is essentially the same $\left(0.6 \mathrm{U}_{\mathrm{j}}\right)$ for all correlation components shown, and for both $2^{\text {nd }}$ - and $4^{\text {th }}$-order quantities. 


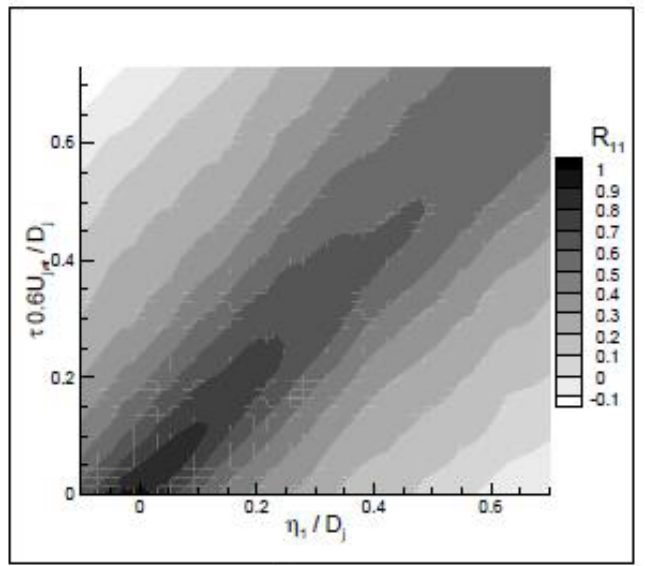

(a) $R_{11}$

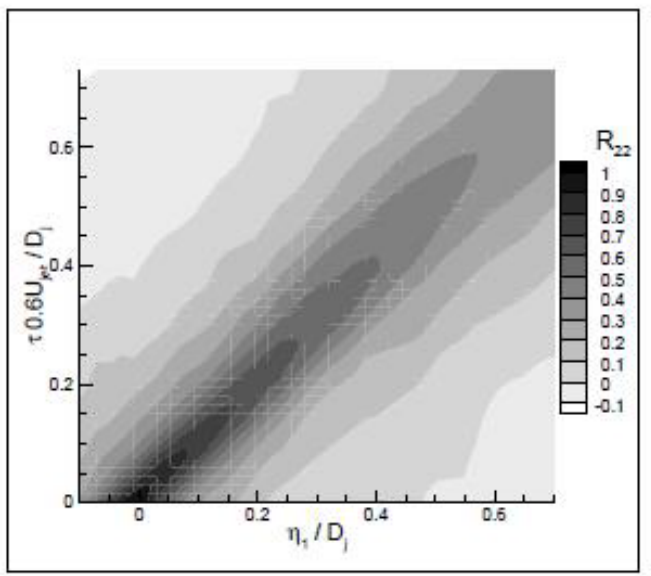

(c) $R_{22}$

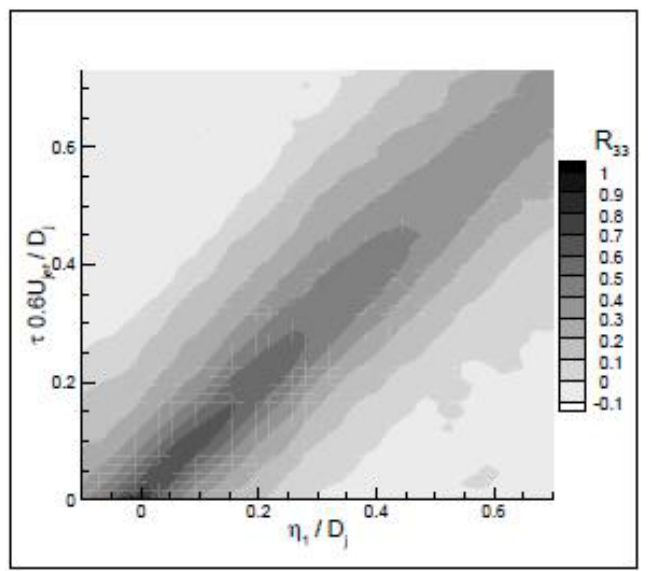

(e) $R_{33}$

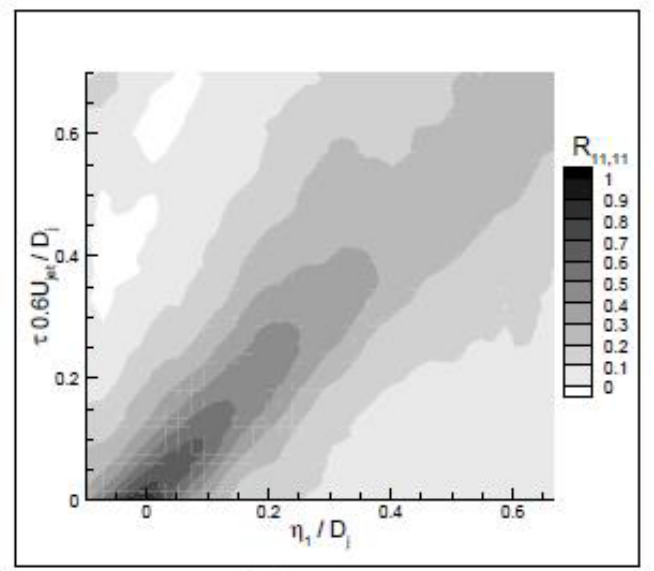

(b) $R_{11,11}$

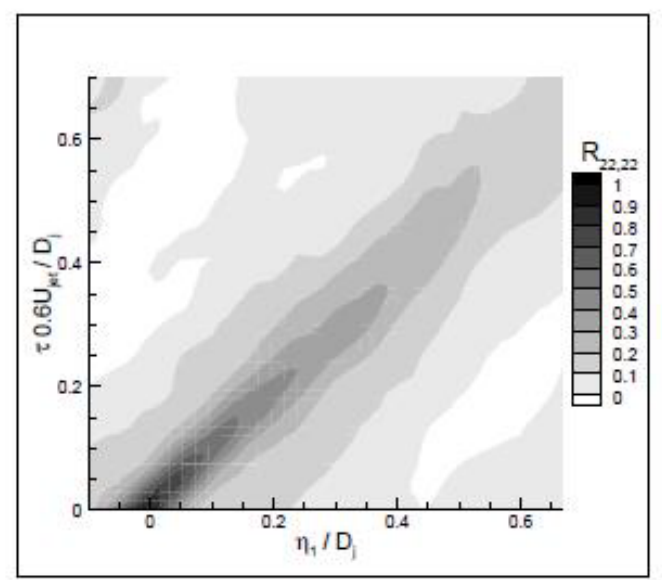

(d) $R_{22,22}$

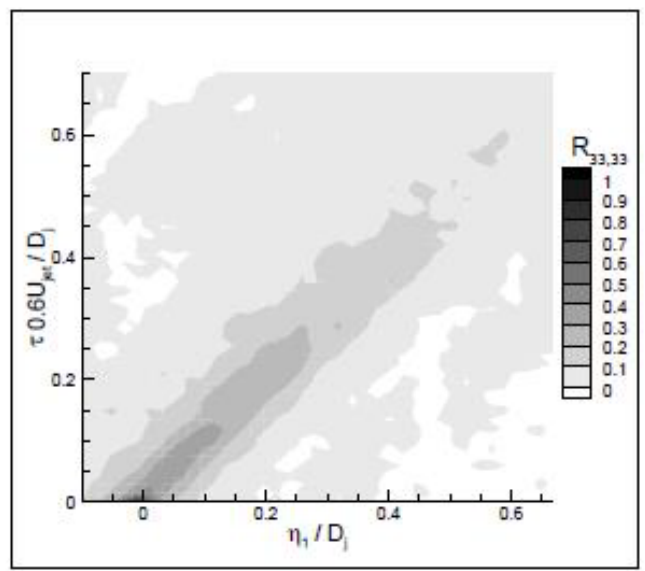

(f) $R_{33,33}$

FIGURE $222^{\text {nd }}$ and $4^{\text {th }}$ rank tensor components - spatio-temporal correlation maps at $\left(4 \mathrm{D}_{\mathrm{j}}, 0.5 \mathrm{D}_{\mathrm{j}}, 0^{0}\right)$

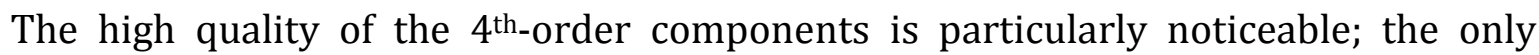

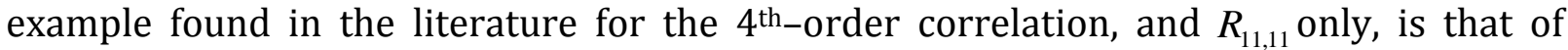
Bridges and Wernet (2003), where the trajectory was difficult to discern because of the 
extremely high noise level. The $4^{\text {th }}$-order maps are narrower than the $2^{\text {nd }}$-order as expected from the faster decay rates noted above; for both $R_{i j}$ and $R_{i j, k l}$ the axial component displays larger regions of high correlation extending further along the trajectory compared to radial and azimuthal components. Maps such as these allow evaluation of Lagrangian-evaluated quantities, often used in aeroacoustic models based on the use of a frame of reference moving with the eddies as suggested by Lighthill (1952, 1954). For example the Lagrangian length scale ${ }^{k} L_{i j}^{L}$, is defined as the spatial separation at which the correlation decays to 1 /e of its initial value in a moving frame of reference (i.e. along the eddy convection velocity axis). This quantity has been evaluated from the correlation maps shown in figure 22 and may be compared with HWA data from HarperBourne (2003) for both $2^{\text {nd }}$ - and $4^{\text {th }}$ - order Lagrangian length scales. For axial velocity and all 3 directions this comparison is shown in tables 4 and 5:

\begin{tabular}{|c|c|c|c|}
\hline & ${ }^{1} L_{11}^{L} / \mathrm{D}$ & ${ }^{2} L_{11}^{L} / \mathrm{D}_{\mathrm{i}}$ & ${ }^{3} L_{11}^{L} / \mathrm{D}$ \\
\hline $\begin{array}{c}\text { Harper-Bourne (2003) } \\
\text { HWA data }\end{array}$ & 1.14 & 0.114 & 0.101 \\
\hline Current PIV data & 1.15 & 0.118 & 0.099 \\
\hline \multicolumn{4}{|c|}{ TABLE 4 Comparison of $2^{\text {nd }}$-order Lagrangian length scales of axial velocity } \\
\hline & & ${ }^{2} L_{11}^{L}$ & ${ }^{3} L_{11}^{L}$ \\
\hline Harper-Bourne (2003) & 0.515 & 0.071 & 0.073 \\
\hline HWA data & & & \\
\hline Current PIV data & 0.426 & 0.058 & 0.066 \\
\hline
\end{tabular}

The comparison between HWA and PIV results is excellent; the anisotropy of the length scales is reproduced very well, with an order of magnitude difference between axial and transverse components at $2^{\text {nd }}$ - order, with a similar but somewhat smaller factor at $4^{\text {th }}$ order.

An alternative presentation of the data is to plot correlation maps in physical space as done by Fleury et al. (2008), although for $\tau=0$ only. Figures 23 and 24 display $2^{\text {nd }}$ - and $4^{\text {th }}$-order correlation maps in the $\mathrm{x}-\mathrm{r}$ plane, with the present investigation extending the data set to various time delays and all 3 velocity components. For the $2^{\text {nd }}$-order correlations (figure 23) the elongated elliptical shape of the axial component compared to the more circular shape of the radial component is clear to see, and this has also been observed in Fleury et al. (2008). The circular shape tends towards elliptic at larger delay times. Figure 23 extends this information to include the $R_{33}$ component; this is initially similar to $R_{22}$ although narrower, but becomes elliptical more quickly than $R_{22}$ as delay time increases. The close to axial trajectory of the peak value for all 3 components is very noticeable. A visual assessment of the quality (low spurious noise levels) of the PIV correlation maps shows well defined edges to the correlation contours and a constant zero background correlation level, indicating low noise levels and high quality. 


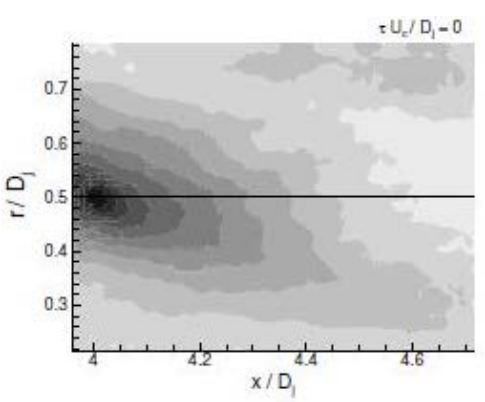

(a) $R_{11}$

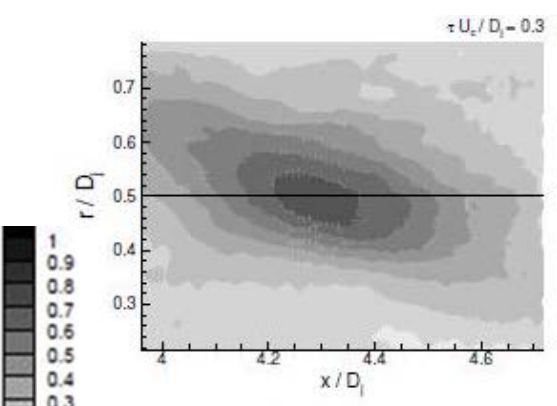

(b) $R_{11}$

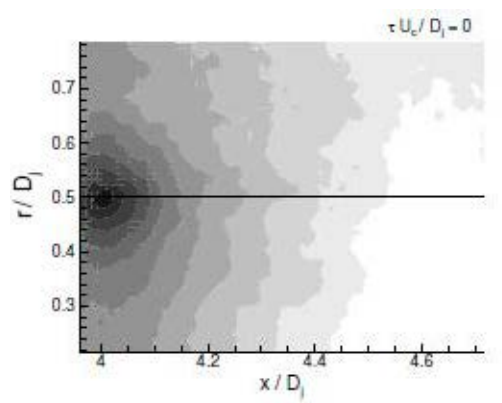

(d) $R_{22}$

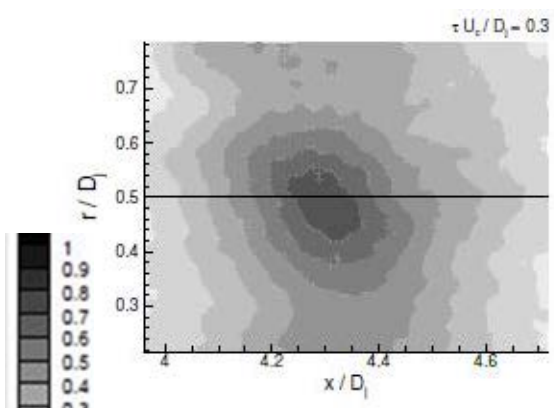

(e) $R_{22}$

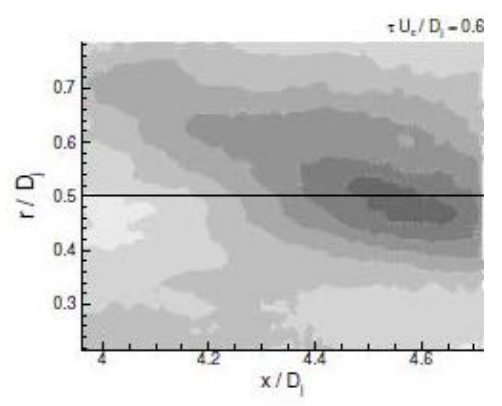

(c) $R_{11}$

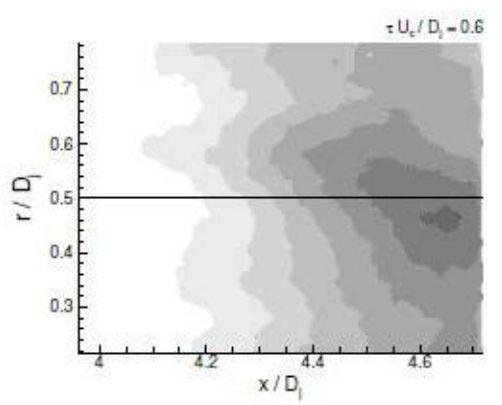

(f) $R_{22}$

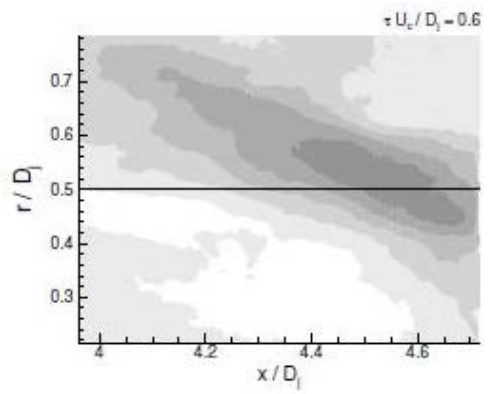

(i) $R_{33}$

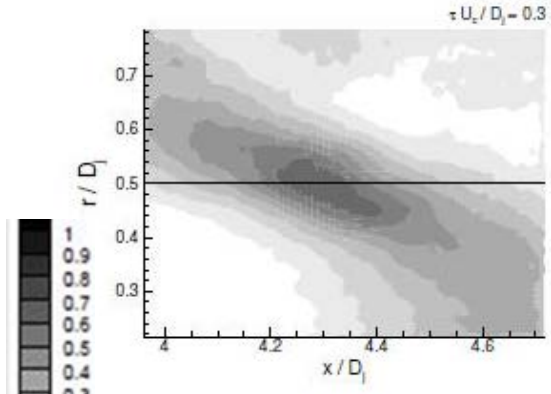

(h) $R_{33}$ (g) $R_{33}$

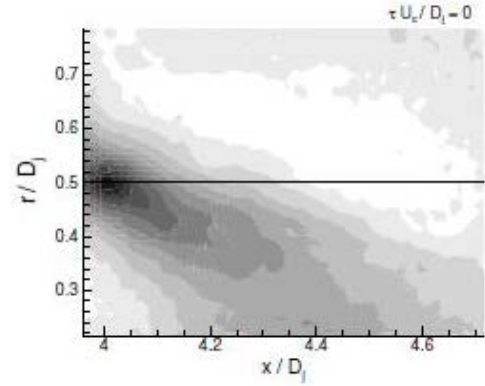

FIGURE $232^{\text {nd }}$-order spatio-temporal correlations at $\left(4 \mathrm{D}_{\mathrm{j}}, 0.5 \mathrm{D}_{\mathrm{j}}, 0^{0}\right)$

This low noise level trend continues when examining similar plots for the $4^{\text {th }}$-order correlations of axial fluctuation with axial separation (i.e. $R_{11,11}\left(\underline{x}, \eta_{1}, \tau\right)$ ) as shown in figure 24 . The more compact nature of the higher order correlations is very well illustrated in these plots. Once again it is noteworthy that low noise levels are retained for the $4^{\text {th }}$-order correlation, although faster deterioration is visible compared to the $2^{\text {nd }}$ - order plots in figure 23 . 


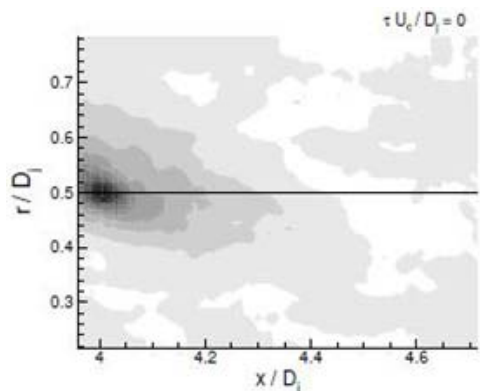

(a) $R_{1111}$

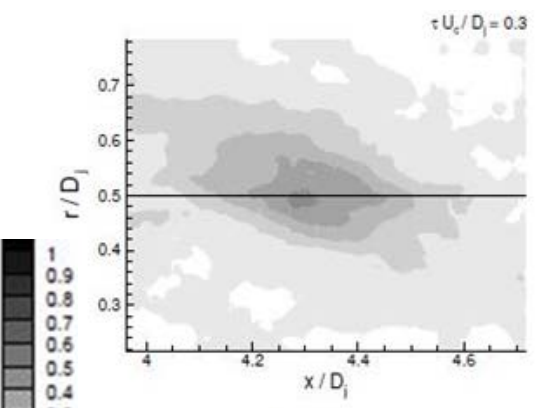

(b) $R_{1111}$

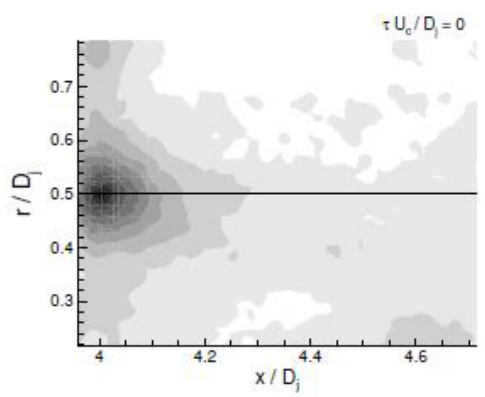

(d) $R_{2222}$

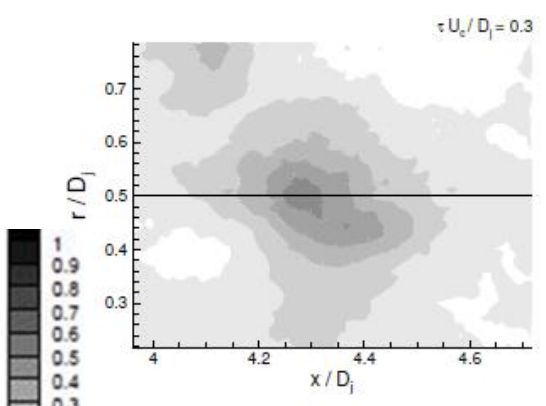

(e) $R_{2222}$

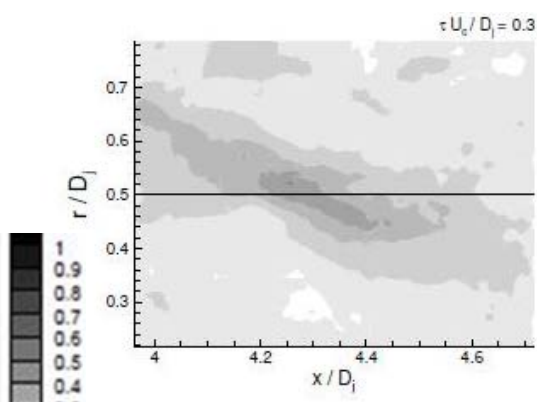

(h) $R_{3333}$

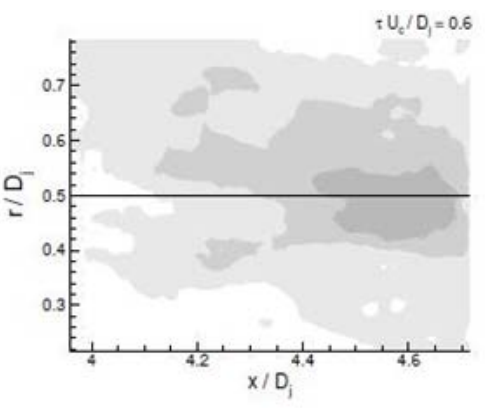

(c) $R_{1111}$

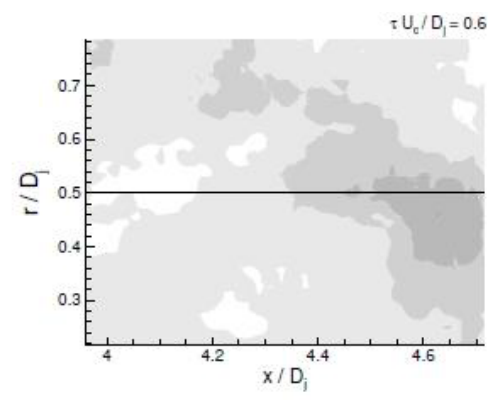

(f) $R_{2222}$

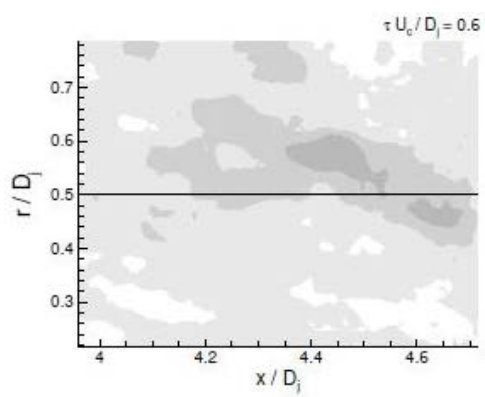

(i) $R_{3333}$

FIGURE $244^{\text {th }}$-order spatio-temporal correlations at $\left(4 \mathrm{D}_{\mathrm{j}}, 0.5 \mathrm{D}_{\mathrm{j}}, 0^{0}\right)$

The availability of measurements to guide aeroacoustic modelling has until recently been restricted to the axial component only. Models have therefore generally made the assumption that all components of $R_{i j, k l}$ may be related to $R_{11,11}$ by simple constant ratios. The present data cover enough components of $R_{i j, k l}$ to allow this assumption to be examined. Although this $4^{\text {th }}$ rank tensor contains a total of 81 components, the symmetry between tensor indices reduces the number of independent components to 36 and if emphasis is placed on the peak amplitudes at zero space and time separations, then this number reduces further to 21 . The present measurements allow the relative peak 
amplitudes at zero values of $(\underline{\eta}, \tau)$ for all $62^{\text {nd }}-$ order (relative to $R_{11}$ ) and all $214^{\text {th }}$-order (relative to $R_{11,11}$ ) independent components to be examined. Once again it is useful to start by comparing the PIV-measured data with what is available from HWA measurements. Morris and Zaman (2010) have used cross-wire HWA measurements to evaluate 3 components of $R_{i j, k l}$ in the shear layer and along the jet centreline. Comparison is provided in figure 25 between this data and the current PIV results for the region just downstream of the end of the potential core, with generally good agreement. Another assumption made in aeroacoustic modelling is that the relative amplitudes are independent of streamwise position. Figure 25 shows this is not true for all components in this comparison, particularly for the $(11,22)$ component. Since the local flow structure alters from that of an annular shear layer to that of a self-similar jet beginning at the potential core end, it is perhaps not so surprising that these ratios vary with $\mathrm{x}$.

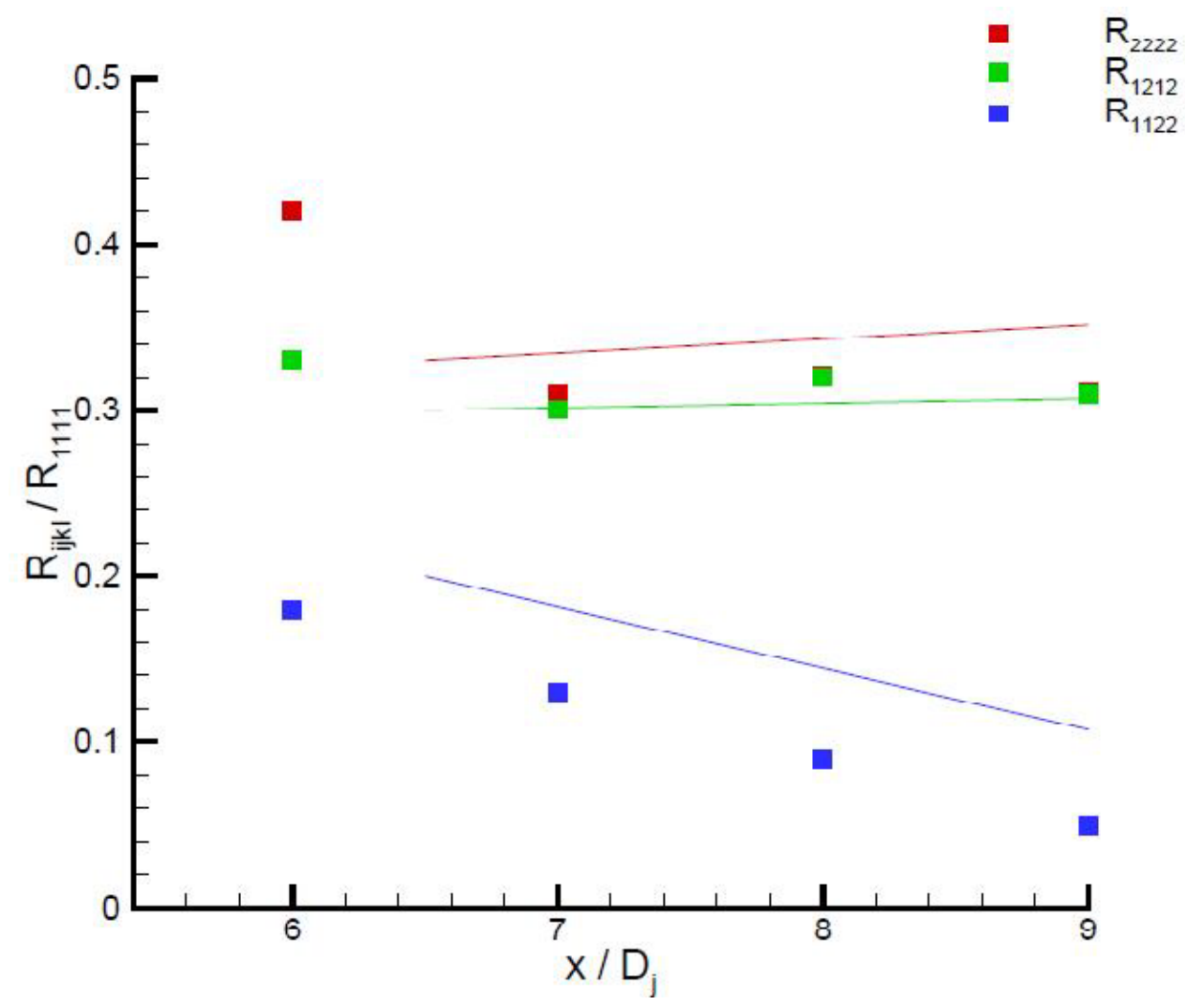

FIGURE 25 (colour online) Comparison of PIV data (symbols) with HWA data Morris \& Zaman (2010) (lines) for $4^{\text {th }}$-order peak correlation relative amplitudes along jet centreline.

Figures 26 and 27 provide full coverage of all relevant $R_{i j}$ and $R_{i j, k l}$ relative peak amplitudes. For $R_{i j}$ (the point $\left(4 \mathrm{D}_{\mathrm{j}}, 0.5 \mathrm{D}_{\mathrm{j}}, 0^{0}\right)$ is shown) good comparison is obtained between different PIV measurements, and the agreement with HWA is reasonable; the ratios of $R_{11} R_{22}$ and $R_{33}$ reflect the anisotropy of turbulence in a shear layer. 


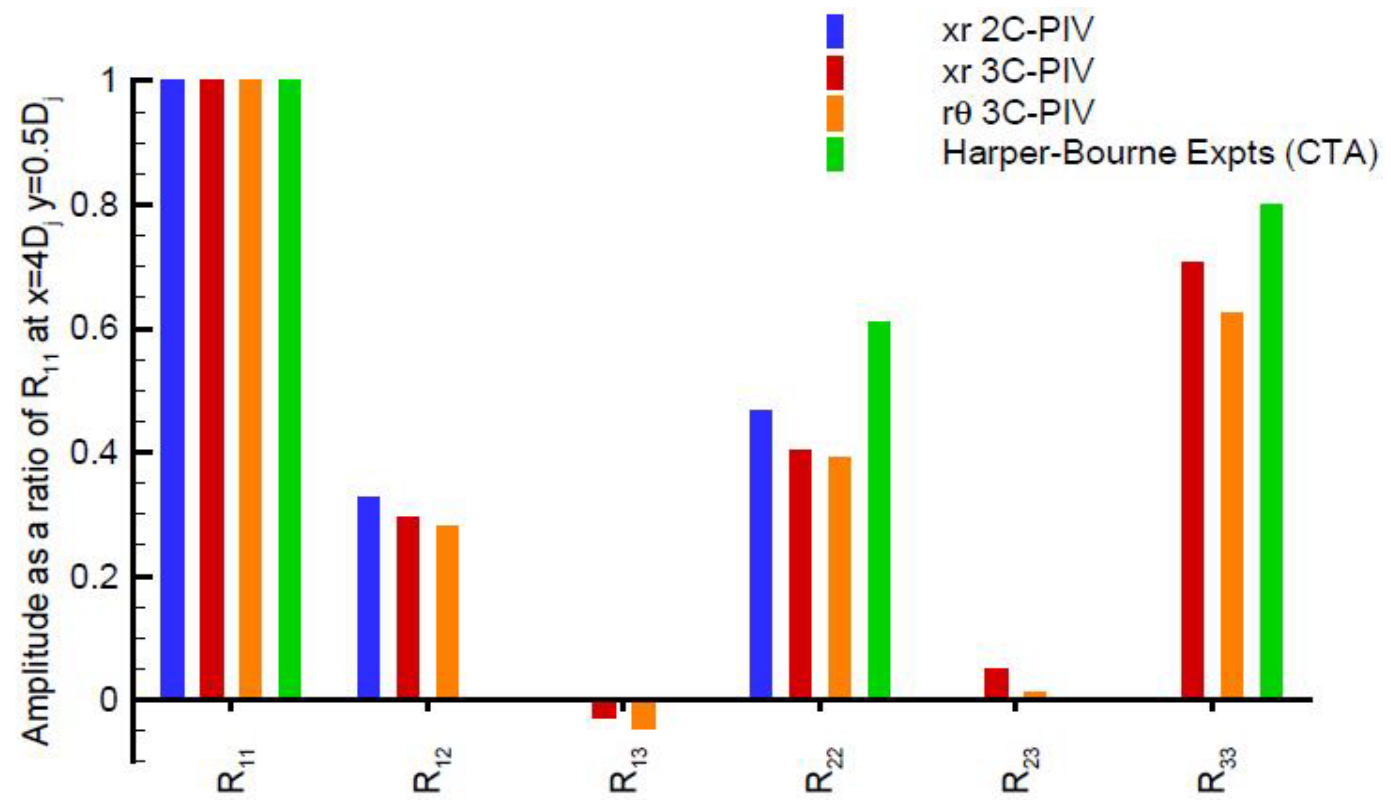

FIGURE 26 (colour online) Comparison of $2^{\text {nd }}$-order peak amplitudes relative to $R_{11}$ for PIV and HWA results

For $R_{i j, k l}$ data is presented at $\mathrm{x} / \mathrm{D}_{\mathrm{j}}=10.0$ but both shear layer $\left(\mathrm{r} / \mathrm{D}_{\mathrm{j}}=0.5\right)$ and centerline $\left(r / D_{j}=0.0\right)$ locations are now included. One significant result is that of the possible 21 magnitudes, in the important shear layer region only 6 are significant, with the rest smaller by comparison; these components are: $R_{11,11}, R_{11,12}, R_{12,12}, R_{13,13}, R_{22,22}$, and $R_{33,33}$. Such data is extremely useful for calibration of noise source models, as shown already by Karabasov et al. (2010).

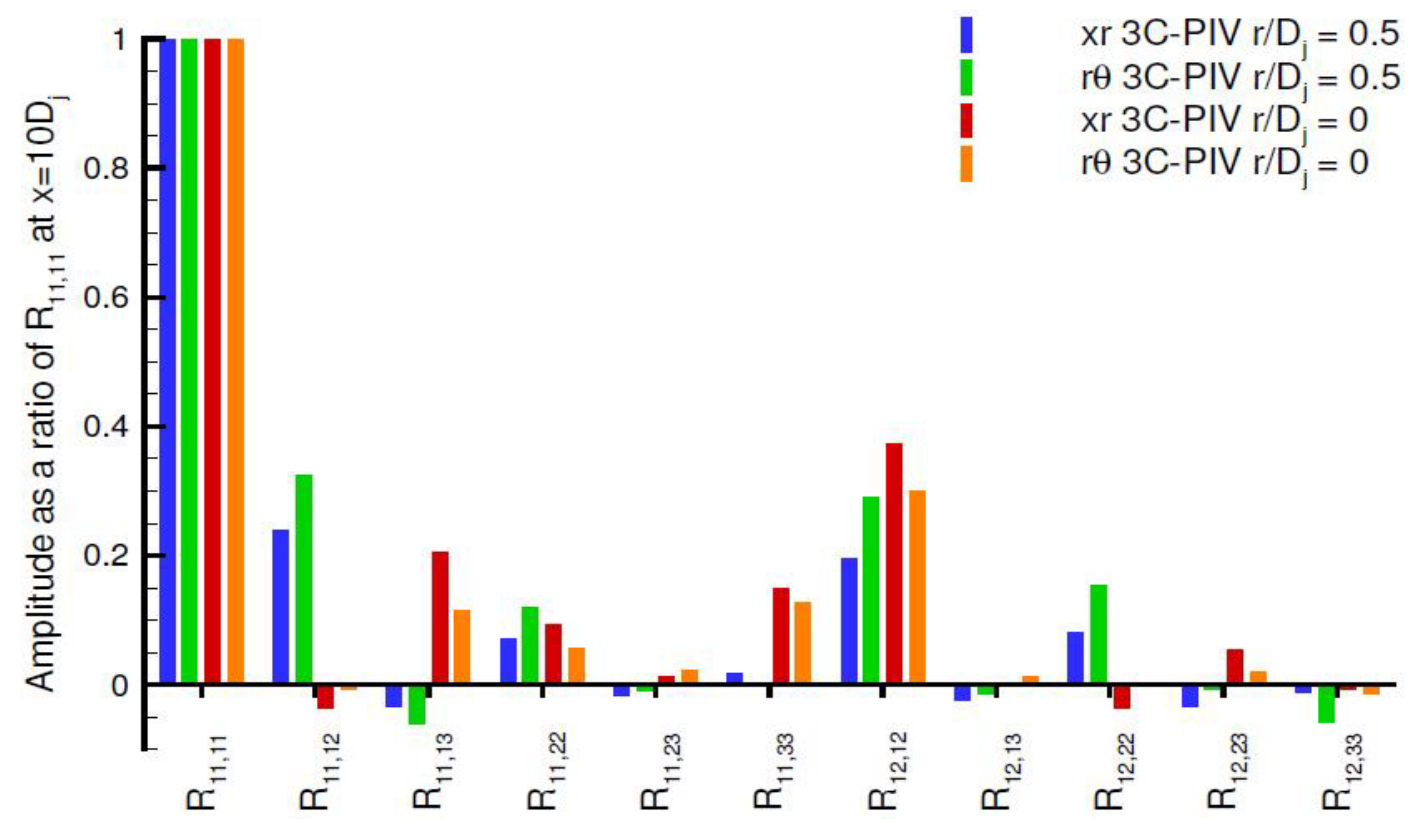




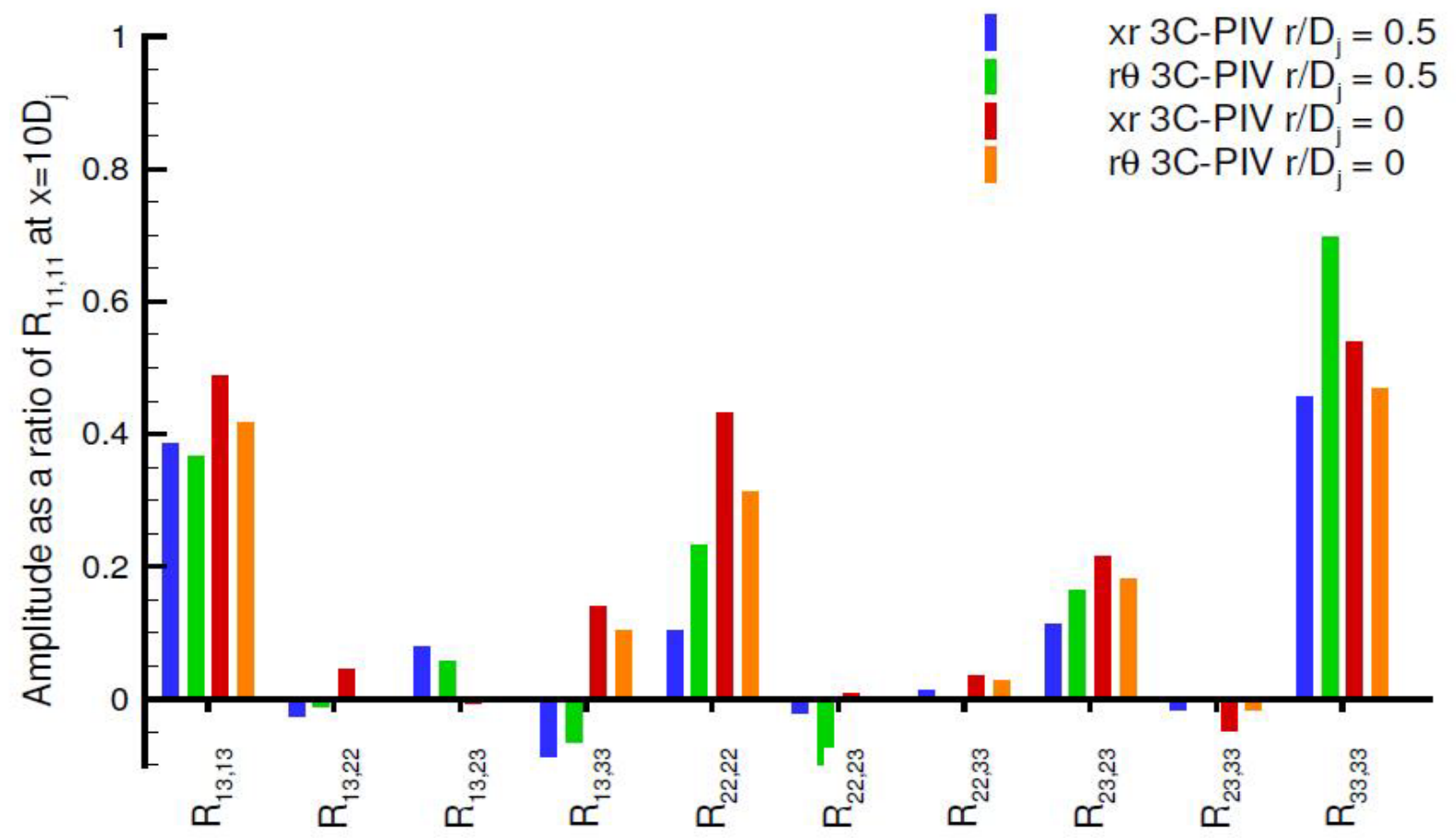

FIGURE 27 (colour online) Comparison of $4^{\text {th }}$-order amplitudes relative to $R_{11,11}$ for PIV results

As noted above a common assumption about the absolute correlation amplitudes is that the relative amplitudes are independent of axial position, and this was partially examined above for the jet centreline. Figure 28 shows the relative amplitude for some major $R_{i j, k l}$ components at several axial locations along the nozzle lipline.

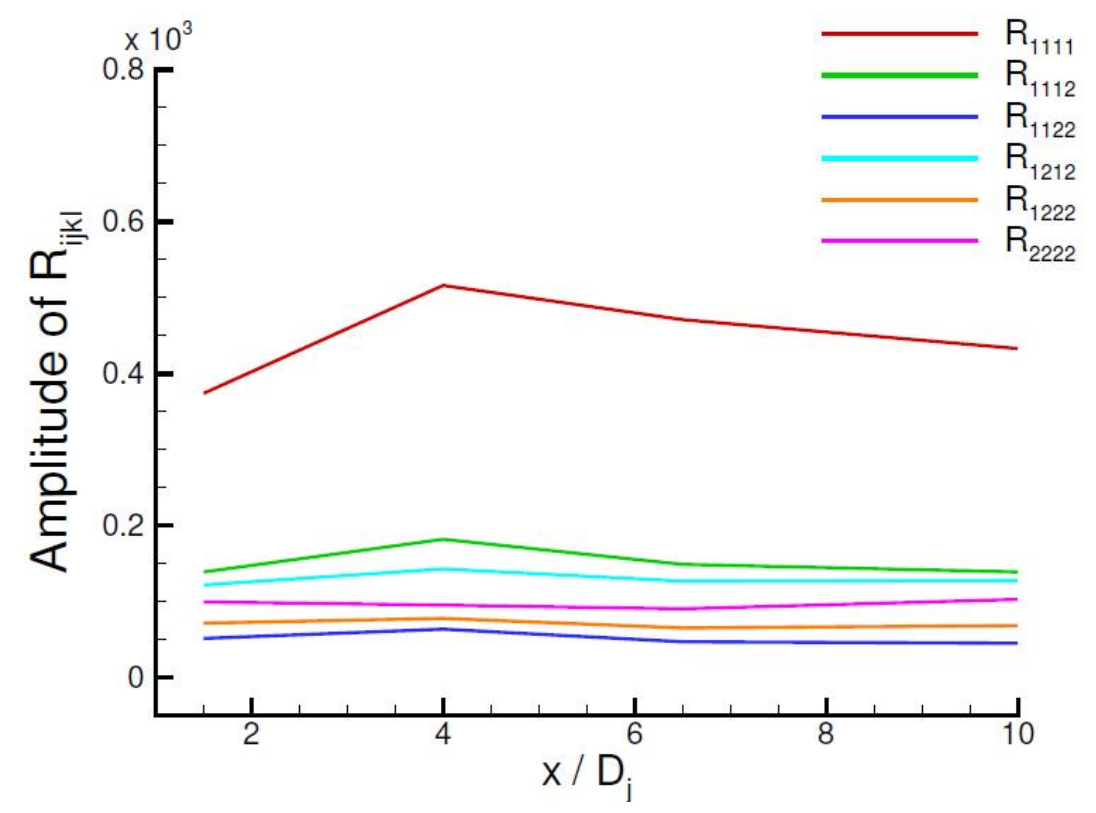

FIGURE 28 (colour online) Axial development of $4^{\text {th }}$-order correlation realtive amplitudes along shear layer

The assumption seems to be much more acceptable for shear layer locations, with all correlation components showing little variation with axial location from nozzle exit to the 
end of the potential core (more variation seen in $R_{11,11}$ but still reasonable). One possible reason for this is that, unlike the centreline region considered in figure 25 , once the local characteristics of the lipline flow have adjusted to the transition from a boundary layer to a shear layer, the self-similar nature of the flow means that the turbulent structures change little with downstream distance.

\subsection{Approximation of $4^{\text {th-order }}$ correlations via products of $2^{\text {nd }}$-order correlations}

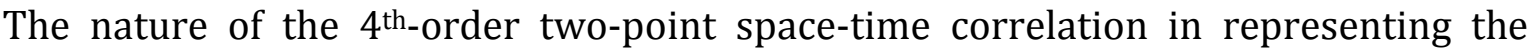
fundamental source of turbulence generated noise means that its modelling lies at the heart of conventional acoustic analogies. Since $R_{i j, k l}$ has until recently defied attempts to measure it directly, at least for the vast majority of its many components, then most

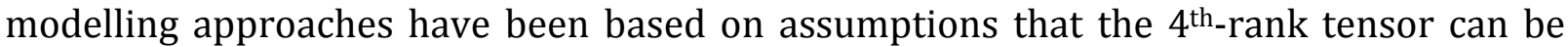
approximated in terms of $2^{\text {nd }}$-rank tensor expressions. Millionshchikov (1941) (see also Batchelor (1953)) was the first to propose a relationship between $4^{\text {th }}$-order and $2^{\text {nd }}$-order velocity correlations, based on an assumption of a 'quasi-Gaussian' (or quasi-normal) joint probability density function between velocity components. Based on this assumption, this yields:

$$
\overline{u_{i}^{\prime} u_{j}^{\prime} u_{k}^{\prime} u_{l}^{\prime}} \approx \overline{u_{i}^{\prime} u_{j}^{\prime}} \cdot \overline{u_{k}^{\prime} u_{l}^{\prime}}+\overline{u_{i}^{\prime} u_{k}^{\prime}} \cdot \overline{u_{j}^{\prime} u_{l}^{\prime}}+\overline{u_{i}^{\prime} u_{l}^{\prime}} \overline{\cdot u_{j}^{\prime} u_{k}^{\prime}}
$$

Whilst there is evidence in support of the quasi-normal approximation for single point correlations (although mainly in simple turbulent flow such as grid turbulence), its extension to two-point statistics is less easy to justify; Morris and Zaman (2010) argue that its extension to jet shear flow turbulence is problematic. Bridges and Wernet (2003) have attempted to use their direct measurements of $R_{11,11}$ and $R_{22,22}$ to assess the correctness of the quasi-normal approximation, but, as noted above, their $4^{\text {th }}$-order measurements suffered so badly from noise and scatter that drawing firm conclusions from this comparison was virtually impossible. The high quality and comprehensive nature of the present measurements encouraged an exploration of the quality of the quasi-normal approximation using the current data.

Thus, using the definition of $R_{i j, k l}$ given above in eqn. (1.3), the quasi-Gaussian approximation to $R_{i j, k l}$ may be written as follows:

$$
R_{i j, k l}^{Q G}(\underline{x}, \underline{\eta}, \tau)=\frac{\overline{u_{i}^{\prime}(A) u_{k}^{\prime}(B)} \overline{\cdot u_{j}^{\prime}(A) u_{l}^{\prime}(B)}+\overline{u_{i}^{\prime}(A) u_{l}^{\prime}(B)} \overline{\cdot u_{j}^{\prime}(A) u_{k}^{\prime}(B)}}{\left[\overline{u_{i}^{\prime 4}}(A) \cdot \overline{u_{j}^{\prime 4}}(A) \cdot \overline{u_{k}^{\prime 4}}(B) \cdot \overline{u_{l}^{\prime 4}}(B)\right]^{1 / 4}-\left[\overline{u_{i}^{\prime 2}}(A) \cdot \overline{u_{j}^{\prime 2}}(A) \cdot \overline{u_{k}^{\prime 2}}(B) \cdot \overline{u_{k}^{\prime 2}}(B)\right]^{1 / 2}}
$$

Note that that the PIV-measured quantities have been retained in the denominator used to non-dimensionalise this expression so that inconsistencies in the quasi-Gaussian approximation may be more readily revealed.

Figure 29 first examines the validity of the quasi-normal approximation for the principle component $R_{11,11}$ and 4 points in the jet shear layer. The overlay between the PIVmeasured $R_{11,11}$ curves with varying axial separation and time delay and the $R_{11,11}^{Q G}$ approximation is in general reasonable, but non-negligible discrepancies are revealed. The quasi-normal approximation fails to pick up any negative loops in the correlation, and with increasing axial separation, an over-estimation in the magnitude is observed. The 
approximated correlations also display values at zero separation above unity, which is a further indication of error.

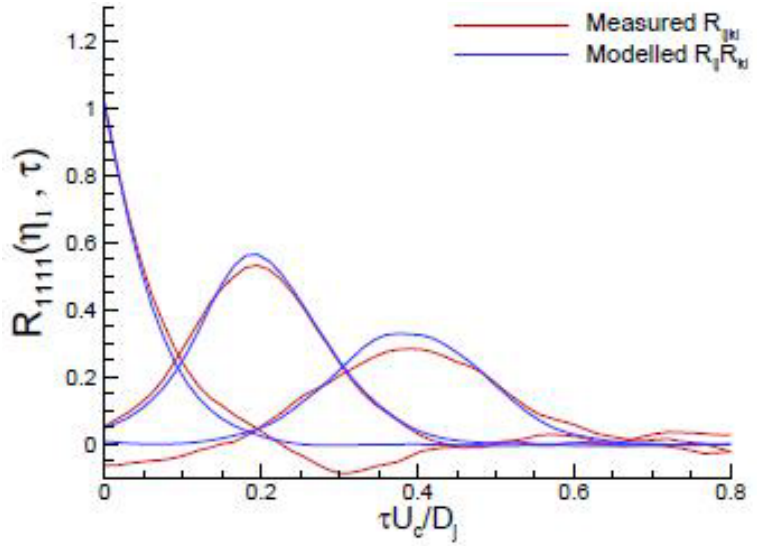

(a) $x / D_{j}=1.5$

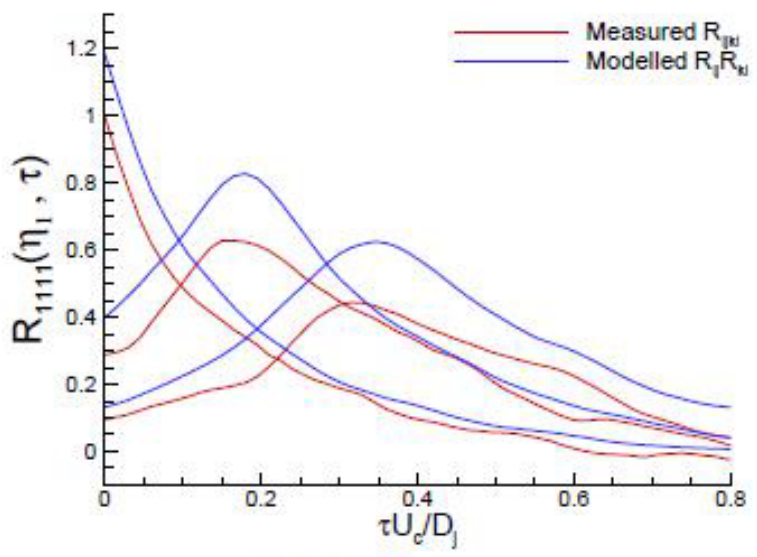

(c) $x / D_{i}=6.5$

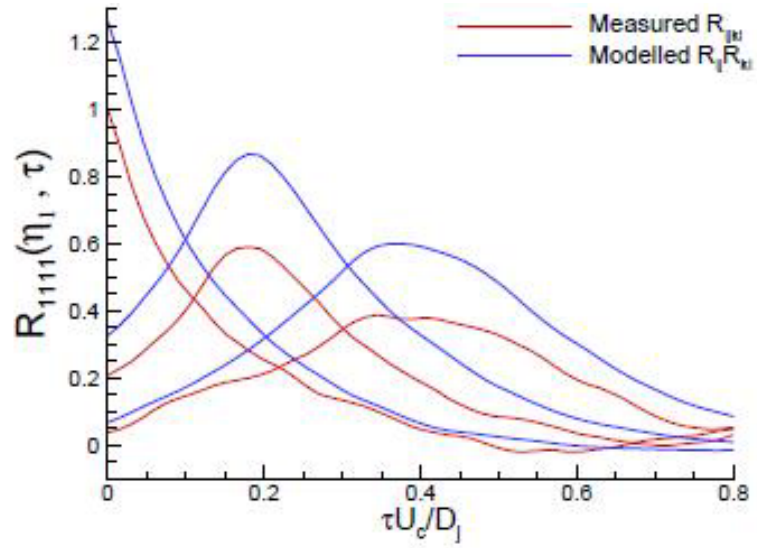

(b) $x / D_{j}=4$

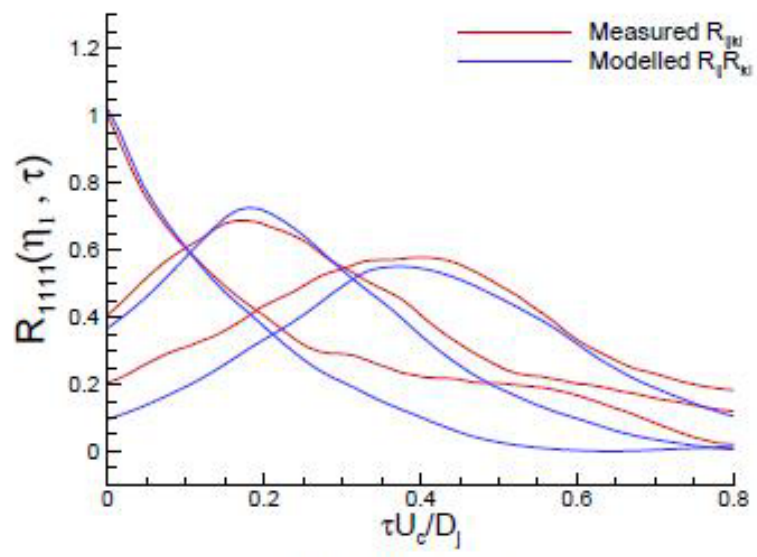

(d) $x / D_{i}=10$

FIGURE 29 (colour online) Comparison between measured and quasi-normal approximation of $R_{11,11}$

It is clear that it is not appropriate to focus attention just on $R_{11,11}$; the comparison of relative amplitudes shown in figure 27 illustrates the presence of several other important contributions. Figure 30 shows the comparison of directly measured and quasi-normal approximated quantities for the absolute amplitudes at zero spatial and temporal separations for the 6 largest components in the x-r plane (2C-PIV data are used here) at four shear layer locations. The previously observed over-estimation of the dominant $R_{11,11}$ component is seen again, but the agreement for the relative magnitude of the different correlation components is rated as good, providing direct evidence for the first time for multiple components on the accuracy of the quasi-normal approximation. In general the agreement is encouraging, but given the doubts expressed on the applicability of this approximation in shear flows (Morris and Zaman (2010)), it would be better if alternative, more generally valid approximations such as the generalised shell model recently proposed by Afsar (2012). 

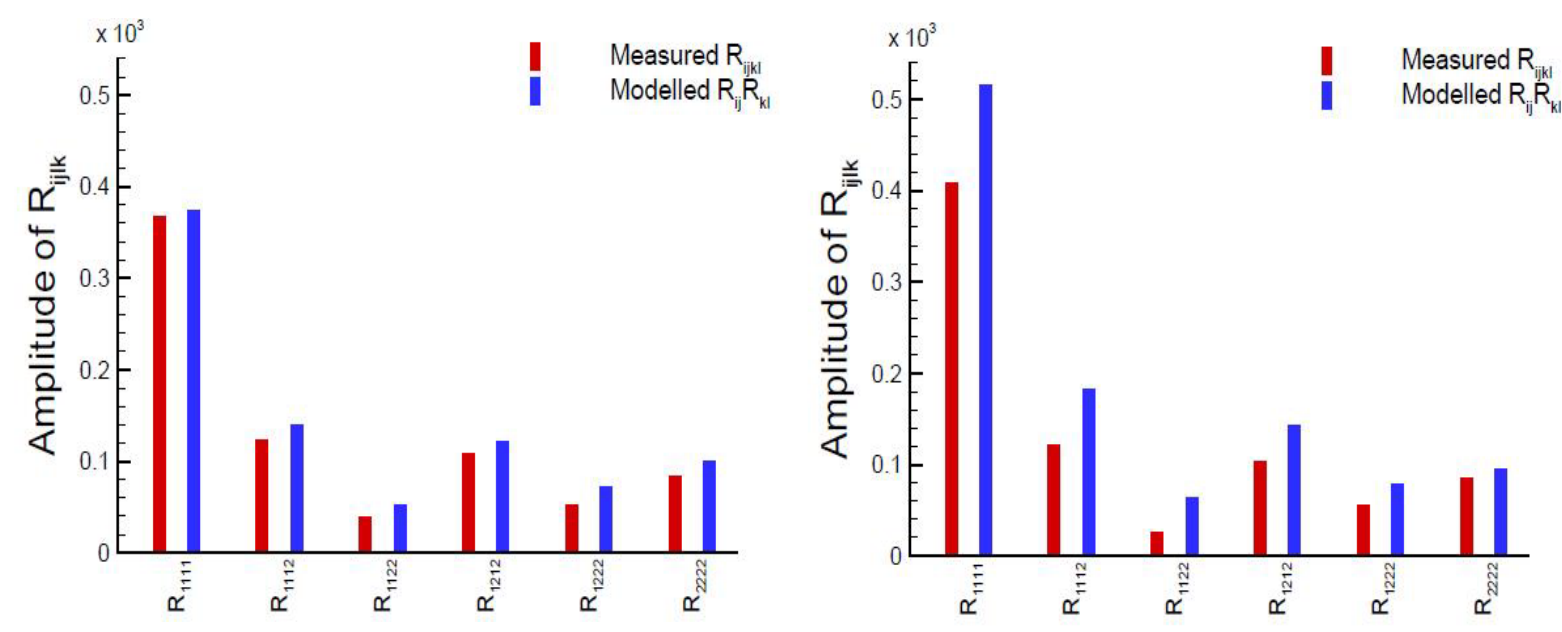

(a)

(b)
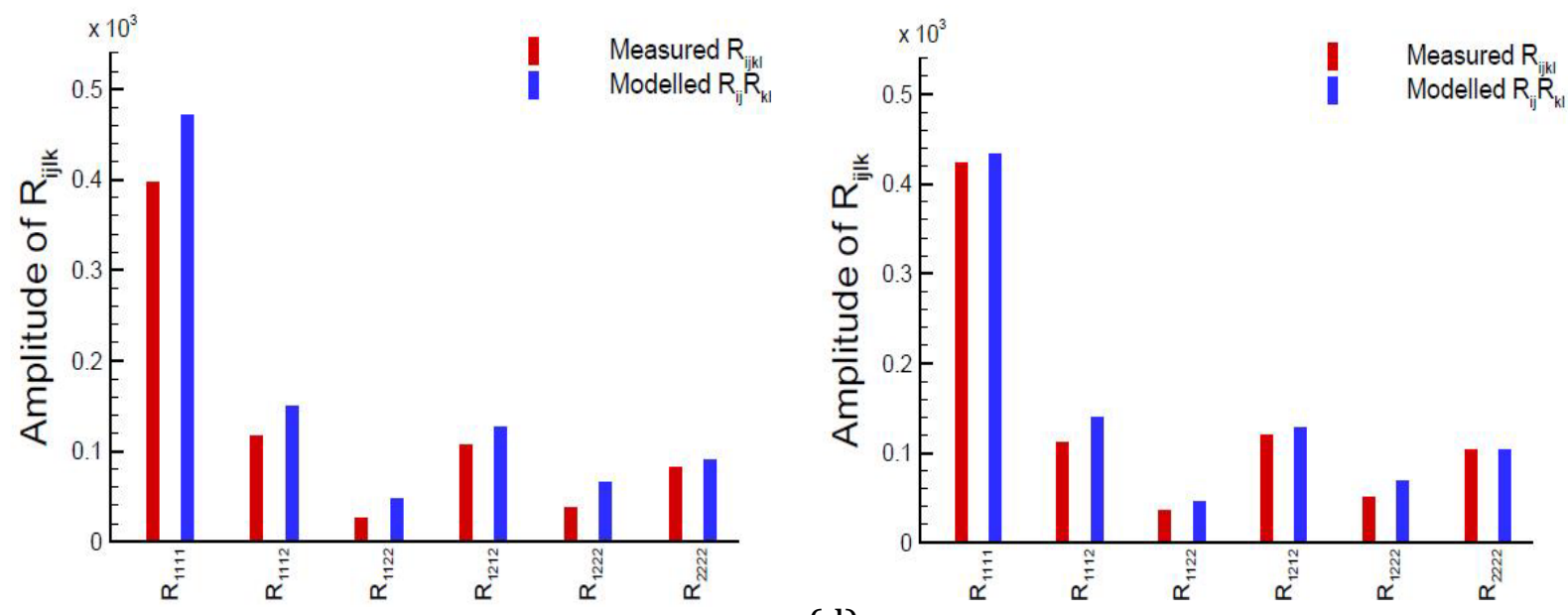

(c)

FIGURE 30 (colour online) Comparison between measured and quasi-normal approximation of $R_{i j, k l}$ in x-r plane shear layer: $\mathrm{x} / \mathrm{D}_{\mathrm{j}}=$ (a) 1.5 , (b) 4.0, (c) 6.5, (d) 10.0

\section{Concluding remarks}

The focus of the current work has been the need to obtain more detailed information about the $4^{\text {th }}$ - order 2-point 2-time spatio-temporal correlation $R_{i j, k l}$, which is the major source of turbulence generated noise in jets. Attempts to provide measurements of the many individual components of this in subsonic airflow experiments have proven problematic. An alternative approach has been followed in the present work, based on the assumption that the characteristics of this turbulence correlation are - at the high Reynolds numbers of practical relevance - not strongly affected by Reynolds number. In addition, for the subsonic Mach numbers of prime interest direct compressibility effects on the turbulence structure are minimal due to low convective Mach number. On this basis a water flow rather than an airflow experiment has been conducted, since the difference in kinematic viscosity between water and air means that the temporal scales of the turbulence which have to be resolved reduce by a factor of $\sim 15$, facilitating the measurements of many more components of $R_{i j, k l}$ than previously possible. This practice 
limits the applicability of the data set produced to subsonic isothermal jets; similar measurements in high speed and heated jets will require substantial further progress in the spatial/temporal resolution capabilities of PIV.

Stereoscopic PIV measurements were conducted in an axisymmetric turbulent jet within a water tunnel facility. Thorough comparison with existing data using both HWA and PIV in airflow experiments over a range of subsonic Mach numbers provided strong evidence that the measured turbulence structure was closely representative of high subsonic jet shear layers. An important aspect of the current work is the close attention paid to spatial filtering and the associated loss of measurement accuracy for turbulence components due to large PIV FoV size relative to local integral scales of turbulence. The correction methodology of Spencer and Hollis (2005) was adopted to identify appropriate PIV FoV sizes to avoid sub-cell turbulence errors, but also measure 2-point correlations over large separation distances. Comparison with single point data from other studies was shown to be good, with turbulent integral length scales estimated from the present water flow PIV measurements matching well those obtained from airflow experiments.

$2^{\text {nd }}$ and $4^{\text {th }}$ rank tensor spatio-temporal correlations have been evaluated from the present measurements and were shown to be of comparable accuracy to previous HWA results both near the nozzle exit (where the integral length scale is small) and further downstream. Implementation of a POD analysis and its use as a low pass filter was shown to be necessary when conducting 3C-PIV measurements. The POD-based correction method was demonstrated as a valid method for filtering 3C-PIV data containing high frequency noise. The low energy (i.e. high frequency) POD-based filter acted in a similar fashion to the anti-aliasing filter used for hot-wire probes, allowing for more accurate spectral data.

In addition to validating the stereoscopic 3C-PIV against HWA results (for the first time for multiple $4^{\text {th }}$-order components), whole-field visualisation, for all 3 velocity components, via spatio-temporal correlation maps proved possible. The data obtained are ideal for validating proposed models of $R_{i j, k l}$ for use in jet noise prediction. To date many of these models have made use of the quasi-normal approximation to relate $4^{\text {th-order }}$ correlations to $2^{\text {nd }}$-order; the present data showed this approach to be reasonable but also to contain several regions of discrepancy. The data presented could profitably be used to calibrate models of $R_{i j, k l}$ obtained from Large Eddy Simulation CFD as demonstrated by Karabasov et al. (2010) or models derived from theoretical considerations e.g. Afsar (2012).

\section{ACKNOWLEDGMENTS}

The authors gratefully acknowledge Marcus Harper-Bourne for his continual support with regards to access to raw data and interpretations of his HWA experimental study; also thanks to Mohammed Afsar for his input and helpful discussion on correlation definitions.

\section{REFERENCES}

AFSAR, M. Z. 2012 Insight into the two-source structure of the jet noise spectrum using a generalised shell model of turbulence European J. of Mech. B/Fluids 31, 129-139.

ALKISLAR, M. B. 2007 Correction of turbulence quantities in 3D PIV data. AIAA 2007-526, 45th AIAA Aerospace Science Conference. 
Alkislar, M. B., Krothapalli, A., \& Butler, G. W. 2007 The effect of streamwise vortices on the aeroacoustics of a Mach 0.9 jet. Journal of Fluid Mechanics 578, 139-169.

BATCHELOR G. K. 1953 The Theory of Homogeneous Turbulence Cambridge University Press, Cambridge, UK.

Behrouzi, P. \& McGuiRK, J. J. 2004 Jet mixing enhancement using fluid tabs. AIAA 20042401, 2nd AIAA Flow Control Conference.

Behrouzi, P. \& McGuirk, J. J. 2006 Effect of tab parameters on near-field jet plume development. AIAA Journal of Propulsion and Power 22, 576-583.

BRADSHAW, P., FERRIS, D. H., \& JOHNSON, R. F. 1964 Turbulence in the noise producing region of a circular jet. Journal of Fluid Mechanics 19, 591-621.

BRIDGES, J. \& WERNET, M. 2003 Measurements of the aeroacoustic sound source in hot jets.

AIAA 2003-3130, 9th AIAA/CEAS Aeroacoustics Conference.

BRIDGES, J. \& WERNET, M. 2010 Establishing consensus turbulence statistics for hot subsonic jets. AIAA 2010-3751, 16th AIAA/CEAS Aeroacoustics Conference.

BRIDGES, J. \& WERNET, M. 2012 Validating Large Eddy Simulation for jet aeroacoustics. AIAA Journal of Propulsion and Power 28, 226-234.

ChatellieR, L. \& FitzPATRicK, J. 2005 Spatio-temporal correlation analysis of turbulent flows using global and single-point measurements. Experiments in Fluids 38, 563-575.

Craya, A. \& CuRTet, R. 1955 On the spreading of a confined jet. C.R. Acad. Sci. Paris 241, 621-622.

DAVIES, P. O. A. L., FISHER, M. J., \& BARRATT, M. J. 1962 The characteristics of the turbulence in the mixing region of a round jet. Journal of Fluid Mechanics 15, 337-367.

Fellouah, H., BALl, C. G., \& Pollard, A. 2009 Reynolds number effects within the development region of a turbulent round free jet. International Journal of Heat and Mass Transfer 52, 3943-3954.

FISHER, M. J. \& DAVIES, P. O. A. L. 1964 Correlation measurements in a non-frozen pattern of turbulence. Journal of Fluid Mechanics 18, 97-116.

Fleury, V., BAilly, C., JondeAU, E., Michard, M., \& JuVE, D. 2008 Space-time correlations in two subsonic jets using dual-PIV measurements. AIAA Journal 46, 2498-2509.

HARPER-BOURNE, M. 1999 Jet near-field noise prediction. AIAA 1999-3214, $7^{\text {th }}$ AIAA/CEAS Aeroacoustics Conference.

HARPER-BOURne, M. 2003 Jet noise turbulence measurements. AIAA 2003-3214, 9th AIAA/CEAS Aeroacoustics Conference.

HoEst-MAdSEN, A. \& NiElSEN, A. H. 1995 Accuracy of PIV measurements in turbulent flows. ASME Laser Anemometry 229, 481-488.

Karabasov, S. A., Afsar, M. Z., Hynes, T. P., Dowling, A. P., McMullan, W. A., Pokora, C. D., PAGE, G. J. \& MCGUIRK, J. J. 2010 Jet noise; acoustic analogy informed by large eddy simulation. AIAA Journal 48, 1312-1325.

Kerherve, F., Jordan, P., Gervais, Y., Valiere, J. C., \& Braud, P 2004 Two-point laser Doppler velocimetry measurements in a Mach 1.2 cold supersonic jet for statistical aeroacoustic source model. Experiments in Fluids 37, 419-437.

Kerherve, F., FitZPATRICK, J., \& JoRdAN, P. 2006 The frequency dependence of jet turbulence for jet noise modelling. Journal of Sound and Vibration 296, 209-225.

LAU, J. C. 1980 Laser velocimeter correlation measurements in subsonic and supersonic jets. Journal of Sound and Vibration 70, 85-101.

LAU, J. C., MorRIS, P. J., \& FISHER M. 1979 Measurements in subsonic and supersonic free jets using a laser velocimeter. Journal of Fluid Mechanics 93, 1-27.

LAURENCE, J. C. 1956 Intensity, scale, and spectra of turbulence in the mixing region of a free subsonic jet. NACA Report No. 1292. 
LEIB, S. J. \& GolDSTEIN, M. E. 2011 Hybrid source model for predicting high speed jet noise. AIAA Journal 49, 1324-1335.

LIEPMANN, H., W., LAUFER, J. 1947 Investigations of free turbulent mixing NACA TN-1257.

Lighthill, M. J. 1952 On sound generated aerodynamically - i: general theory. Proc. R. Soc. Lond. 211, 564-587.

LIGHTHILL, M. J. 1954 On sound generated aerodynamically - ii: turbulence as a source of sound. Proc. R. Soc. Lond. 222, 1-32.

LILLEY, G. M. 1958 On the noise from air jets. Aero. Res. Council (London) 20, 20-27.

MiDGLEY, K., SPENCER, A., and MCGUIRK, J. J. 2005 Unsteady flow structures in radial swirler fed fuel injectors. ASME Journal of Eng. for Gas Turbines and Power 127, 755-764. MillionshCHIKOV, M. D. 1941 On the theory of homogeneous isotropic turbulence. Dokl. Akad. Nauk SSSR 32, 611-614.

MoRRIS, P. J. \& FARASSAT, F. 2002 Acoustic analogy and alternate theories for jet noise prediction. AIAA Journal 40, 671-680.

MoRRIS, P. J. \& ZAMAN, K. B. M. Q. 2010 Velocity measurements in jets with application to noise source modelling. Journal of Sound and Vibration 329, 394-414.

PAPAmoschou, D. \& RoshKo, A. 1988 The compressible turbulent shear layer: an experimental study. Journal of Fluid Mechanics 197, 453-477.

Proudman, I.1952 The generation of noise by isotropic turbulence. Proc. R. Soc. Lond. 214,119-132

PoKoRA, C. D. 2009 Spatio-temporal correlations of jets using high speed particle image velocimetry. PhD thesis Loughborough University.

PoKoRA, C. D. \& McGuiRK, J. J. 2008 Spatio-temporal correlations using high-speed PIV in an axisymmetric jet. AIAA 2008-3028 14th AIAA/CEAS Aeroacoustic Conference.

Power, O., Kerherve, F., FitzPatrick, J., \& Jordan, P. 2004 Measurements of turbulence Conference.

RicoU, F. \& SPALDinG, D. B. 1961 Measurements of entrainment by axisymmetric turbulent jets. Journal of Fluid Mechanics 11, 21-32.

SELF, R. H. 2004 Jet noise prediction using the Lighthill acoustic analogy. Journal of Sound and Vibration, 275, 755-768.

SPENCER, A. \& HolLIS, D. 2005 Correcting for sub-grid filtering effects in particle image velocimetry data. Measurement Science and Technology 16, 2323-2335.

WeitaO, B., YASUHIKo, S., \& HARUKI, M. 2003 Time-resolved proper orthogonal decomposition of the near-field flow of a round jet measured by dynamic particle image velocimetry. Measurement Science \& Technology 14, L1-L5.

WERNET, M. P. 2007 Temporally-resolved PIV for space-time correlations in both cold and hot jet flows. Measurement Science and Technology 18, 1387-1403.

WesterweEl, J., DraAd, A. A., VAN DER HoEven, J. G. T., \& VAN OORD, J. 1996 Measurement of fully-developed turbulent pipe flow with digital particle image velocimetry. Experiments in Fluids 20, 165-177. 